\title{
Structural and Functional Insights into Interactions of Oligomeric $\alpha$-Synuclein with Lipid Membranes
}

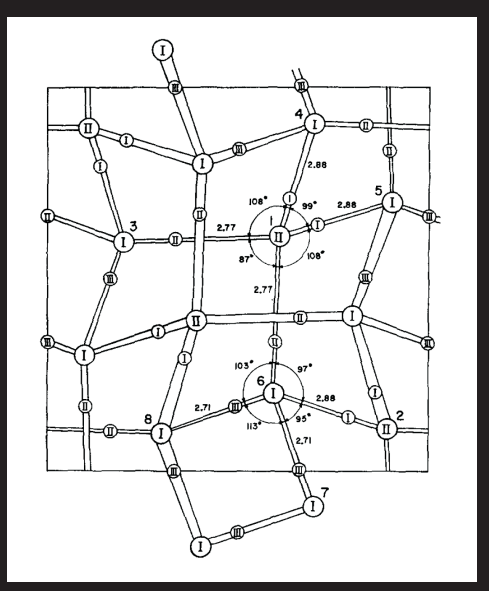

B.D. van Rooijen 
STRUCTURAL AND FUNCTIONAL INSIGHTS INTO INTERACTIONS OF OLIGOMERIC $\alpha$-SYNUCLEIN WITH LIPID MEMBRANES

B.D. van Rooijen 
Thesis committee members:

$\begin{array}{lll}\text { Prof. dr. } & \text { G. van der Steenhoven } & \text { Universiteit Twente (chairman) } \\ \text { Prof. dr. } & \text { V. Subramaniam } & \text { Universiteit Twente (thesis advisor) } \\ \text { Dr. } & \text { M.M.A.E. Claessens } & \text { Universiteit Twente (assistant advisor) } \\ \text { Prof. dr. } & \text { D.E. Otzen } & \text { Aarhus University } \\ \text { Prof. dr. } & \text { J.A. Killian } & \text { Universiteit Utrecht } \\ \text { Prof. dr. } & \text { P. Heutink } & \text { VU medisch centrum } \\ \text { Prof. dr. } & \text { J.J.L.M. Cornelissen } & \text { Universiteit Twente } \\ \text { Prof. dr. } & \text { W.J. Briels } & \text { Universiteit Twente }\end{array}$

Paranimfen:

Dr. Ir. P. Zijlstra

M. Sc. N. Zijlstra

This work was part of the Nanotechnology network in The Netherlands (Nanoned), project number 7921 within the Nanoned programme Bionanosystems.

The work described in this thesis was performed at the Biophysical Engineering Group, MESA+ Institute for Nanotechnology, Faculty of Science and Technology, University of Twente, PO Box 217 7500AE Enschede, The Netherlands.

Copyright (C) 2009, B.D. van Rooijen, All rights reserved.

This thesis can be downloaded from http://dx.doi.org/10.3990/1.9789036529310.

Cover image reprinted with permission from: "Ice IX: An Antiferroelectric Phase Related to Ice III", E. Whalley et al., Journal of Chemical Physics (1968). Copyright 1968, American Institute of Physics. The image before the Table of Contents page was reprinted with permission from the AAAS from "The Structure of Polywater", J. Donohue, Science (1969).

Printed by Wöhrmann Print Service

ISBN 978-90-365-2931-0 


\title{
PROEFSCHRIFT
}

\author{
ter verkrijging van \\ de graad van doctor aan de Universiteit Twente, \\ op gezag van de rector magnificus, \\ prof. dr. H. Brinksma, \\ volgens besluit van het College voor Promoties \\ in het openbaar te verdedigen \\ op donderdag 26 november 2009 om 16.45 uur
}

door

Bart Dirk van Rooijen geboren op 8 maart 1981

te Warmenhuizen 
Dit proefschrift is goedgekeurd door:

Prof. dr. V. Subramaniam (promotor) en

Dr. M.M.A.E Claessens (assistent-promotor) 


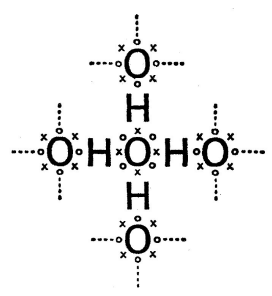

...its power is only exceeded by its mystery

- Dude where's my car? (2000) 



\section{Table of Contents}

Chapter 1 Introduction 1

Chapter $2 \quad$ Oligomeric $\alpha$-synuclein and its role in 29 aggregation

Chapter 3 Lipid bilayer disruption by oligomeric $\alpha$-synuclein

Chapter $4 \quad$ Membrane binding of oligomeric $\alpha$-synuclein

Chapter 5 Quantifying oligomer-lipid binding using fluorescence correlation spectroscopy

Chapter 6 Tryptophan fluorescence reveals structural features of $\alpha$-synuclein oligomers

Chapter $7 \quad$ Membrane permeabilization by oligomeric $\alpha$-synuclein: In search of the mechanism

Chapter 8 Conclusions, summary and outlook 



\section{Chapter 1 Introduction}

Parkinson's disease (PD) is a common neurodegenerative disorder associated with old age (1). Currently the disease cannot be cured, and treatment focuses on alleviating the symptoms of the disease (2). A major challenge in the development of an effective treatment is that in the majority of cases it is not known what causes the disease. The neuronal protein $\alpha$-synuclein $(\alpha \mathrm{S})$, is thought to play a pivotal role in the onset and progression of Parkinson's disease. It is the major constituent of the Lewy body and Lewy neurites, the intracellular inclusion bodies that are the neuropathological hallmark of the disease $(3,4)$. Furthermore, three point mutations in the gene encoding for $\alpha \mathrm{S}$, as well as gene duplications and triplications lead to rare familial forms of $\mathrm{PD}$ (5-9). Since Lewy bodies contain $\alpha \mathrm{S}$ in an aggregated filamentous form, the misfolding and aggregation of the protein into insoluble inclusion bodies are thought to contribute to neurotoxicity. However, a causal relation has never been established $(10,11)$. In recent years the attention has shifted to the early stages of the aggregation process. Early oligomeric intermediates in the aggregation of $\alpha \mathrm{S}$ have been found to be more toxic to cells compared to the monomeric or fibrillar forms of the protein $(12,13)$. A possible mechanism of neurotoxicity is disruption and permeabilization of cellular membranes by these soluble oligomers (14-16). Oligomeric $\alpha \mathrm{S}$ has been shown to induce increased membrane permeability in cultured cells and synthetic lipid bilayer systems (12, 16). However, very little is known on what causes these oligomers to interact with membranes, and how they permeabilize the membrane. In this work we have focused on elucidating how oligomeric $\alpha \mathrm{S}$ interacts with lipid bilayers. Using a strictly in vitro approach we have used a wide range of biophysical techniques to approach this problem. In this chapter the lines of evidence that have defined our initial questions will be described and the concepts necessary to understand this work will be introduced. Furthermore, the existing literature on the role of $\alpha \mathrm{S}$ in $\mathrm{PD}$ will be summarized in order to place the work in the broader context of the research field. 


\section{Protein misfolding and disease}

The genetic code determines the amino acid sequence of a protein. However, for most proteins to function correctly, they have to be folded in a specific conformation. Protein folding is a complex process, and the number of possible conformations for a polypeptide is astronomically large. Especially for larger proteins, folding rates can be extremely slow $(17,18)$. During folding the protein stochastically samples different conformations. Thermodynamically favorable interactions will increase the stability of partially folded intermediates, and during the folding process many intermediate states are sampled. This can be intuitively described by regarding the possible conformations and interactions as an energy landscape for the protein. Folding is energetically favorable and follows a downhill trajectory until it reaches the most thermodynamically stable fold. Generally this is also the conformation in which the protein needs to be folded in order to function and is often referred to as the native state (19, $20)$.

The process of folding is prone to errors and proteins can get "trapped" in the energy landscape in a non-native, "misfolded" conformation $(18,20)$. Local minima in the energy landscape lead to partially stable folding intermediates. Protein misfolding can thus directly occur when the protein is newly synthesized. In addition, the native state is often only marginally stable (21). Correctly folded proteins are therefore still in equilibrium with partially folded states and can also convert to a misfolded state. Possibly, there is a tradeoff between protein stability and function, and some conformational flexibility is required to allow the protein to interact with other components such as other proteins, DNA or membranes $(22,23)$. Another process that leads to misfolded protein is through chemical modification. Proteins in the cell are under constant attack of reactive molecules which can stabilize a non-native state (24). To remain functional, the cell thus has to actively maintain a pool of correctly folded and functional protein and has to deal with misfolded proteins in order to prevent the accumulation of misfolded dysfunctional protein.

Multiple pathways are present in cells to keep the protein pool under control. Chaperones and heat shock proteins assist newly synthesized proteins in folding correctly and can rescue misfolded proteins and refold them into the native state (25). If the correct fold cannot be achieved, misfolded proteins are targeted to the cellular degradation machinery, such as the ubiquitin proteasome system $(24,26,27)$. The protein quality control and degradation pathways can be severely challenged by misfolded proteins. Cell stress, for instance by exposure to pathogens, puts a high demand on protein expression and folding pathways. Genetic mutations that adversely affect the stability of the native state of a protein also increase the amount of misfolded protein the cell has to cope with 
(28). Finally, it has been recognized that the ability to maintain a healthy cellular protein housekeeping (proteostasis) declines with age (29).

If the capacity of an organism to deal with misfolded proteins is impaired, this often leads to disease $(20,28,30)$. This can be either through a loss of functional protein or through a gain of toxic function of the misfolded protein. Misfolded proteins are generally much more aggregation prone compared to the native state. Therefore, protein misfolding diseases are often accompanied by protein aggregation (20). The list of diseases in which protein misfolding and aggregation appear crucial is expanding rapidly and contains a wide range of conditions. In systemic amyloidoses circulating proteins can accumulate in extremely large amounts (sometimes several kilograms) in multiple organs (31). Often protein aggregation is more local and occurs in the organ where the protein is expressed. In type II diabetes, aggregates of the protein amylin are found in the pancreas (32). Perhaps the most notorious member of protein aggregation diseases is Creutzfeldt Jakob's disease, in which neurodegeneration is accompanied by misfolding and aggregation of prion protein (33). If misfolding of the prion protein occurs, the misfolded species can subsequently convert normally folded conformers to the misfolded state (34). Prion diseases can thus be infectious; contact with misfolded protein can propagate the misfolding of the protein. The disease might even cross the species barrier such that contact with prions from bovine spongiform encephalopathy (mad cow disease) can lead to a human Creutzfeldt Jakob variant disease.

Remarkably, many of the protein aggregates found in the different diseases share a similar structure, even though the sequence, structure and function of the original proteins are very different (35). This common structure is referred to as "amyloid". Although there does not exist a clear definition of the term amyloid, the following properties are generally considered characteristic of amyloid structure: Aggregates have a fibrillar unbranched morphology. They bind to the dyes Congo red, showing apple green birefringence, and thioflavin- $T$, which increases its fluorescence quantum yield upon binding $(20,36)$. Structurally, the fibrils consist of stacked cross $\beta$-sheets, in which strands run perpendicular to the fibril axis and intermolecular hydrogen bonding between the $\beta$-sheets occurs along the fiber axis $(37,38)$. Since the amyloid fibrils are stabilized by multiple hydrogen bonds, they are extremely stable and are neither easily dissolved in denaturants like SDS and Sarkosyl nor easily digested by proteases $(39,40)$. The remarkable stability of the amyloid fold might make it difficult for the cell to get rid of these aggregates, which is a possible reason why they are observed in so many diseases. However, it is still not clear if protein aggregation reports on the fact that cellular proteostasis is disturbed, or if aggregation is actively involved in disturbing normal cell processes (28). 


\section{Parkinson's disease}

Parkinson's disease (PD) is a progressive neurodegenerative disorder with a late average age of onset (1). Although cognitive disturbances are very common, it is primarily known as a movement disorder. The disease is characterized by several motor symptoms, such as tremor, limp stiffness, slowness of movement and impaired posture and balance. The most common pathophysiological definition of the disease is the loss of dopaminergic neurons in the substantia nigra, a region in the brain which is important for movement $(41,42)$. The cell loss is accompanied by the occurrence of Lewy bodies and Lewy neurites, which are intracellular inclusion bodies composed of aggregated protein $(3,41)$. Diagnosing $\mathrm{PD}$ is not straightforward; motor scoring tests are prevalent but are not considered conclusive. Final diagnosis is generally established post-mortem on the basis of Lewy body pathology $(2,42)$. There is probably not a single well defined disorder which can be called PD. A range of disorders are known that are characterized by Lewy body pathology and neurodegeneration (42). Currently $\mathrm{PD}$ cannot be cured and treatment is directed at alleviating the disease symptoms (2). One of the main challenges in the development of a cure for PD is that the actual cause of the disease is not known. In a very small number of cases there is a clear genetic cause. Several mutations in a number of genes have been identified as either a cause or a risk factor for PD $(43,44)$. In addition, environmental factors may contribute to disease onset. For instance, long term occupational exposure to pesticides and heavy metals has been reported to increase the risk of PD $(45,46)$. However, the vast majority of PD cases are idiopathic and in fact the most well established risk factor for PD is old age.

\section{$\alpha$-Synuclein and Parkinson's disease}

One of the possible key players in the onset and progression of PD is the neuronal protein $\alpha \mathrm{S}$. Interest in this protein was raised when it was discovered as the main component of Lewy bodies, the neuropathological hallmark of PD (4). The discovery that a point mutation in the gene coding for $\alpha \mathrm{S}$ causes a rare familial form of $\mathrm{PD}$ confirmed the importance of this protein to the disease (7). Since then, two additional mutations in the $\alpha \mathrm{S}$ gene have been identified that lead to a genetic form of $\mathrm{PD}(6,9)$. Additionally, $\alpha \mathrm{S}$ gene duplication and triplication have also been found to cause PD $(5,8)$. Genetic evidence thus suggests that both mutant $\alpha \mathrm{S}$ and higher levels of $\alpha \mathrm{S}$ can be causative for familial PD. However, a causal relation between $\alpha \mathrm{S}$ and idiopathic PD has never been established. Additional evidence supporting the role of $\alpha \mathrm{S}$ as a possible causative factor for neuronal degeneration comes from transgenic animal models. Especially rodent models have been extensively used to try to replicate the 
characteristics of PD (47) but also fruit fly (48) and worm models have been developed (49). Many of these models indeed show neurodegeneration upon expression of $\alpha \mathrm{S}$. However the characteristics of the different models vary, with for instance the promoter used for $\alpha \mathrm{S}$ expression (50). Therefore, extracting meaningful mechanistic information from these models still poses a challenge.

Since Lewy body pathology is considered the neuropathological hallmark of $\mathrm{PD}$, the relation between the occurrence of Lewy bodies and disease progression could hold valuable information about the role of $\alpha \mathrm{S}$ in PD. Thorough investigation of the distribution of Lewy bodies in post mortem brain samples of PD patients has led to the hypothesis that PD pathology follows a distinct route and that the distribution of Lewy bodies might correlate with the clinical stage of PD (41). However, it has also been reported that in a number of cases the occurrence of Lewy bodies does not correlate with the clinical severity of the disease. For instance, Lewy bodies have been found in brain samples from patients that did not display any symptoms of PD $(51,52)$. In addition, it has never been shown that $\alpha \mathrm{S}$ inclusion body formation is a neurotoxic event (53, $54)$. Thus, a key question remains if Lewy bodies are merely a sign of neuronal stress or the actual cause of neuronal toxicity.

\section{Properties of $\alpha$-synuclein}

Human $\alpha \mathrm{S}$ is a 140 amino acid (14 kDa) protein which is abundantly expressed throughout the central nervous system $(55,56)$. It is mainly localized near the presynaptic terminal of neuronal cells (57-59), but $\alpha \mathrm{S}$ has also been observed in the cytosol $(59)$ and nucleus $(60,61)$. At physiological $\mathrm{pH}$, the protein has no defined secondary structure and is therefore considered an intrinsically disordered protein (IDP) (62). Residues 7-87 contain six imperfect repeats of an amino acid sequence (KTKEGV consensus sequence), and have a propensity to assume an $\alpha$-helical conformation $(63,64)$. The central region (residues 61 $95)$ is very hydrophobic and is crucial in the aggregation of $\alpha \mathrm{S}(40,65)$. The C-terminus (residues $\sim 100-140$ ) of the protein is highly negatively charged and remains largely unstructured under most conditions and may serve as a solubilizing domain. The calculated net charge at neutral $\mathrm{pH}$ for residues $100-$ 140 is approximately -13 , while residues 1-100 have a net charge of around +4 . The disease related point mutations (A30P, E46K and A53T) are all located in the N-terminal part of the protein. Most of the post translational modifications of $\alpha \mathrm{S}$ are observed in the C-terminus of the protein. Serine 129 has been reported as the major site for phosphorylation (66) while tyrosines 125, 133 and 136 are vulnerable to nitration (67). These $\alpha \mathrm{S}$ modifications are also commonly observed inside Lewy bodies. Additionally, C-terminally truncated species $\alpha \mathrm{S} 112$ and 126 are also present inside Lewy bodies $(68,69)$. 
Most of these modifications have an impact on the biochemical properties of $\alpha \mathrm{S}$, such as aggregation propensity $(70,71)$. They are also likely to be important in modulating the biological function of the protein $(72,73)$.

A complicating factor in determining the role of $\alpha \mathrm{S}$ in $\mathrm{PD}$ is that its normal biological function is not understood (74). This might be related to the fact that $\alpha \mathrm{S}$ is an IDP $(62,75)$. A notable feature of IDPs is that they escape the normal structure-function paradigm of proteins. They are considered to be much more dynamic and to modulate their function by changing their conformation. Possibly, this allows IDPs to interact with a large number of proteins and macromolecules (76).

It has been suggested that $\alpha \mathrm{S}$ plays a role in neurotransmission (55). Although transgenic $\alpha \mathrm{S}$ knockout mice are viable and fertile, changes in behavior, dependent on the dopaminergic neurons have been observed $(77,78)$. In addition, alterations in the vesicle reserve pool in these mice have been observed by electron microscopy on extracted brain tissue (79). A role in modulation of neurotransmission is further supported by several studies that indicate $\alpha \mathrm{S}$ inhibits the dopamine transporter $(80,81)$. Additionally, $\alpha \mathrm{S}$ has been shown to inhibit phospholipase-D2 which is important for membrane trafficking fusion/fission through the generation of the bioactive phospholipid, phosphatidic acid (82). In vitro it has been shown that $\alpha \mathrm{S}$ can bind to phospholipid membranes (63). Binding is mediated by residues $\sim 1-100$ which fold into an amphipathic $\alpha$-helix upon membrane binding $(64,83,84)$. Together these results implicate that $\alpha \mathrm{S}$ may be important in maintaining a functional synapse. Interestingly, it has also been suggested that $\alpha \mathrm{S}$ can have a neuroprotective role under some conditions. $\alpha \mathrm{S}$ overexpression in cell and animal models has been reported to be neuroprotective against oxidative stress $(85,86)$, exposure to pesticides (87) and other types of insult $(88,89)$. In addition, it has been hypothesized that $\alpha \mathrm{S}$ could possess a chaperone like activity. Structurally and functionally $\alpha \mathrm{S}$ possesses similarities to chaperone proteins (90-92). This activity is most likely mediated by the negatively charged and unstructured C-terminus of the protein $(93,94)$.

\section{Possible disease mechanisms}

Although PD has been the subject of extensive research for many years, little is known about the actual mechanism of neuronal cell death. Several contributing mechanisms are often mentioned in the literature. Although they are all supported by a reasonable body of evidence, the true relevance of these mechanisms to PD is not established and can only be speculated on. A short description of some of these possible disease mechanisms is written below to give the reader an insight in the complexity of the disease. 
Introduction

\section{Oxidative stress}

Reactive oxygen species are continuously generated by the cell's metabolic processes. In order to function properly, the cell has to prevent the accumulation of these species and actively maintain its redox potential. When this ability is compromised, an excess of reactive oxygen species poses a toxic challenge to the cell (95). There is convincing evidence that oxidative stress is present in the brain regions affected in PD. Lipid peroxidation, DNA/RNA damage and protein oxidation have been observed to be elevated in PD patients $(67,96)$. In addition, a genetic variant of PD is caused by a mutation of the protein DJ-1, which is thought to be involved in the cell's response to oxidative stress (97). Several processes, such as exposure to metals and toxins (98), dopamine synthesis (96) and mitochondrial dysfunction (99), could to lead to an increased oxidative stress in the brain and thus play a role in the disease. It still remains unclear what causes these signs of oxidative stress in the PD brain, and if this is a prime cause of $\mathrm{PD}$ or a late step contributing factor. Attempts to slow down the progression of PD by the admission of antioxidants have however not proven to be an effective treatment for the disease (2).

\section{Dopamine}

The neurotransmitter dopamine has long been hypothesized to contribute to PD (100). One of the main reasons to consider dopamine as a key player in toxicity is that the dopaminergic neurons in the substantia nigra are considered to be affected most in PD. Non-dopaminergic neurons are also affected, although to a lesser extent (42). Dopamine metabolites are neurotoxic and can induce reactive oxygen species $(96,101)$. Interestingly, dopamine and its metabolites are known to directly influence $\alpha \mathrm{S}$ aggregation (102). Furthermore, $\alpha \mathrm{S}$ potentially influences dopamine homeostasis through interaction with the dopamine transporter which transports free dopamine from the synapse into the cell (81, 103) and with tyrosine hydroxylase, an enzyme involved in dopamine synthesis (104).

\section{Mitochondrial dysfunction}

Mitochondria have been thought to play an important role in PD for many years. Mitochondria are the cell's main source of reactive oxygen species and are also important in regulating apoptotic cell death. PD patients have been reported to show a reduced mitochondrial complex I activity in the brain and skeletal muscle (105-107), a protein complex vital in the mitochondrial respiratory chain. Several mitochondrial toxins such as MPTP (1-methyl-4-phenyl1,2,3,6-tetrahydropyridine) can reproduce PD-like symptoms and neurodegen- 
eration through complex I inhibition. In addition, several genes that have been associated with genetic variants of PD encode proteins have been linked to mitochondrial function (PINK1, parkin, DJ-1) (108). In animal models, overexpression of $\alpha \mathrm{S}$ induces mitochondrial abnormalities $(109,110)$. Although it is not clear how $\alpha \mathrm{S}$ can influence mitochondrial function, $\alpha \mathrm{S}$ shows a mitochondrial localization under specific conditions $(111,112)$.

\section{Neuronal inflammation}

There is clear evidence that the affected brain regions in PD are characterized by an inflammatory response. Activated microglia $(113,114)$ and higher levels of pro-inflammatory cytokines have been reproducibly found in post-mortem studies on brain samples (115). Inflammatory responses can exert toxic effects through the production of reactive oxygen species and through the activation of apoptotic pathways by cytokines. The inflammatory response could spread through the brain and thus cause the progressive nature of the disease. A decreased risk for PD has been reported in people that take anti-inflammatory drugs $(116,117)$, which indicates that inflammatory responses might indeed contribute to the onset or progression of $\mathrm{PD} . \alpha \mathrm{S}$ has been reported to interfere with inflammatory pathways. Cell culture studies have indicated that cytokines affect the cellular distribution of $\alpha \mathrm{S}$ (118). Furthermore, both monomeric and aggregated $\alpha \mathrm{S}$ have been observed to activate microglia $(119,120)$.

\section{Proteasomal inhibition}

The ubiquitin-proteasome system (UBS) is an important protein degradation mechanism for the cell, and is therefore of prime interest in many protein aggregation diseases (121). Dysfunction of the UBS might also play an important role in PD. Lewy bodies contain considerable amounts of ubiquitinated proteins $(122,123)$. In addition, there are indications for reduced proteasomal activity in the substantia nigra of PD patients (124). Genetic evidence also points to a potential role of the UBS in PD. Mutations in the proteins parkin (125) and UCHL-1 (126) that are involved in the UBS have been reported to be causative of $\mathrm{PD}$. How $\alpha \mathrm{S}$ relates to the UBS has been intensely investigated in recent years. Inhibition of UBS function induces $\alpha \mathrm{S}$ aggregation, inclusion body formation and toxicity in both cell and animal models $(127,128)$. However the exact mechanism by which this occurs remains unclear, since the UBS might not be necessary for $\alpha \mathrm{S}$ degradation. The autophagy-lysosomal pathway has also been reported to be an important degradation pathway for $\alpha \mathrm{S}$ (129-132). Interactions between $\alpha \mathrm{S}$ and the UBS might also have deleterious effects for the cell. Proteasomal inhibition by monomeric as well as oligomeric and fibrillar $\alpha \mathrm{S}$ have been reported (133-135). These data suggest a possible positive feedback 
Introduction

loop where $\alpha \mathrm{S}$ aggregation inhibits the proteasome which decreases the cell's ability to deal with aggregated protein which then leads to increased $\alpha \mathrm{S}$ aggregation. In addition, proteasome activity is known to decline with increasing age (29).

\section{$\alpha$-Synuclein and the amyloid pore hypothesis}

The possible disease mechanisms that are mentioned in the previous section are all promising lines of research that are being pursued by the many groups working in this field. Given the techniques we have available in our lab we have chosen to focus on another potential disease mechanism called the "amyloid pore hypothesis". The reasoning behind this hypothesis will be introduced below.

In many of the amyloid forming proteins that are related to human diseases, the consensus is that a gain of toxic function of the protein aggregates contributes to the disease (136). However, many of the features of these diseases cannot be explained from the viewpoint that the final protein aggregates are toxic to cells $(137,138)$. This observation was first noted for Alzheimer's disease, in which the protein $\mathrm{A} \beta$ aggregates to form extracellular plagues. A lack of correlation between the amounts of aggregates found on autopsy and the clinical severity of the disease was reported (139). Additionally, animal models overexpressing $\mathrm{A} \beta$ revealed that the characteristic symptoms of the disease preceded the plaque formation $(140,141)$. This prompted the hypothesis that perhaps earlier intermediates, called "oligomers", in the aggregation process might be the toxic species responsible for neurodegeneration (142). For A $\beta$ there is indeed a growing body of evidence that oligomers are much more toxic to cells than monomeric or fibrillar species (138).

It was soon recognized that similar to amyloid fibrils, oligomers from different amyloidogenic proteins share common structural and functional characteristics (13). It has been found for a number of amyloid proteins and even for non-disease related aggregating protein such as the bacterial protein Hypf-N, that oligomeric intermediates are toxic to cells (13, 143-145). Furthermore, antibodies have been raised that specifically recognize oligomers from different amyloid proteins, suggesting they share a common fold $(13,146)$. Amyloid oligomers also show morphological similarities. They are often reported as spherical protein aggregates that show an annular morphology with sizes ranging from 8-12 nm (144). It was thus postulated that since amyloid oligomers appear to have similar structures they may also have a common mechanism of toxicity (13).

The "amyloid pore hypothesis" proposes that the common toxic mechanism of amyloid oligomers is disruption of calcium homeostasis through per- 
meabilization of lipid membranes $(14,147)$. This hypothesis originated from Alzheimer's disease research in which it was long recognized that disruption of calcium homeostasis could contribute to the disease, and that $\mathrm{A} \beta$ could show channel-like activities in lipid bilayers (148). The role of oligomeric $\alpha \mathrm{S}$ in PD is not well established $(11,137)$. In parallel to Alzheimer's disease it is often argued that $\alpha \mathrm{S}$ oligomers are more likely to be toxic than $\alpha \mathrm{S}$ fibrils. In PD the occurrence of Lewy bodies does not unambiguously correlate with disease progression and severity $(51,52)$. Lewy body pathology has been observed on autopsy of individuals that did not show any symptoms of PD (52). The mere fact that Lewy bodies are observed intracellularly in intact neurons may already indicate that they are at least not directly toxic to the cell. Numerous mice and Drosophila models of PD that rely on $\alpha \mathrm{S}$ overexpression have been generated. PD like symptoms are reproduced but Lewy body-like pathology and protein aggregation is not always observed (149-151). The disease related mutants of $\alpha \mathrm{S}$ form oligomers more readily than the wild-type (wt) protein, but the A30P mutant forms fibrils more slowly than $\alpha \mathrm{S}$-wt (152). Finally, when added extracellularly to cultured cells, oligomeric $\alpha \mathrm{S}$ is more toxic than the monomeric and fibrillar forms of the protein $(12,146,153)$.

These findings imply that oligomeric $\alpha \mathrm{S}$ rather than fibrillar $\alpha \mathrm{S}$ is responsible for cytotoxicity in PD. Similar to other amyloid oligomeric intermediates, it was subsequently investigated if membrane disruption by $\alpha \mathrm{S}$ oligomeric could be a possible cytotoxic mechanism. A small body of evidence suggests that membrane disruption by oligomeric $\alpha \mathrm{S}$ is indeed a possible mechanism of toxicity. Annular pore-like morphologies have been observed using in vitro generated oligomers $(144,154,155)$ but also in tissue derived $\alpha \mathrm{S}$ preparations (156). Oligomers have been reported to disrupt phospholipid vesicles possibly through a pore-like mechanism, based on a size selectivity of marker efflux (157). When reconstituted in planar lipid bilayers, increased ion conductivity has been reported (144), either with distinct pore-like current jumps or through a decrease in membrane thickness (14). When added to cultured cells, $\alpha \mathrm{S}$ oligomers have been shown to increase calcium influx (12). More recently, it has also been suggested both through simulations and conductivity measurements on planar bilayers that monomeric $\alpha \mathrm{S}$ can aggregate on the membrane and directly form small pore-like structures $(158,159)$.

Given the limited amount of experimental evidence, the topics of membrane disruption by $\alpha \mathrm{S}$ and the role of oligomeric $\alpha \mathrm{S}$ species in $\mathrm{PD}$ are still a matter of intense debate and there are still many open questions. How $\alpha \mathrm{S}$ oligomer membrane interaction looks like on a molecular scale is completely unknown. For instance the actual mechanism of membrane disruption and the structural characteristics of $\alpha \mathrm{S}$ oligomers still remain to be determined. 


\section{Scope of this thesis}

The membrane interaction of oligomeric $\alpha \mathrm{S}$ is often regarded as a potential mechanism of neurotoxicity in PD. However, surprisingly little is actually known about this interaction and membrane disruption by $\alpha \mathrm{S}$ is still a recurring topic of speculation and debate. In this work we aim to investigate the interaction between oligomeric $\alpha \mathrm{S}$ and lipid bilayers. The following fundamental research questions have guided our experimental work:

- What physical membrane properties modulate the interaction between $\alpha \mathrm{S}$ oligomers and the membrane?

- What structural properties of oligomeric $\alpha \mathrm{S}$ determine its membrane interaction?

- What is the mechanism by which oligomeric $\alpha \mathrm{S}$ disrupts lipid membranes?

By answering these questions we hope to gain fundamental understanding of the highly dynamic $\alpha \mathrm{S}$ protein and to offer an insight to the potential role of oligomer membrane interactions in $\mathrm{PD}$. To approach these questions, we have used a wide range of in vitro biophysical and biochemical techniques using synthetic lipid model systems and recombinant $\alpha \mathrm{S}$ grown in E. coli. We realize that both the focus on the amyloid pore hypothesis as well as limiting the experiments to an in vitro environment are a reductionist approach and one should be very cautious in extending the obtained results to in vivo implications for PD. Nonetheless we believe the transient nature and the large heterogeneity inherent to early intermediates in $\alpha \mathrm{S}$ aggregation makes the problem an extremely challenging one to study in a real biological system. Therefore, we are convinced that our biophysical approach to the problem can contribute to assessing the validity and true meaning of the amyloid pore hypothesis in PD.

\section{Acknowledgements}

This work was financed by the Faculty of Science and Technology of the University of Twente, and was a part of the Nanotechnology network in The Netherlands (Nanoned), project number 7921 within the Nanoned programme Bionanosystems. 


\section{References}

(1) de Rijk, M. C., Breteler, M. M., Graveland, G. A., Ott, A., Grobbee, D. E., van der Méche, F. G., and Hofman, A. (1995) Prevalence of Parkinson's disease in the elderly: the Rotterdam Study. Neurology 45, 2143-2146.

(2) Olanow, C. W., Watts, R. L., and Koller, W. C. (2001) An algorithm (decision tree) for the management of Parkinson's disease (2001): treatment guidelines. Neurology 56, S1-S88.

(3) Lewy, F. H. (1912) Paralysis Agitans, in Handbuch der Neurologie, pp 920-933.

(4) Spillantini, M. G., Schmidt, M. L., Lee, V. M., Trojanowski, J. Q., Jakes, R., and Goedert, M. (1997) Alpha-synuclein in Lewy bodies. Nature 388, 839-840.

(5) Chartier-Harlin, M. C., Kachergus, J., Roumier, C., Mouroux, V., Douay, X., Lincoln, S., Levecque, C., Larvor, L., Andrieux, J., Hulihan, M., Waucquier, N., Defebvre, L., Amouyel, P., Farrer, M., and Destée, A. (2004) Alpha-synuclein locus duplication as a cause of familial Parkinson's disease. Lancet 364, 1167-1169.

(6) Krüger, R., Kuhn, W., Müller, T., Woitalla, D., Graeber, M., Kösel, S., Przuntek, H., Epplen, J. T., Schöls, L., and Riess, O. (1998) Ala30Pro mutation in the gene encoding alpha-synuclein in Parkinson's disease. Nat. Genet. 18, 106-108.

(7) Polymeropoulos, M. H., Lavedan, C., Leroy, E., Ide, S. E., Dehejia, A., Dutra, A., Pike, B., Root, H., Rubenstein, J., Boyer, R., Stenroos, E. S., Chandrasekharappa, S., Athanassiadou, A., Papapetropoulos, T., Johnson, W. G., Lazzarini, A. M., Duvoisin, R. C., Di Iorio, G., Golbe, L. I., and Nussbaum, R. L. (1997) Mutation in the alpha-synuclein gene identified in families with Parkinson's disease. Science 276, 2045-2047.

(8) Singleton, A. B., Farrer, M., Johnson, J., Singleton, A., Hague, S., Kachergus, J., Hulihan, M., Peuralinna, T., Dutra, A., Nussbaum, R., Lincoln, S., Crawley, A., Hanson, M., Maraganore, D., Adler, C., Cookson, M. R., Muenter, M., Baptista, M., Miller, D., Blancato, J., Hardy, J., and Gwinn-Hardy, K. (2003) alpha-Synuclein locus triplication causes Parkinson's disease. Science 302, 841. 
(9) Zarranz, J. J., Alegre, J., Gómez-Esteban, J. C., Lezcano, E., Ros, R., Ampuero, I., Vidal, L., Hoenicka, J., Rodriguez, O., Atarés, B., Llorens, V., Gomez Tortosa, E., del Ser, T., Muñoz, D. G., and de Yebenes, J. G. (2004) The new mutation, E46K, of alpha-synuclein causes Parkinson and Lewy body dementia. Ann. Neurol. 55, 164-173.

(10) Cookson, M. R., and van der Brug, M. (2008) Cell systems and the toxic mechanism(s) of alpha-synuclein. Exp. Neurol. 209, $5-11$.

(11) Goldberg, M. S., and Lansbury, P. T., Jr. (2000) Is there a causeand-effect relationship between alpha-synuclein fibrillization and Parkinson's disease? Nat. Cell Biol. 2, E115-E119.

(12) Danzer, K. M., Haasen, D., Karow, A. R., Moussaud, S., Habeck, M., Giese, A., Kretzschmar, H., Hengerer, B., and Kostka, M. (2007) Different species of alpha-synuclein oligomers induce calcium influx and seeding. J. Neurosci. 27, 9220-9232.

(13) Kayed, R., Head, E., Thompson, J. L., McIntire, T. M., Milton, S. C., Cotman, C. W., and Glabe, C. G. (2003) Common structure of soluble amyloid oligomers implies common mechanism of pathogenesis. Science 300, 486-489.

(14) Kayed, R., Sokolov, Y., Edmonds, B., McIntire, T. M., Milton, S. C., Hall, J. E., and Glabe, C. G. (2004) Permeabilization of lipid bilayers is a common conformation-dependent activity of soluble amyloid oligomers in protein misfolding diseases. J. Biol. Chem. 279, 46363-46366.

(15) Lashuel, H. A., Hartley, D., Petre, B. M., Walz, T., and Lansbury, P. T., Jr. (2002) Neurodegenerative disease: amyloid pores from pathogenic mutations. Nature 418, 291.

(16) Volles, M. J., Lee, S. J., Rochet, J. C., Shtilerman, M. D., Ding, T. T., Kessler, J. C., and Lansbury, P. T., Jr. (2001) Vesicle permeabilization by protofibrillar alpha-synuclein: implications for the pathogenesis and treatment of Parkinson's disease. Biochemistry 40, 7812-7819.

(17) Laurents, D. V., and Baldwin, R. L. (1998) Protein folding: matching theory and experiment. Biophys. J. 75, 428-434.

(18) Sosnick, T. R., Mayne, L., Hiller, R., and Englander, S. W. (1994) The barriers in protein folding. Nat. Struct. Biol. 1, 149-156.

(19) Dill, K. A. (1999) Polymer principles and protein folding. Protein Sci. 8, 1166-1180.

(20) Dobson, C. M. (2003) Protein folding and misfolding. Nature 426, 884-890. 
(21) Dill, K. A. (1990) Dominant forces in protein folding. Biochemistry 29, 7133-7155.

(22) Sandberg, W. S., Schlunk, P. M., Zabin, H. B., and Terwilliger, T. C. (1995) Relationship between in vivo activity and in vitro measures of function and stability of a protein. Biochemistry 34 , 11970-11978.

(23) Shoichet, B. K., Baase, W. A., Kuroki, R., and Matthews, B. W. (1995) A relationship between protein stability and protein function. Proc. Natl. Acad. Sci. U. S. A. 92, 452-456.

(24) Goldberg, A. L. (2003) Protein degradation and protection against misfolded or damaged proteins. Nature 426, 895-899.

(25) Hartl, F. U. (1996) Molecular chaperones in cellular protein folding. Nature 381, 571-579.

(26) Bukau, B., Weissman, J., and Horwich, A. (2006) Molecular chaperones and protein quality control. Cell 125, 443-451.

(27) Ciechanover, A. (1994) The ubiquitin-proteasome proteolytic pathway. Cell 79, 13-21.

(28) Balch, W. E., Morimoto, R. I., Dillin, A., and Kelly, J. W. (2008) Adapting proteostasis for disease intervention. Science 319, 916919.

(29) Rattan, S. I. (1996) Synthesis, modifications, and turnover of proteins during aging. Exp. Gerontol. 31, 33-47.

(30) Selkoe, D. J. (2004) Cell biology of protein misfolding: the examples of Alzheimer's and Parkinson's diseases. Nat. Cell Biol. 6, 1054-1061.

(31) Lachmann, H. J., and Hawkins, P. N. (2006) Systemic amyloidosis. Curr. Opin. Pharmacol. 6, 214-220.

(32) Kahn, S. E., Andrikopoulos, S., and Verchere, C. B. (1999) Islet amyloid: a long-recognized but underappreciated pathological feature of type 2 diabetes. Diabetes 48, 241-253.

(33) Prusiner, S. B. (1998) Prions. Proc. Natl. Acad. Sci. U. S. A. 95, 13363-13383.

(34) Serio, T. R., Cashikar, A. G., Kowal, A. S., Sawicki, G. J., Moslehi, J. J., Serpell, L., Arnsdorf, M. F., and Lindquist, S. L. (2000) Nucleated conformational conversion and the replication of conformational information by a prion determinant. Science 289, 1317-1321.

(35) Chiti, F., and Dobson, C. M. (2006) Protein misfolding, functional amyloid, and human disease. Annu. Rev. Biochem. 75, 333-366.

(36) Sipe, J. D., and Cohen, A. S. (2000) Review: history of the amyloid fibril. J. Struct. Biol. 130, 88-98. 
(37) Nelson, R., Sawaya, M. R., Balbirnie, M., Madsen, A. Ø., Riekel, C., Grothe, R., and Eisenberg, D. (2005) Structure of the crossbeta spine of amyloid-like fibrils. Nature 435, 773-778.

(38) Sunde, M., Serpell, L. C., Bartlam, M., Fraser, P. E., Pepys, M. B., and Blake, C. C. (1997) Common core structure of amyloid fibrils by synchrotron X-ray diffraction. J. Mol. Biol. 273, 729739 .

(39) Hirota-Nakaoka, N., Hasegawa, K., Naiki, H., and Goto, Y. (2003) Dissolution of beta2-microglobulin amyloid fibrils by dimethylsulfoxide. J. Biochem. 134, 159-164.

(40) Miake, H., Mizusawa, H., Iwatsubo, T., and Hasegawa, M. (2002) Biochemical characterization of the core structure of alphasynuclein filaments. J. Biol. Chem. 277, 19213-19219.

(41) Braak, H., Del Tredici, K., Rüb, U., de Vos, R. A. I., Steur, E., and Braak, E. (2003) Staging of brain pathology related to sporadic Parkinson's disease. Neurobiol. Aging 24, 197-211.

(42) Samii, A., Nutt, J. G., and Ransom, B. R. (2004) Parkinson's disease. Lancet 363, 1783-1793.

(43) Belin, A. C., and Westerlund, M. (2008) Parkinson's disease: A genetic perspective. FEBS J. 275, 1377-1383.

(44) Dekker, M. C. J., Bonifati, V., and van Duijn, C. M. (2003) Parkinson's disease: piecing together a genetic jigsaw. Brain 126, 1722-1733.

(45) Gorell, J. M., Johnson, C. C., Rybicki, B. A., Peterson, E. L., Kortsha, G. X., Brown, G. G., and Richardson, R. J. (1999) Occupational exposure to manganese, copper, lead, iron, mercury and zinc and the risk of Parkinson's disease. Neurotoxicology 20, 239-247.

(46) Gorell, J. M., Johnson, C. C., Rybicki, B. A., Peterson, E. L., and Richardson, R. J. (1998) The risk of Parkinson's disease with exposure to pesticides, farming, well water, and rural living. Neurology 50, 1346-1350.

(47) Meredith, G. E., and Kang, U. J. (2006) Behavioral models of Parkinson's disease in rodents: A new look at an old problem. Movement Disord. 21, 1595-1606.

(48) Feany, M. B., and Bender, W. W. (2000) A Drosophila model of Parkinson's disease. Nature 404, 394-398.

(49) van Ham, T. J., Thijssen, K. L., Breitling, R., Hofstra, R. M. W., Plasterk, R. H. A., and Nollen, E. A. A. (2008) C-elegans model identifies genetic modifiers of alpha-synuclein inclusion formation during aging. PLoS Gen. 4. 
(50) Kahle, P. J. (2008) alpha-synucleinopathy models and human neuropathology: similarities and differences. Acta Neuropathol. 115, 87-95.

(51) Burke, R. E., Dauer, W. T., and Vonsattel, J. P. G. (2008) A Critical Evaluation of the Braak Staging Scheme for Parkinson's Disease. Ann. Neurol. 64, 485-491.

(52) Parkkinen, L., Kauppinen, T., Pirttilä, T., Autere, J. M., and Alafuzoff, I. (2005) alpha-Synuclein pathology does not predict extrapyramidal symptoms or dementia. Ann. Neurol. 57, 82-91.

(53) Tanaka, M., Kim, Y. M., Lee, G., Junn, E., Iwatsubo, T., and Mouradian, M. M. (2004) Aggresomes formed by alpha-synuclein and synphilin-1 are cytoprotective. J. Biol. Chem. 279, 46254631.

(54) Tompkins, M. M., and Hill, W. D. (1997) Contribution of somal Lewy bodies to neuronal death. Brain Res. 775, 24-29.

(55) Clayton, D. F., and George, J. M. (1998) The synucleins: a family of proteins involved in synaptic function, plasticity, neurodegeneration and disease. Trends Neurosci. 21, 249-254.

(56) Bayer, T. A., Jäkälä, P., Hartmann, T., Egensperger, R., Buslei, R., Falkai, P., and Beyreuther, K. (1999) Neural expression profile of alpha-synuclein in developing human cortex. Neuroreport 10, 2799-2803.

(57) Iwai, A., Masliah, E., Yoshimoto, M., Ge, N. F., Flanagan, L., Desilva, H. A. R., Kittel, A., and Saitoh, T. (1995) The Precursor Protein Of Non-A-Beta Component Of Alzheimers-Disease Amyloid Is A Presynaptic Protein Of The Central-Nervous-System. Neuron 14, 467-475.

(58) Murphy, D. D., Rueter, S. M., Trojanowski, J. Q., and Lee, V. M. Y. (2000) Synucleins are developmentally expressed, and alphasynuclein regulates the size of the presynaptic vesicular pool in primary hippocampal neurons. J. Neurosci. 20, 3214-3220.

(59) Kahle, P. J., Neumann, M., Ozmen, L., Muller, V., Jacobsen, H., Schindzielorz, A., Okochi, M., Leimer, U., van der Putten, H., Probst, A., Kremmer, E., Kretzschmar, H. A., and Haass, C. (2000) Subcellular localization of wild-type and Parkinson's disease-associated mutant alpha-synuclein in human and transgenic mouse brain. J. Neurosci. 20, 6365-6373. 
(60) Yu, S., Li, X., Liu, G., Han, J., Zhang, C., Li, Y., Xu, S., Liu, C., Gao, Y., Yang, H., Uéda, K., and Chan, P. (2007) Extensive nuclear localization of alpha-synuclein in normal rat brain neurons revealed by a novel monoclonal antibody. Neuroscience 145, 539555.

(61) Goers, J., Manning-Bog, A. B., McCormack, A. L., Millett, I. S., Doniach, S., Di Monte, D. A., Uversky, V. N., and Fink, A. L. (2003) Nuclear localization of alpha-synuclein and its interaction with histones. Biochemistry 42, 8465-8471.

(62) Weinreb, P. H., Zhen, W., Poon, A. W., Conway, K. A., and Lansbury, P. T., Jr. (1996) NACP, a protein implicated in Alzheimer's disease and learning, is natively unfolded. Biochemistry 35, 1370913715 .

(63) Davidson, W. S., Jonas, A., Clayton, D. F., and George, J. M. (1998) Stabilization of alpha-synuclein secondary structure upon binding to synthetic membranes. J. Biol. Chem. 273, 9443-9449.

(64) Eliezer, D., Kutluay, E., Bussell, R., Jr., and Browne, G. (2001) Conformational properties of alpha-synuclein in its free and lipidassociated states. J. Mol. Biol. 307, 1061-1073.

(65) Giasson, B. I., Murray, I. V. J., Trojanowski, J. Q., and Lee, V. M. Y. (2001) A hydrophobic stretch of 12 amino acid residues in the middle of alpha-synuclein is essential for filament assembly. J. Biol. Chem. 276, 2380-2386.

(66) Okochi, M., Walter, J., Koyama, A., Nakajo, S., Baba, M., Iwatsubo, T., Meijer, L., Kahle, P. J., and Haass, C. (2000) Constitutive phosphorylation of the Parkinson's disease associated alphasynuclein. J. Biol. Chem. 275, 390-397.

(67) Giasson, B. I., Duda, J. E., Murray, I. V. J., Chen, Q. P., Souza, J. M., Hurtig, H. I., Ischiropoulos, H., Trojanowski, J. Q., and Lee, V. M. Y. (2000) Oxidative damage linked to neurodegeneration by selective alpha-synuclein nitration in synucleinopathy lesions. Science 290, 985-989.

(68) Anderson, J. P., Walker, D. E., Goldstein, J. M., de Laat, R., Banducci, K., Caccavello, R. J., Barbour, R., Huang, J. P., Kling, K., Lee, M., Diep, L., Keim, P. S., Shen, X. F., Chataway, T., Schlossmacher, M. G., Seubert, P., Schenk, D., Sinha, S., Gai, W. P., and Chilcote, T. J. (2006) Phosphorylation of Ser-129 is the dominant pathological modification of alpha-synuclein in familial and sporadic Lewy body disease. J. Biol. Chem. 281, 2973929752 . 
(69) Baba, M., Nakajo, S., Tu, P. H., Tomita, T., Nakaya, K., Lee, V. M. Y., Trojanowski, J. Q., and Iwatsubo, T. (1998) Aggregation of alpha-synuclein in Lewy bodies of sporadic Parkinson's disease and dementia with lewy bodies. Am. J. Pathol. 152, 879-884.

(70) Crowther, R. A., Jakes, R., Spillantini, M. G., and Goedert, M. (1998) Synthetic filaments assembled from C-terminally truncated alpha-synuclein. FEBS Lett. 436, 309-312.

(71) Mouradian, M., Junn, E., Tanaka, M., and Kim, Y. M. (2004) Phosphorylation of alpha-synuclein induces its aggregation. Movement Disord. 19, P42.

(72) Dunker, A. K., Silman, I., Uversky, V. N., and Sussman, J. L. (2008) Function and structure of inherently disordered proteins. Curr. Opin. Struc. Biol. 18, 756-764.

(73) McFarland, M. A., Ellis, C. E., Markey, S. P., and Nussbaum, R. L. (2008) Proteomics Analysis Identifies Phosphorylationdependent alpha-Synuclein Protein Interactions. Mol. Cell. Proteomics 7, 2123-2137.

(74) Tofaris, G. K., and Spillantini, M. G. (2007) Physiological and pathological properties of alpha-synuclein. Cell. Mol. Life Sci. 64, 2194-2201.

(75) Uversky, V. N. (2003) A protein-chameleon: Conformational plasticity of alpha-synuclein, a disordered protein involved in neurodegenerative disorders. J. Biomol. Struct. Dyn. 21, 211-234.

(76) Dyson, H. J., and Wright, P. E. (2005) Intrinsically unstructured proteins and their functions. Nat. Rev. Mol. Cell Biol. 6, 197208.

(77) Chandra, S., Fornai, F., Kwon, H. B., Yazdani, U., Atasoy, D., Liu, X. R., Hammer, R. E., Battaglia, G., German, D. C., Castillo, P. E., and Südhof, T. C. (2004) Double-knockout mice for alphaand beta-synucleins: Effect on synaptic functions. Proc. Natl. Acad. Sci. U. S. A. 101, 14966-14971.

(78) Abeliovich, A., Schmitz, Y., Fariñas, I., Choi-Lundberg, D., Ho, W. H., Castillo, P. E., Shinsky, N., Verdugo, J. M. G., Armanini, M., Ryan, A., Hynes, M., Phillips, H., Sulzer, D., and Rosenthal, A. (2000) Mice lacking alpha-synuclein display functional deficits in the nigrostriatal dopamine system. Neuron 25, 239-252. 
(79) Cabin, D. E., Shimazu, K., Murphy, D., Cole, N. B., Gottschalk, W., McIlwain, K. L., Orrison, B., Chen, A., Ellis, C. E., Paylor, R., Lu, B., and Nussbaum, R. L. (2002) Synaptic vesicle depletion correlates with attenuated synaptic responses to prolonged repetitive stimulation in mice lacking alpha-synuclein. J. Neurosci. 22, 8797-8807.

(80) Dauer, W., Kholodilov, N., Vila, M., Trillat, A. C., Goodchild, R., Larsen, K. E., Staal, R., Tieu, K., Schmitz, Y., Yuan, C. A., Rocha, M., Jackson-Lewis, V., Hersch, S., Sulzer, D., Przedborski, S., Burke, R., and Hen, R. (2002) Resistance of alphasynuclein null mice to the parkinsonian neurotoxin MPTP. Proc. Natl. Acad. Sci. U. S. A. 99, 14524-14529.

(81) Wersinger, C., and Sidhu, A. (2003) Attenuation of dopamine transporter activity by alpha-synuclein. Neurosci. Lett. 340, 189-192.

(82) Ahn, B. H., Rhim, H., Kim, S. Y., Sung, Y. M., Lee, M. Y., Choi, J. Y., Wolozin, B., Chang, J. S., Lee, Y. H., Kwon, T. K., Chung, K. C., Yoon, S. H., Hahn, S. J., Kim, M. S., Jo, Y. H., and Min, D. S. (2002) A-synuclein interacts with phospholipase D isozymes and inhibits pervanadate-induced phospholipase D activation in human embryonic kidney-293 cells. J. Biol. Chem. 277, 1233412342 .

(83) Perrin, R. J., Woods, W. S., Clayton, D. F., and George, J. M. (2000) Interaction of human alpha-Synuclein and Parkinson's disease variants with phospholipids. Structural analysis using sitedirected mutagenesis. J. Biol. Chem. 275, 34393-34398.

(84) Jao, C. C., Der-Sarkissian, A., Chen, J., and Langen, R. (2004) Structure of membrane-bound alpha-synuclein studied by sitedirected spin labeling. Proc. Natl. Acad. Sci. U. S. A. 101, 8331-8336.

(85) Hashimoto, M., Hsu, L. J., Rockenstein, E., Takenouchi, T., Mallory, M., and Masliah, E. (2002) alpha-synuclein protects against oxidative stress via inactivation of the c-Jun N-terminal kinase stress-signaling pathway in neuronal cells. J. Biol. Chem. 277, 11465-11472.

(86) Quilty, M. C., King, A. E., Gai, W. P., Pountney, D. L., West, A. K., Vickers, J. C., and Dickson, T. C. (2006) Alpha-synuclein is upregulated in neurones in response to chronic oxidative stress and is associated with neuroprotection. Exp. Neurol. 199, 249-256. 
(87) Manning-Bog, A. B., McCormack, A. L., Purisai, M. G., Bolin, L. M., and Di Monte, D. A. (2003) alpha-synuclein overexpression protects against paraquat-induced neurodegeneration. J. Neurosci. 23, 3095-3099.

(88) da Costa, C. A., Paitel, E., Vincent, B., and Checler, F. (2002) alpha-Synuclein lowers p53-dependent apoptotic response of neuronal cells - Abolishment by 6-hydroxydopamine and implication for Parkinson's disease. J. Biol. Chem. 277, 50980-50984.

(89) Leng, Y., and Chuang, D. M. (2006) Endogenous alpha-synuclein is induced by valproic acid through histone deacetylase inhibition and participates in neuroprotection against glutamate-induced excitotoxicity. J. Neurosci. 26, 7502-7512.

(90) Kim, T. D., Paik, S. R., Yang, C. H., and Kim, J. (2000) Structural changes in alpha-synuclein affect its chaperone-like activity in vitro. Protein Sci. 9, 2489-2496.

(91) Ostrerova, N., Petrucelli, L., Farrer, M., Mehta, N., Choi, P., Hardy, J., and Wolozin, B. (1999) alpha-Synuclein shares physical and functional homology with 14-3-3 proteins. J. Neurosci. 19, 5782-5791.

(92) Souza, J. M., Giasson, B. I., Lee, V. M. Y., and Ischiropoulos, H. (2000) Chaperone-like activity of synucleins. FEBS Lett. 474, 116-119.

(93) Kim, T. D., Paik, S. R., and Yang, C. H. (2002) Structural and functional implications of C-terminal regions of alpha-synuclein. Biochemistry 41, 13782-13790.

(94) Park, S. M., Jung, H. Y., Kim, T. D., Park, J. H., Yang, C. H., and Kim, J. (2002) Distinct roles of the N-terminal-binding domain and the C-terminal-solubilizing domain of alpha-synuclein, a molecular chaperone. J. Biol. Chem. 277, 28512-28520.

(95) Halliwell, B. (1992) Reactive Oxygen Species And The CentralNervous-System. J. Neurochem. 59, 1609-1623.

(96) Fahn, S., and Cohen, G. (1992) The Oxidant Stress Hypothesis In Parkinsons-Disease - Evidence Supporting It. Ann. Neurol. 32, 804-812.

(97) Bonifati, V., Rizzu, P., van Baren, M. J., Schaap, O., Breedveld, G. J., Krieger, E., Dekker, M. C. J., Squitieri, F., Ibanez, P., Joosse, M., van Dongen, J. W., Vanacore, N., van Swieten, J. C. Brice, A., Meco, G., van Duijn, C. M., Oostra, B. A., and Heutink, P. (2003) Mutations in the DJ-1 gene associated with autosomal recessive early-onset parkinsonism. Science 299, 256-259. 
(98) Migliore, L., and Coppedè, F. (2009) Environmental-induced oxidative stress in neurodegenerative disorders and aging. Mutat. Res. Gen. Tox. En. 674, 73-84.

(99) Cassarino, D. S., and Bennett, J. P. (1999) An evaluation of the role of mitochondria in neurodegenerative diseases: mitochondrial mutations and oxidative pathology, protective nuclear responses, and cell death in neurodegeneration. Brain Res. Rev. 29, 1-25.

(100) Bernheim, H., Birkmayer, W., Hornykiewicz, O., Jellinger, K., and Seitelberger, F., (1973) Brain Dopamine And Syndromes Of Parkinson And Huntington - Clinical, Morphological And Neurochemical Correlations. J. Neurol. Sci. 20, 415-455.

(101) Stokes, A. H., Hastings, T. G., and Vrana, K. E. (1999) Cytotoxic and genotoxic potential of dopamine. J. Neurosci. Res. 55, 659665 .

(102) Conway, K. A., Rochet, J. C., Bieganski, R. M., and Lansbury, P. T. (2001) Kinetic stabilization of the alpha-synuclein protofibril by a dopamine-alpha-synuclein adduct. Science 294, 1346-1349.

(103) Lotharius, J., Barg, S., Wiekop, P., Lundberg, C., Raymon, H. K., and Brundin, P. (2002) Effect of mutant alpha-synuclein on dopamine homeostasis in a new human mesencephalic cell line. J. Biol. Chem. 277, 38884-38894.

(104) Perez, R. G., Waymire, J. C., Lin, E., Liu, J. J., Guo, F. L., and Zigmond, M. J. (2002) A role for alpha-synuclein in the regulation of dopamine biosynthesis. J. Neurosci. 22, 3090-3099.

(105) Parker, W. D., Boyson, S. J., and Parks, J. K. (1989) Abnormalities Of The Electron-Transport Chain In Idiopathic ParkinsonsDisease. Ann. Neurol. 26, 719-723.

(106) Haas, R. H., Nasirian, F., Nakano, K., Ward, D., Pay, M., Hill, R., and Shults, C. W. (1995) Low Platelet Mitochondrial ComplexI And Complex-Ii/Iii Activity In Early Untreated ParkinsonsDisease. Ann. Neurol. 37, 714-722.

(107) Bindoff, L. A., Birchmachin, M. A., Cartlidge, N. E. F., Parker, W. D., and Turnbull, D. M. (1991) Respiratory-Chain Abnormalities In Skeletal-Muscle From Patients With Parkinsons-Disease. J. Neurol. Sci. 104, 203-208.

(108) Schiesling, C., Kieper, N., Seidel, K., and Krüger, R. (2008) Review: Familial Parkinson's disease - genetics, clinical phenotype and neuropathology in relation to the common sporadic form of the disease. Neuropath. Appl. Neurol. 34, 255-271. 
(109) Martin, L. J., Pan, Y., Price, A. C., Sterling, W., Copeland, N. G., Jenkins, N. A., Price, D. L., and Lee, M. K. (2006) Parkinson's disease alpha-synuclein transgenic mice develop neuronal mitochondrial degeneration and cell death. J. Neurosci. 26, 41-50.

(110) Stichel, C. C., Zhu, X. R., Bader, V., Linnartz, B., Schmidt, S., and Lübbert, H. (2007) Mono- and double-mutant mouse models of Parkinson's disease display severe mitochondrial damage. Hum. Mol. Genet. 16, 2377-2393.

(111) Cole, N. B., Dieuliis, D., Leo, P., Mitchell, D. C., and Nussbaum, R. L. (2008) Mitochondrial translocation of alpha-synuclein is promoted by intracellular acidification. Exp. Cell Res. 314, 20762089.

(112) Li, W. W., Yang, R., Guo, J. C., Ren, H. M., Zha, X. L., Cheng, J. S., and Cai, D. F. (2007) Localization of alpha-synuclein to mitochondria within midbrain of mice. Neuroreport 18, 15431546.

(113) Banati, R. B., Daniel, S. E., and Blunt, S. B. (1998) Glial pathology but absence of apoptotic nigral neurons in long-standing Parkinson's disease. Movement Disord. 13, 221-227.

(114) McGeer, P. L., Itagaki, S., Boyes, B. E., and McGeer, E. G. (1988) Reactive Microglia Are Positive For Hla-Dr In The Substantia Nigra Of Parkinsons And Alzheimers-Disease Brains. Neurology 38, 1285-1291.

(115) Hirsch, E. C., and Hunot, S. (2009) Neuroinflammation in Parkinson's disease: a target for neuroprotection? Lancet Neurol. 8, 382-397.

(116) Chen, H. L., Jacobs, E., Schwarzschild, M. A., McCullough, M. L., Calle, E. E., Thun, M. J., and Ascherio, A. (2005) Nonsteroidal antiinflammatory drug use and the risk for Parkinson's disease. Ann. Neurol. 58, 963-967.

(117) Hernán, M. A., Logroscino, G., and Rodríguez, L. A. G. (2006) Nonsteroidal anti-inflammatory drugs and the incidence of Parkinson disease. Neurology 66, 1097-1099.

(118) Bick, R. J., Poindexter, B. J., Kott, M. M., Liang, Y. A., Dinh, K., Kaur, B., Bick, D. L. M., Doursout, M. F., and Schiess, M. C. (2008) Cytokines disrupt intracellular patterns of Parkinson's disease-associated proteins alpha-synuclein, tau and ubiquitin in cultured glial cells. Brain Res. 1217, 203-212. 
(119) Zhang, W., Wang, T. G., Pei, Z., Miller, D. S., Wu, X. F., Block, M. L., Wilson, B., Zhang, W. Q., Zhou, Y., Hong, J. S., and Zhang, J. (2005) Aggregated alpha-synuclein activates microglia: a process leading to disease progression in Parkinson's disease. FASEB J. 19, 533-542.

(120) Park, J. Y., Paik, S. R., Jou, I., and Park, S. M. (2008) Microglial phagocytosis is enhanced by monomeric alpha-synuclein, not aggregated alpha-synuclein: implications for Parkinson's disease. Glia 56, 1215-1223.

(121) Rubinsztein, D. C. (2006) The roles of intracellular proteindegradation pathways in neurodegeneration. Nature 443, 780-786.

(122) Iwatsubo, T., Yamaguchi, H., Fujimuro, M., Yokosawa, H., Ihara, Y., Trojanowski, J. Q., and Lee, V. M. Y. (1996) Purification and characterization of lewy bodies from the brains of patients with diffuse lewy body disease. Am. J. Pathol. 148, 1517-1529.

(123) Kuzuhara, S., Mori, H., Izumiyama, N., Yoshimura, M., and Ihara, Y. (1988) Lewy Bodies Are Ubiquitinated - A Light And Electron-Microscopic Immunocytochemical Study. Acta Neuropathol. 75, 345-353.

(124) McNaught, K. S. P., and Jenner, P. (2001) Proteasomal function is impaired in substantia nigra in Parkinson's disease. Neurosci. Lett. 297, 191-194.

(125) Giasson, B. I., and Lee, V. M. Y. (2001) Parkin and the molecular pathways of Parkinson's disease. Neuron 31, 885-888.

(126) Healy, D. G., Abou-Sleiman, P. M., and Wood, N. W. (2004) Genetic causes of Parkinson's disease: UCHL-1. Cell Tissue Res. 318, 189-194.

(127) Rideout, H. J., Larsen, K. E., Sulzer, D., and Stefanis, L. (2001) Proteasomal inhibition leads to formation of ubiquitin/alphasynuclein-immunoreactive inclusions in PC12 cells. J. Neurochem. 78, 899-908.

(128) McNaught, K. S. P., Perl, D. P., Brownell, A. L., and Olanow, C. W. (2004) Systemic exposure to proteasome inhibitors causes a progressive model of Parkinson's disease. Ann. Neurol. 56, 149-162.

(129) Cuervo, A. M., Stefanis, L., Fredenburg, R., Lansbury, P. T., and Sulzer, D. (2004) Impaired degradation of mutant alpha-synuclein by chaperone-mediated autophagy. Science 305, 1292-1295.

(130) Webb, J. L., Ravikumar, B., Atkins, J., Skepper, J. N., and Rubinsztein, D. C. (2003) alpha-synuclein is degraded by both autophagy and the proteasome. J. Biol. Chem. 278, 25009-25013. 
(131) Lee, H. J., Khoshaghideh, F., Patel, S., and Lee, S. J. (2004) Clearance of alpha-synuclein oligomeric intermediates via the lysosomal degradation pathway. J. Neurosci. 24, 1888-1896.

(132) Vogiatzi, T., Xilouri, M., Vekrellis, K., and Stefanis, L. (2008) Wild type alpha-synuclein is degraded by chaperone-mediated autophagy and macroautophagy in neuronal cells. J. Biol. Chem. 283, 23542-23556.

(133) Snyder, H., Mensah, K., Theisler, C., Lee, J., Matouschek, A., and Wolozin, B. (2003) Aggregated and monomeric alpha-synuclein bind to the S6 ' proteasomal protein and inhibit proteasomal function. J. Biol. Chem. 278, 11753-11759.

(134) Lindersson, E., Beedholm, R., Højrup, P., Moos, T., Gai, W. P., Hendil, K. B., and Jensen, P. H. (2004) Proteasomal inhibition by alpha-synuclein filaments and oligomers. J. Biol. Chem. 279, 12924-12934.

(135) Stefanis, L., Larsen, K. E., Rideout, H. J., Sulzer, D., and Greene, L. A. (2001) Expression of A53T mutant but not wild-type alpha-synuclein in PC12 cells induces alterations of the ubiquitindependent degradation system, loss of dopamine release, and autophagic cell death. J. Neurosci. 21, 9549-9560.

(136) Winklhofer, K. F., Tatzelt, J., and Haass, C. (2008) The two faces of protein misfolding: gain- and loss-of-function in neurodegenerative diseases. EMBO J. 27, 336-349.

(137) Lashuel, H. A., and Lansbury, P. T. (2006) Are amyloid diseases caused by protein aggregates that mimic bacterial pore-forming toxins? Q. Rev. Biophys. 39, 167-201.

(138) Kirkitadze, M. D., Bitan, G., and Teplow, D. B. (2002) Paradigm shifts in Alzheimer's disease and other neurodegenerative disorders: The emerging role of oligomeric assemblies. J. Neurosci. Res. 69, 567-577.

(139) Terry, R. D., Masliah, E., Salmon, D. P., Butters, N., Deteresa, R., Hill, R., Hansen, L. A., and Katzman, R. (1991) Physical Basis Of Cognitive Alterations In Alzheimers-Disease - Synapse Loss Is The Major Correlate Of Cognitive Impairment. Ann. Neurol. 30, 572-580.

(140) Dodart, J. C., Meziane, H., Mathis, C., Bales, K. R., Paul, S. M., and Ungerer, A. (1999) Behavioral disturbances in transgenic mice overexpressing the V717F beta-amyloid precursor protein. Behav. Neurosci. 113, 982-990. 
(141) Moechars, D., Dewachter, I., Lorent, K., Reversé, D., Baekelandt, V., Naidu, A., Tesseur, I., Spittaels, K., Van Den Haute, C., Checler, F., Godaux, E., Cordell, B., and Van Leuven, F. (1999) Early phenotypic changes in transgenic mice that overexpress different mutants of amyloid precursor protein in brain. J. Biol. Chem. 274, 6483-6492.

(142) Lambert, M. P., Barlow, A. K., Chromy, B. A., Edwards, C., Freed, R., Liosatos, M., Morgan, T. E., Rozovsky, I., Trommer, B., Viola, K. L., Wals, P., Zhang, C., Finch, C. E., Krafft, G. A., and Klein, W. L. (1998) Diffusible, nonfibrillar ligands derived from A beta(1-42) are potent central nervous system neurotoxins. Proc. Natl. Acad. Sci. U. S. A. 95, 6448-6453.

(143) Relini, A., Torrassa, S., Rolandi, R., Gliozzi, A., Rosano, C., Canale, C., Bolognesi, M., Plakoutsi, G., Bucciantini, M., Chiti, F., and Stefani, M. (2004) Monitoring the process of HypF fibrillization and liposome permeabilization by protofibrils. J. Mol. Biol. 338, 943-957.

(144) Quist, A., Doudevski, I., Lin, H., Azimova, R., Ng, D., Frangione, B., Kagan, B., Ghiso, J., and Lal, R. (2005) Amyloid ion channels: a common structural link for protein-misfolding disease. Proc. Natl. Acad. Sci. U. S. A. 102, 10427-10432.

(145) Demuro, A., Mina, E., Kayed, R., Milton, S. C., Parker, I., and Glabe, C. G. (2005) Calcium dysregulation and membrane disruption as a ubiquitous neurotoxic mechanism of soluble amyloid oligomers. J. Biol. Chem. 280, 17294-17300.

(146) Kayed, R., and Glabe, C. G. (2006) Conformation-dependent antiamyloid oligomer antibodies, in Amyloid, Prions, And Other Protein Aggregates, Pt C pp 326-344, Elsevier Academic Press Inc, San Diego.

(147) Kourie, J. I., and Henry, C. L. (2002) Ion channel formation and membrane-linked pathologies of misfolded hydrophobic proteins: The role of dangerous unchaperoned molecules. Clin. Exp. Pharmacol. P. 29, 741-753.

(148) Arispe, N., Rojas, E., and Pollard, H. B. (1993) Alzheimer-Disease Amyloid Beta-Protein Forms Calcium Channels In BilayerMembranes - Blockade By Tromethamine And Aluminum. Proc. Natl. Acad. Sci. U. S. A. 90, 567-571. 
(149) Kirik, D., Rosenblad, C., Burer, C., Lundberg, C., Johansen, T. E., Muzyczka, N., Mandel, R. J., and Björklund, A. (2002) Parkinson-like neurodegeneration induced by targeted overexpression of alpha-synuclein in the nigrostriatal system. J. Neurosci. 22, 2780-2791.

(150) van der Putten, H., Wiederhold, K. H., Probst, A., Barbieri, S., Mistl, C., Danner, S., Kauffmann, S., Hofele, K., Spooren, W., Ruegg, M. A., Lin, S., Caroni, P., Sommer, B., Tolnay, M., and Bilbe, G. (2000) Neuropathology in mice expressing human alphasynuclein. J. Neurosci. 20, 6021-6029.

(151) Maries, E., Dass, B., Collier, T. J., Kordower, J. H., and SteeceCollier, K. (2003) The role of alpha-synuclein in Parkinson's disease: Insights from animal models. Nat. Rev. Neurosci. 4, 727-738.

(152) Conway, K. A., Lee, S. J., Rochet, J. C., Ding, T. T., Williamson, R. E., and Lansbury, P. T., Jr. (2000) Acceleration of oligomerization, not fibrillization, is a shared property of both alpha-synuclein mutations linked to early-onset Parkinson's disease: implications for pathogenesis and therapy. Proc. Natl. Acad. Sci. U. S. A. 97, 571-576.

(153) Kostka, M., Högen, T., Danzer, K. M., Levin, J., Habeck, M., Wirth, A., Wagner, R., Glabe, C. G., Finger, S., Heinzelmann, U., Garidel, P., Duan, W., Ross, C. A., Kretzschmar, H., and Giese, A. (2008) Single particle characterization of iron-induced pore-forming alpha-synuclein oligomers. J. Biol. Chem. 283, 10992-11003.

(154) Ding, T. T., Lee, S. J., Rochet, J. C., and Lansbury, P. T., Jr. (2002) Annular alpha-synuclein protofibrils are produced when spherical protofibrils are incubated in solution or bound to brainderived membranes. Biochemistry 41, 10209-10217.

(155) Lashuel, H. A., Petre, B. M., Wall, J., Simon, M., Nowak, R. J., Walz, T., and Lansbury, P. T., Jr. (2002) Alpha-synuclein, especially the Parkinson's disease-associated mutants, forms porelike annular and tubular protofibrils. J. Mol. Biol. 322, 10891102.

(156) Pountney, D. L., Lowe, R., Quilty, M., Vickers, J. C., Voelcker, N. H., and Gai, W. P. (2004) Annular alpha-synuclein species from purified multiple system atrophy inclusions. J. Neurochem. 90, 502-512. 
(157) Volles, M. J., and Lansbury, P. T., Jr. (2002) Vesicle permeabilization by protofibrillar alpha-synuclein is sensitive to Parkinson's disease-linked mutations and occurs by a pore-like mechanism. Biochemistry 41, 4595-4602.

(158) Tsigelny, I. F., Bar-On, P., Sharikov, Y., Crews, L., Hashimoto, M., Miller, M. A., Keller, S. H., Platoshyn, O., Yuan, J. X., and Masliah, E. (2007) Dynamics of alpha-synuclein aggregation and inhibition of pore-like oligomer development by beta-synuclein. FEBS J. 274, 1862-1877.

(159) Zakharov, S. D., Hulleman, J. D., Dutseva, E. A., Antonenko, Y. N., Rochet, J. C., and Cramer, W. A. (2007) Helical alphasynuclein forms highly conductive ion channels. Biochemistry 46, 14369-14379. 



\title{
Chapter 2 \\ Oligomeric $\alpha$-synuclein and its role in aggregation
}

\begin{abstract}
Although the importance of oligomeric intermediates in the aggregation of $\alpha$ synuclein is increasingly recognized, very little is known about these intermediates. The heterogeneity of oligomeric species present in aggregation mixtures, the lack of stability of the intermediates and the low concentrations in which these intermediates are present all pose significant challenges in determining their structural and functional characteristics. In this chapter we have characterized the structural properties of the oligomeric species that are used in the experiments described in this thesis. Furthermore, we have investigated the role of these oligomers in the aggregation process. Our results indicate that $\alpha$-synuclein oligomers possess a $\beta$-sheet secondary structure but are not merely small fibrils. Distinct dye binding properties imply that oligomers are structurally different from fibrils. In addition, oligomers do not seed aggregation as effectively as fibrils. Based on these results we conclude that the oligomers are likely a heterogeneous population of which the majority is off-pathway from the fibrillization process.
\end{abstract}




\section{Introduction}

Similar to other amyloid forming proteins, the fibrillization of $\alpha \mathrm{S}$ follows a sigmoidal growth curve (1). The kinetics are characterized by a lag phase after which fibrillization rapidly proceeds until it reaches a plateau value. The aggregation is often interpreted as a nucleation dependent polymerization process. In such a process, formation of a nucleus is relatively slow. After the formation of the critical nucleus, fibril growth proceeds rapidly. Aggregation can thus be seeded; if fibrillar $\alpha \mathrm{S}$ is added to monomeric $\alpha \mathrm{S}$, aggregation occurs almost instantly (2). Binding of a monomer to fibril ends most likely templates the folding into the $\beta$-sheet amyloid conformation. Fibril breakage is an additional factor that could explain the high growth rate of fibrils. As fibrils grow longer, the chances of breaking increase. Fibril breakage doubles the number of reactive ends and thus accelerates the elongation process (3).

Since $\alpha \mathrm{S}$ is an IDP, aggregation does not occur through unfolding of the native state. Aggregation has been thought to occur through a partially folded intermediate (4). Conditions that favor aggregation, such as a low $\mathrm{pH}$ and high temperatures have been shown to induce secondary structure in the protein. These conditions possibly stabilize a partially folded state of $\alpha \mathrm{S}$, which might be more prone to aggregation (4). Alternatively, it has been proposed that release of long range interactions within the monomer, which makes the hydrophobic region of $\alpha \mathrm{S}$ more accessible, causes the protein to be more aggregation prone (5).

The structures of the earliest aggregation intermediates are not easily accessible by standard techniques. The early intermediates (dimer, trimers, etc) are likely to be in equilibrium with the monomeric protein and can thus not be purified (6). In contrast, the aggregation nucleus is unstable because it has a high propensity to grow through the addition of monomers. Therefore, the size and fold of the nucleus and how exactly it is formed is currently still unknown. Simulation studies have suggested that the initial aggregates are relatively unstructured and collapsed. The micellar-like protein aggregates slowly grow and subsequently reorganize in the amyloid fold (7-9). Both the formation of a micelle as well as conformational reorganization can be rate limiting in such a system. However, such a mechanism still remains to be shown experimentally.

Since oligomers of $\alpha \mathrm{S}$ have been hypothesized to be the potentially toxic species, a number of studies have focused on monitoring oligomer formation during the aggregation process (10-13). However, one has to be cautious in comparing these studies since a rigorous definition for the oligomeric species is lacking. Generally, anything larger than monomeric $\alpha \mathrm{S}$ which is non-fibrillar and soluble is referred to as an oligomer. The first observation of oligomeric $\alpha \mathrm{S}$ comes from atomic force microscopy (AFM) imaging during aggregation, in 
Oligomeric $\alpha$-synuclein and its role in aggregation

which spherical protein particles are observed before the occurrence of fibrils (14). More recently, it has been shown by dynamic light scattering (DLS) studies that there is a transient population of oligomers that disappear upon fibril formation (12).

A number of protocols for the preparation of oligomeric species exist. Volles et al. have used high concentrations of $\alpha \mathrm{S}$ to induce oligomer formation and have purified the resulting oligomers by size exclusion chromatography (15, 16). The resulting oligomers are a heterogeneous population of aggregates with annular and tubular morphologies of around $11 \mathrm{~nm}$ in size (17). These were able to permeabilize lipid vesicles, possibly through a pore-like mechanism (18). Other methods rely on the addition of di- and tri-valent cations to induce oligomerization $(19,20)$. For instance Danzer et al. have used $\mathrm{Fe}^{3+}$ and ethanol to induce the formation of oligomers (6). The resulting aggregates were toxic to cultured cells, but could not be purified since they were in equilibrium with monomeric $\alpha$ S. Finally, larger molecules such as dopamine, lipids, and proteins have been reported to induce oligomer formation (21-24).

In this work we have used a very similar method as Volles et al. (15) to prepare $\alpha \mathrm{S}$ oligomers. First of all, this method does not rely on the addition of multiple components but only requires a high concentration of $\alpha \mathrm{S}$, which reduces the complexity of the system. Furthermore, the protocol results in oligomers that can be purified and are relatively stable. Finally, these oligomers are known to interact with lipid membranes. In this chapter a number of biochemical and biophysical techniques are used to characterize the oligomers formed using this protocol. The results from dye binding studies, seeding experiments and fluorescence correlation spectroscopy (FCS) show that oligomers are structurally different from fibrils. The oligomers did not seed aggregation as effectively as fibrils and were extremely stable. These observations imply that they are off-pathway from the aggregation reaction. However batch to batch differences between oligomers were observed, indicating that the resulting oligomers are heterogeneous in nature.

\section{Results}

One of the challenges in elucidating the structure and function of $\alpha \mathrm{S}$ oligomers is reliable production of the oligomeric species. Little is known about the actual structure of the oligomeric intermediate and its role in the aggregation process. Purification of the oligomers generally does not result in a single homogenous population of an oligomeric state but rather gives a broad distribution of protein aggregates of different sizes (16). We therefore carefully characterized the oligomeric species we produced. 


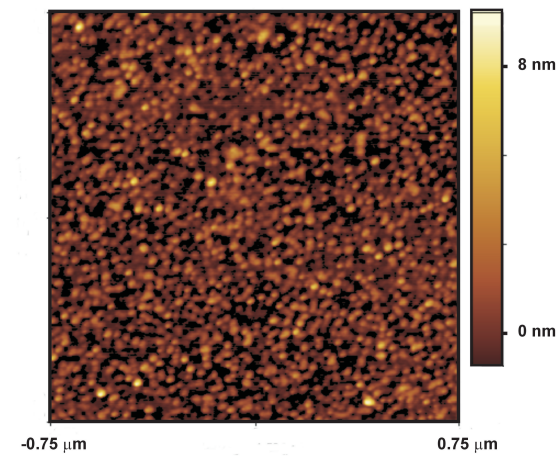

A

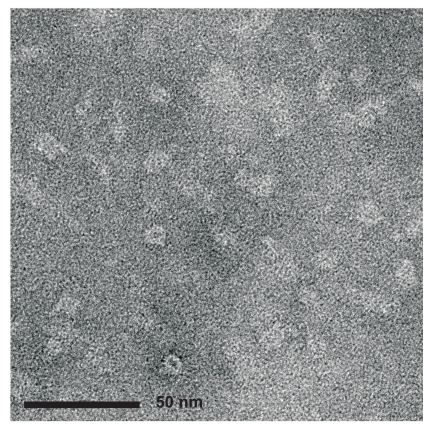

B

Figure 1. Oligomer images (A) Tapping mode AFM in air of $\alpha \mathrm{S}$ oligomers adsorbed to a mica surface (1.5 $\mu \mathrm{m}$ scan size). (B) Transmission electron microscopy image of ammonium molybdate stained oligomers.

In atomic force microscopy (AFM) images the purified oligomers appeared as spherical aggregates with heights in the range of 2-8 nm (Figure 1A). Electron microscopy images of negatively stained oligomers showed spherical and donut shaped particles of around 6-11 nm (Figure 1B). Preparation of oligomeric $\alpha \mathrm{S}$ resulted in low yields (2-4\%), as can be seen in the chromatogram in Figure 2A. The secondary structure of the oligomeric species was characterized using circular dichroism (CD) spectroscopy. The oligomeric intermediate showed a CD spectrum indicative of significant $\beta$-sheet structure. Monomeric $\alpha \mathrm{S}$ showed a characteristic spectrum of an intrinsically disordered protein (Figure $2 \mathrm{~B}$ ). The purified oligomers bound the A11 antibody, which is specific for oligomeric intermediates in the aggregation pathway of many amyloidogenic proteins (Figure 2C) (25). No monomeric $\alpha \mathrm{S}$ could be detected in the purified oligomer solutions using native polyacrylamide gel electrophoresis (PAGE) (Figure 2D). The toxicity of monomeric and oligomeric $\alpha \mathrm{S}$ to SH-SY5Y neuroblastoma cells was assessed by incubating the cells in serum free medium containing different concentrations of $\alpha \mathrm{S}$. Cell viability was measured after 24 hours of incubation using a MST proliferation assay. Both monomeric and oligomeric $\alpha \mathrm{S}$ did not reduce cell viability compared to the control, even at an $\alpha \mathrm{S}$ concentration of $28 \mu \mathrm{M}$ (Figure $2 \mathrm{E}$ ).

In order to gain more structural information, the specificity of oligomeric and fibrillar $\alpha \mathrm{S}$ to a number of dyes that are often used in protein folding studies was tested $(26,27)$. All dyes tested from the anilinonaphthalene-sulfonate 


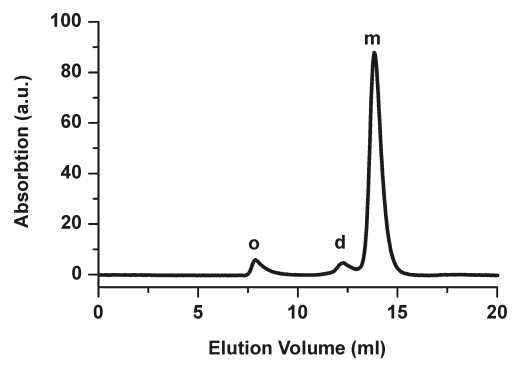

A

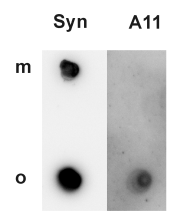

C

D

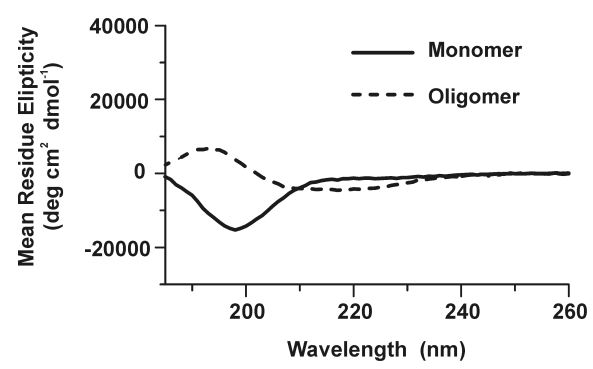

B

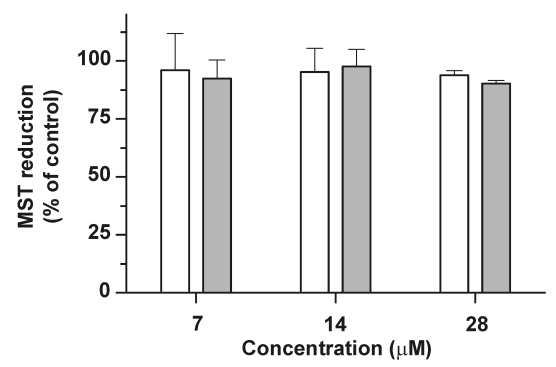

E

Figure 2. Characterization of purified oligomeric $\alpha$ S-wt (A) Size exclusion chromatogram of the purification of $\alpha \mathrm{S}$ oligomers. The oligomeric peak (o) is easily separated from the monomeric $(\mathrm{m})$ peak. Smaller aggregates, possibly dimers (d), sometimes elute just before the monomeric peak. (B) CD spectra of monomeric and oligomeric $\alpha \mathrm{S}$ (C) Immunoblotting with anti- $\alpha \mathrm{S}$ and the A11 anti-oligomeric antibody. The $\alpha \mathrm{S}$ antibody showed both reactivity to monomeric $(\mathrm{m})$ and oligomeric (o) $\alpha \mathrm{S}$. The antibody against oligomeric $\alpha \mathrm{S}$ only showed reactivity to oligomeric $\alpha \mathrm{S}$. (D) Native gradient PAGE with a polyacrylamide gradient form 3 to $17 \%$. Lanes were loaded with fractions from the oligomeric (o), dimeric (d) and monomeric $(\mathrm{m})$ peak of the chromatogram shown in Figure 2A. Ferritin (f) was included as a molecular weight marker $(440 \mathrm{kDa})$. Oligomeric $\alpha \mathrm{S}$ is observed as a broad high molecular weight species above the ferritin band (E) Cell viability compared to the buffer controls of SH-SY5Y neuroblastoma cells after incubation with monomeric (white bars) and oligomeric $\alpha \mathrm{S}$ (grey bars) at different concentrations. The error bars indicate the standard deviation $(n=3)$. 

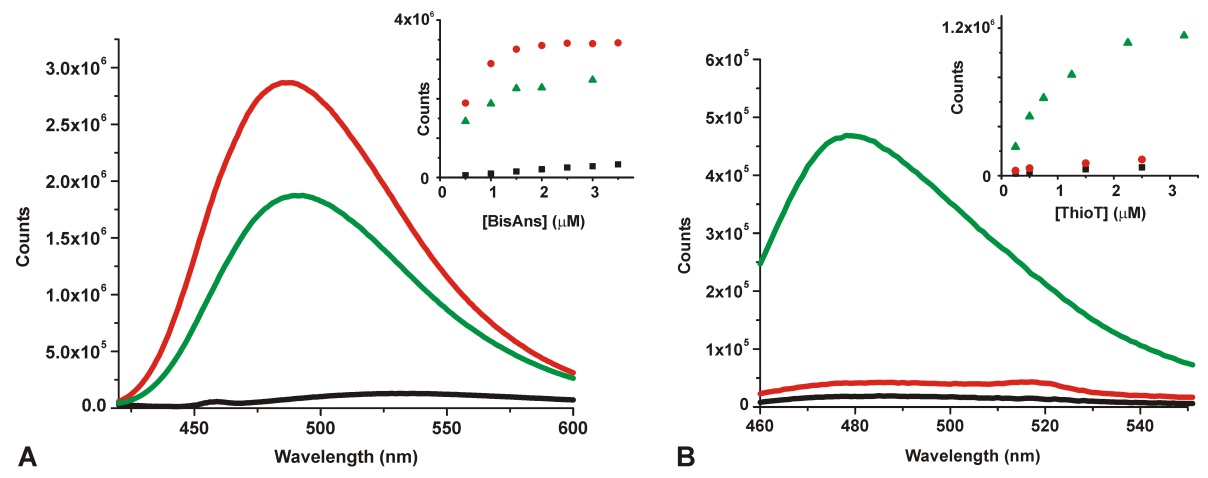

Figure 3. Dye binding properties (A) Bis-ANS (black curve) shows a characteristic blue shift and increase in fluorescence intensity upon addition to both oligomeric (red curve) and fibrillar (green curve) $\alpha \mathrm{S}$. The emission spectrum shown was recorded at $1 \mu \mathrm{M}$ Bis-ANS and at a protein concentration of $0.5 \mu \mathrm{M}$ (equivalent monomer concentration). The inset shows the fluorescence peak intensity when Bis-ANS is titrated to $0.5 \mu \mathrm{M}$ oligomeric (red circles) or fibrillar (green triangles) $\alpha \mathrm{S}$, the black squares indicate the fluorescence of the free dye in solution. (B) Shows similar data for the dye ThioT. Only addition of $\alpha \mathrm{S}$ fibrils induced a marked increase in fluorescence intensity.

family of dyes (ANS, NPN, TNS, Bis-ANS) showed a clear blue shift and increase in fluorescence intensity when added to fibrillar or oligomeric $\alpha \mathrm{S}$ (as is shown for Bis-Ans in Figure 3A). These dyes are known to report on exposed hydrophobic surfaces, characteristic of partially unfolded proteins and molten globules (28). ThioT showed a large increase in fluorescence when added to fibrillar $\alpha \mathrm{S}$ (Figure 3B). Oligomeric $\alpha \mathrm{S}$, only induced a very small increase fluorescence in the ThioT assay. ThioT is thought to be a reasonably specific dye to the characteristic amyloid fold (29). No notable changes in the fluorescence emission was observed in the dyes: DCVJ (amphiphilic, viscosity sensitive) (26), Dapoxyl (amphiphilic, polarity sensitive) (30) and DAUDA (a lipid binding dye) (31). The polarity sensitive dye nile red (32) showed a small change in fluorescence (blue shift and increase in fluorescence intensity) when added to $\alpha \mathrm{S}$ fibrils (data not shown). Monomeric $\alpha \mathrm{S}$ did not induce changes in the fluorescent properties of any of the dyes. The differences in dye binding properties between monomeric, oligomeric and fibrillar $\alpha \mathrm{S}$, indicates that these are structurally distinct species.

Fibrillization is characterized by a lag time after which fibril growth proceeds rapidly. Figure 4 shows an example of an aggregation experiment. The increase in ThioT signal coincides with the loss of soluble $\alpha \mathrm{S}$ and the appear- 
Oligomeric $\alpha$-synuclein and its role in aggregation
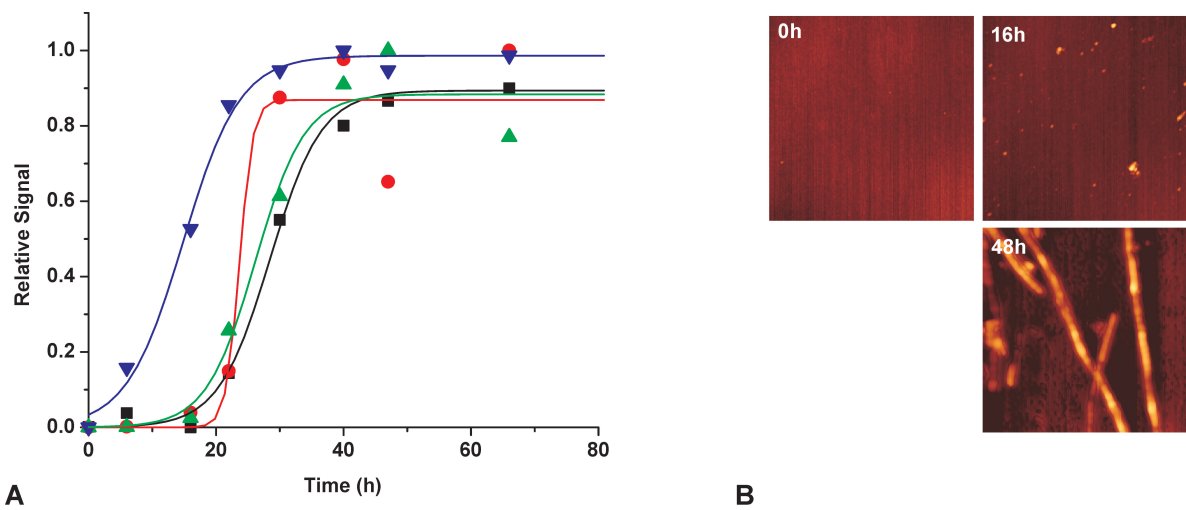

B

Figure 4. $\alpha \mathbf{S}$ aggregation (A) $\alpha \mathrm{S}$ aggregation was followed by ThioT (red circles) and Bis-ANS (green triangles) fluorescence. A distinct lag time phase observed after which the fluorescence intensity increases rapidly. The increase in fluorescence intensity coincides with an increase in the insoluble fraction of $\alpha \mathrm{S}$ (black squares). The peak wavelength of Bis-ANS fluorescence emission (blue inverted triangles) shows a blue shift during the lag phase, indicating that oligomerization does occur. (B) Tapping mode AFM images acquired at various timepoints during the aggregation show that the occurrence of small spherical aggregates precedes the formation of $\alpha \mathrm{S}$ fibrils.

ance of fibrillar morphologies in AFM. Although no ThioT signal is observed during the lag phase, $\alpha \mathrm{S}$ self association and oligomer formation possibly already occur. The results described in the previous paragraph show that Bis-Ans interacts with oligomeric $\alpha$ S. During aggregation the Bis-Ans fluorescence increased at a similar time point as the ThioT fluorescence. This indicates that if oligomers are present during the lag phase their concentration is low. The fluorescence emission peak wavelength is much more sensitive to low concentrations of oligomers. When the peak wavelength is plotted over time, a clear blue shift was observed before the end of the lag phase. Thus during the lag phase formation of oligomers does occur.

In order to elucidate the role of the purified oligomers in the aggregation process, several seeding experiments were performed. In these experiments a small amount of oligomeric or fibrillar $\alpha \mathrm{S}$ is added to a solution of monomeric protein and the aggregation is monitored by ThioT fluorescence. When a small amount of fibrillar seeds of $\alpha \mathrm{S}$ was added to a solution of monomeric $\alpha \mathrm{S}$, aggregation occurred almost instantly (Figure 5A). The seeding effect was less pronounced upon addition of oligomeric $\alpha$ S. Figure 5B shows the characteristic lag time as a function of seed concentration for oligomeric and fibrillar $\alpha \mathrm{S}$. The experiment was repeated a second time with another batch of oligomeric $\alpha \mathrm{S}$ 


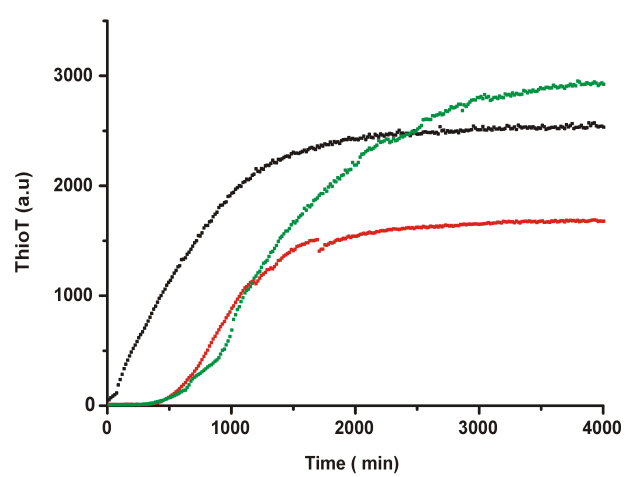

A

Figure 5. Seeded aggregation. Aggregation of $\alpha \mathrm{S}$ was seeded by the addition of fibrillar or oligomeric $\alpha \mathrm{S}$. Aggregation was monitored by ThioT fluorescence. (A) Shows representative curves for $\alpha \mathrm{S}$ aggregation without (green curve) or in the presence of $1 \%$ oligomers (red curve) or fibrils (black curve). (B) The reduction of lag time is plotted as a function of seed concentration, and the error bars indicate the standard deviation $(n=8)$. The lag time was defined as the time it takes for the ThioT fluorescence to reach $10 \%$ of its final value. The addition of fibrils (red circles) greatly reduces the lag time. Addition of oligomers (black squares) did not effectively seed the aggregation of $\alpha \mathrm{S}$. However, another batch of oligomers (green triangles) did show a small seeding effect.

which showed a stronger seeding effect. This effect was more pronounced at high concentrations of oligomers. Compared to fibrillar seeds oligomers are less effective at inducing aggregation.

FCS was used to examine the interaction of $\alpha \mathrm{S}$ monomers with oligomeric $\alpha \mathrm{S}$ and fibrillar seeds more quantitatively. FCS measures the characteristic diffusion time of a fluorescent molecule through a confocal laser spot (see Appendix A for a full explanation on FCS). To prepare fluorescently labeled $\alpha \mathrm{S}$, the dye Alexa 488 (AL488) was attached covalently to a single cysteine mutant of $\alpha \mathrm{S} ; \alpha \mathrm{S}-\mathrm{A} 140 \mathrm{C}$ (alanine to cysteine mutation at residue 140). Fluorescently labeled oligomers can be prepared by adding $\alpha$ S-A140C-AL488 to wild-type (wt) $\alpha \mathrm{S}$ in a 1:7 ratio. Oligomers were further prepared in a manner similar to $\alpha \mathrm{S}$-wt oligomers. Figure $6 \mathrm{~A}$ shows an example of the correlation curves of labeled monomeric and labeled oligomeric $\alpha \mathrm{S}$. Free monomeric $\alpha \mathrm{S}$ is smaller than an $\alpha \mathrm{S}$ oligomer and will therefore diffuse faster. Both curves could be fitted reasonably well using a single component diffusion model. The diffusion time of oligomeric $\alpha \mathrm{S}$ varied slightly from batch to batch, at around 950 to $1000 \mu \mathrm{s}$, compared to $260 \mu \mathrm{s}$ for monomeric $\alpha \mathrm{S}$. From the diffusion time one 
Oligomeric $\alpha$-synuclein and its role in aggregation

can calculate the hydrodynamic radius if the confocal volume is known. The confocal volume was determined by calibration with AL488, where diffusion characteristics have been reported in literature $(33,34)$. Thus the calculated hydrodynamic radii for monomeric and oligomeric $\alpha \mathrm{S}$ were around $2.0 \pm 0.2$ $\mathrm{nm}$ and $7.2 \pm 0.7 \mathrm{~nm}$ respectively.

When fluorescently labeled monomers are added to unlabeled oligomers, monomer-oligomer binding results in a slow diffusing oligomer-bound monomer population and a fast diffusing unbound population. The large separation in diffusion time allows for fitting with a two component diffusion model from which the oligomer bound fraction can be estimated. FCS can thus be used to examine the binding of monomeric $\alpha \mathrm{S}$ to oligomers. To measure if $\alpha \mathrm{S}$ oligomers can still grow and bind $\alpha \mathrm{S}$ monomers, $\alpha \mathrm{S}-140 \mathrm{C}$-AL488 monomer was incubated at a concentration of $9 \mu \mathrm{M}$ in the presence or absence of $1 \mu \mathrm{M}$ $\alpha \mathrm{S}$-wt oligomers. A solution of labeled monomeric $\alpha \mathrm{S}$ with $\alpha \mathrm{S}$ fibrillar seeds $(1 \mu \mathrm{M})$ was examined as a control. At different time points, aliquots were removed and the correlation curves were measured to assess the association of monomeric $\alpha \mathrm{S}$ to the oligomers and fibrillar seeds.

The fraction of monomeric protein obtained by the fitting routine over the course of the experiment is plotted in Figure 6B. The graph indicates that the monomeric protein does not effectively associate with the oligomers. The fraction of monomeric protein in the presence of oligomers does decrease slightly over time but not significantly more than the sample without oligomers present. This indicates that on this time scale, monomer self association can lead to the formation of oligomers. In the presence of fibrillar seeds a clear decrease in signal and thus the amount of monomer in the sample was observed (Figure 6C). Sometimes extremely large bursts in fluorescence intensity occurred, which persisted for a relatively long time (in the order of $0.1-1$ second). The added fibrillar material was most likely too large and heterogeneous to be observed effectively by FCS. Therefore, the resulting FCS curves could not be fitted and monomer fibril binding was not quantified. However, the decrease in signal indicates that the monomers do associate with the fibrils under these conditions.

In principle FCS also allows for assessing the dissociation of oligomeric $\alpha \mathrm{S}$ into monomeric protein or lower order oligomers. To this end fluorescently labeled oligomers were stored over time and the FCS curve was measured at several points in time. Dissociation of a fluorescent monomer will then cause a contribution of a fast diffusing species in the FCS curves. A considerable fraction of monomeric $\alpha \mathrm{S}$ could only be detected upon prolonged storage. The results plotted in Figure 6D show the FCS curve after 4 weeks. By fitting a two component diffusion model it was estimated that around $75 \%$ of the protein was in the oligomeric form. It thus appears that $\alpha \mathrm{S}$ oligomers are not in fast equilibrium with the monomeric protein in solution. 

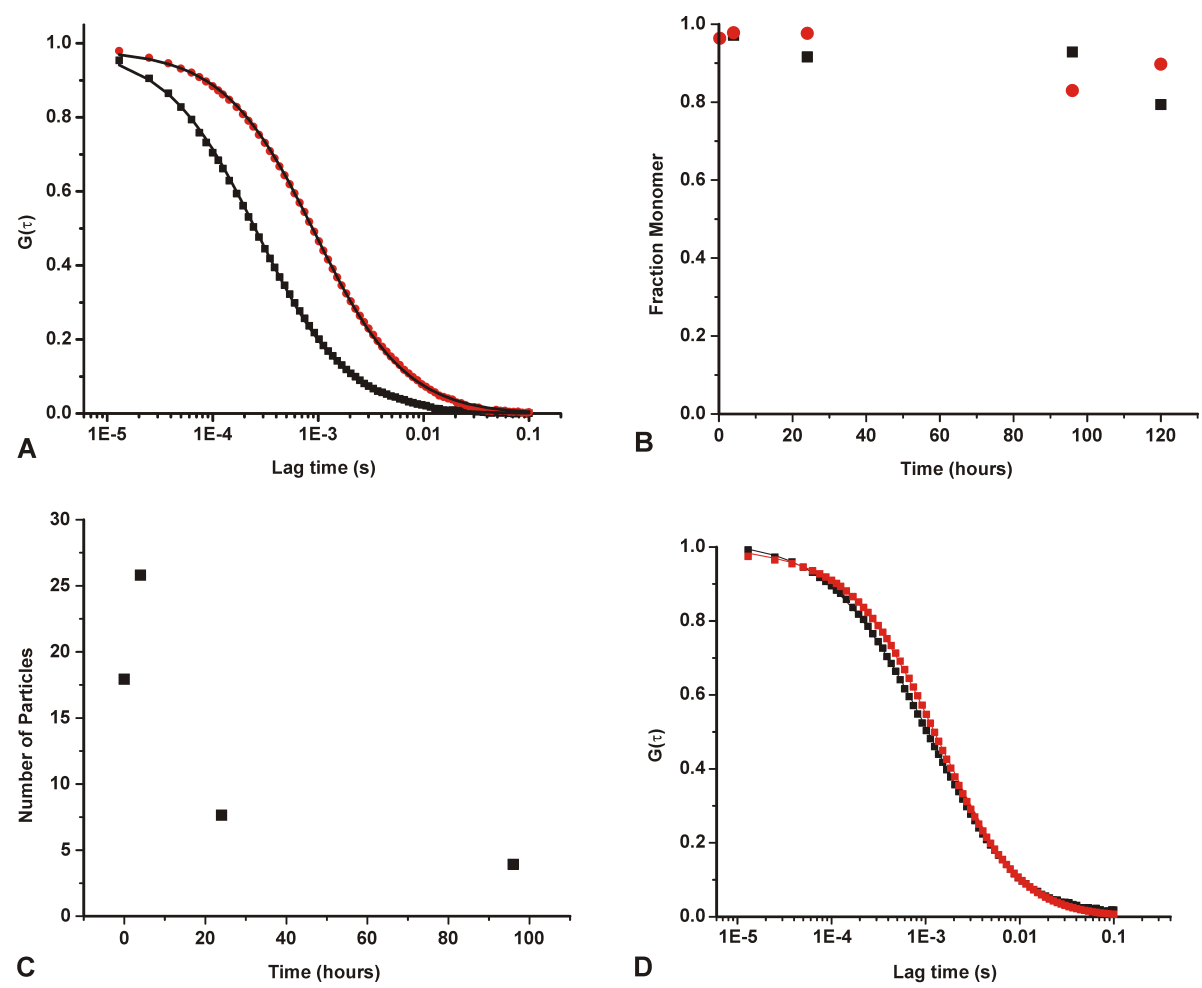

Figure 6. Monomer oligomer binding (A) Correlation curves of $\alpha$ S-A140CAL488 monomers (black squares) and labeled oligomers (red circles). Fitting a single component diffusion model estimates a diffusion time of $\sim 260 \mu$ s for monomeric and $\sim 950-1000 \mu \mathrm{s}$ for oligomeric $\alpha \mathrm{S}$. (B) Monomeric $\alpha \mathrm{S}-\mathrm{A} 140 \mathrm{C}-\mathrm{AL} 488(9 \mu \mathrm{M})$ is incubated either in the presence (red circles) or absence (black squares) of $1 \mu \mathrm{M}$ unlabeled $\alpha \mathrm{S}$ oligomers. A two component diffusion model was fitted to the FCS curves acquired at different time point. The monomeric fraction with a diffusion time of $260 \mu \mathrm{s}$ is plotted in the graph. (C) Monomeric $\alpha$ S-A140C-AL488 $(9 \mu \mathrm{M})$ was incubated in the presence of $1 \mu \mathrm{M}$ unlabeled $\alpha \mathrm{S}$ fibrils. The average number of particles in the focal volume obtained from the fit is plotted as a function of incubation time. (D) Correlation curve of freshly prepared labeled $\alpha \mathrm{S}$ oligomers (red squares) and after 4 weeks at $4{ }^{\circ} \mathrm{C}$ (black squares). After 4 weeks $75 \%$ of the protein is still in the oligomeric form, as estimated by a two component fit. 
Oligomeric $\alpha$-synuclein and its role in aggregation

\section{Discussion}

Oligomers are thought to occur as a transient population during the aggregation of $\alpha \mathrm{S}(11,12)$. Oligomeric $\alpha \mathrm{S}$ is generally prepared by allowing the protein to aggregate for a certain time and isolating the oligomeric intermediates before fibrillization occurs. A multitude of conditions have been applied in different laboratories to induce $\alpha \mathrm{S}$ oligomerization. These different conditions might result in structurally and functionally different oligomers and are therefore difficult to compare.

The structure of oligomeric $\alpha \mathrm{S}$ is experimentally not easily accessible and no detailed structural information is available. Generally, $\alpha \mathrm{S}$ oligomers are reported as spherical protein aggregates with sizes between 2-20 nm $(10,14,17)$. Annular and tubular morphologies are sometimes reported, which are generally around 10-12 $\mathrm{nm}$ in diameter $(17,21,35)$. We have used an increased protein concentration to induce $\alpha \mathrm{S}$ aggregation and have separated the oligomers from monomeric $\alpha \mathrm{S}$ before fibrils are formed. This approach reduces the number of variables in the protocol and is reported to generate oligomers that interact with lipid membranes (15). Oligomeric $\alpha \mathrm{S}$ prepared using this protocol exhibited morphological and structural characteristics similar to those reported by others. Oligomers were rich in $\beta$-sheet structure and the characteristic annular morphology was observed by electron microscopy. Although $\alpha$-helical content has also been reported (10), oligomers are generally considered to possess $\beta$ sheet structure $(12,15)$.

The $\beta$-sheet content could suggest that oligomers already have similar structural elements as fibrils. However, further characterization of $\alpha \mathrm{S}$ oligomers indicated that they are structurally different from fibrils. The dye ThioT did not show an increase in fluorescence intensity when added to $\alpha \mathrm{S}$ oligomers. However, the dye Bis-ANS showed an increase in fluorescence and a blue shift of the peak wavelength. Anilinonaphthalene-sulfonate dyes are known to be more sensitive to the early stages of aggregation possibly through the interaction with $\alpha \mathrm{S}$ oligomers $(11,36)$. Bis-ANS reports on exposed hydrophobic patches and is often used to monitor partially folded or "molten globule" states of proteins (27). The molten globule is a stable collapsed globular state that contains secondary structure elements but lacks the tertiary structure of the native state of the protein. They are commonly observed as folding intermediates of proteins and in the presence of denaturants (37). Remarkably, it has been observed that the molten globule state of several proteins facilitates their

interaction with membranes (38-41). Most likely the exposure of hydrophobic parts and increased conformational flexibility of the protein mediate the lipid interaction of these molten globules. 
Our data in principle allows for an estimate of the size of the oligomers. The oligomers elute in the void fraction of the Superdex 200 column which has a fractionation range up to $600 \mathrm{kDa}$. Under non-denaturing conditions, the oligomers run above the ferritin $(440 \mathrm{kDa})$ band in a polyacrylamide gradient gel. The hydrodynamic radius of oligomeric $\alpha \mathrm{S}$ was estimated to be around 7.2 nm by FCS. Accurate sizing by FCS relies on a good calibration of the confocal volume, which is notoriously difficult to achieve (42). The hydrodynamic radius of monomeric $\alpha \mathrm{S}$ estimated by NMR is around $2.6 \mathrm{~nm}$ (43), much larger than our value of $2.0 \mathrm{~nm}$. Although this value was obtained in a different solution condition (no salt), it is possible that the hydrodynamic radius of the oligomer is also underestimated. If we consider the NMR derived value for monomeric $\alpha \mathrm{S}$ as a calibration standard, the oligomer would have a hydrodynamic radius of around $9.3 \mathrm{~nm}$.

Size exclusion chromatography, FCS and gel electrophoresis all estimate the size of a protein based on its hydrodynamic radius. It is possible to calculate the molecular weight of a protein from its hydrodynamic radius (44). For a correct estimate it is critical to know the structural characteristics of the protein. For instance IDPs are much less densely packed than globular proteins. If we assume that the oligomer has a similar packing density as a molten globule a hydrodynamic radius between 7.2 and $9.3 \mathrm{~nm}$ would correspond to a molecular weight between $524 \mathrm{kDa}$ and 1.1 MDa which corresponds to 37 to 78 monomers of $\alpha \mathrm{S}$. Lashuel et al. (17) have reported a broad size distribution for purified oligomers from roughly $200 \mathrm{kDa}$ to $800 \mathrm{kDa}$. The oligomeric particles with annular morphologies were estimated to have a molecular weight of around 350 $\mathrm{kDa}$. Our estimate is somewhat larger, but together these data suggest that in order to be thermodynamically stable, oligomers need to be of a certain size, much larger than dimers and other low order oligomers.

In addition to the heterogeneity in the size distribution of oligomeric species, possibly there exists a structural and functional heterogeneity. Although the aggregation pathway into amyloid fibrils is the dominant one under physiological salt and $\mathrm{pH}$ conditions, alternative pathways very likely exist. $\alpha \mathrm{S}$ has also been reported to form amorphous aggregates (45) that are different from fibrils, and in addition, off-pathway oligomers have also been observed (6). To assess where the $\alpha \mathrm{S}$ oligomers obtained using our protocol fit into the aggregation scheme, we have performed seeding experiments. The results indicate that the $\alpha \mathrm{S}$ oligomers did not seed aggregation as effectively as fibrillar $\alpha \mathrm{S}$. We did observe batch to batch differences in seeding ability of the oligomers and a seeding effect was observed at high oligomer concentration. This could suggest that the oligomers are structurally heterogeneous. A small fraction of the purified oligomers in an amyloid-like fold is sufficient to explain the observed seeding effects at high oligomer concentration. The majority of the purified 
Oligomeric $\alpha$-synuclein and its role in aggregation

oligomers might then be off-pathway from fibrillization and therefore do not seed the aggregation. The relative size of two populations could be different from batch to batch. An alternative explanation would be that oligomers are able to reorganize and slowly assume the amyloid fold, which is for instance predicted from simulations (8). This conformational change in the oligomer could then be the rate limiting step in the aggregation.

Another way to assess the role of the oligomers in the aggregation is to directly study the interaction between $\alpha \mathrm{S}$ oligomers and $\alpha \mathrm{S}$ monomers. If oligomers were on-pathway to fibril formation, monomeric $\alpha \mathrm{S}$ should be able to associate with an oligomer. Monomer-oligomer association was measured quantitatively using FCS. The data from this experiment showed that monomeroligomer association most likely does not occur or at least is not stronger than monomer-monomer association. In contrast, monomeric $\alpha \mathrm{S}$ showed a strong interaction with the fibrillar seeds and was in this case rapidly lost from the solution. These data support the low seeding ability of $\alpha \mathrm{S}$ oligomers in the seeded aggregation experiment. The fact that we do not see any considerable monomer loss in this experiment on a relatively long time scale also indicates that if oligomer refolding happens it is at least a very slow process. The duration of the lag time during monomer aggregation experiments is faster (generally around 1 to 3 days at $100 \mu \mathrm{M} \alpha \mathrm{S}$ ). We therefore believe it is unlikely that seed formation occurs through a conformational change in these large oligomers. Finally, oligomers appeared extremely stable and disassociation of the oligomers into monomeric $\alpha$ S occurred on the time scale of weeks. Only in the presence of high concentrations of guanidine hydrochloride we have observed a full dissociation into monomeric $\alpha \mathrm{S}(24)$. Therefore we conclude that off-pathway oligomers are most likely not a transient population.

Summarizing, the $\alpha \mathrm{S}$ oligomers prepared using our protocol possess similar properties to those previously reported in the literature. They have a $\beta$-sheet secondary structure, but are structurally different from $\alpha \mathrm{S}$ fibrils. Most likely the majority of oligomers is off-pathway from the fibrillization and oligomers are not in fast equilibrium with monomeric $\alpha \mathrm{S}$.

\section{Materials and Methods}

Expression and purification of $\alpha S$. Expression of the human $\alpha \mathrm{S}$-wt and mutant $\alpha \mathrm{S}$, with a single alanine to cysteine substitution at residue 140 ( $\alpha \mathrm{S}$-A140C) was performed in E. Coli B121 (DE3) using the pT7-7 based expression system. Bacterial cell pellets were harvested after IPTG induction and resuspended in $10 \mathrm{mM}$ Tris-HCl, PH 8.0, $1 \mathrm{mM}$ EDTA and $1 \mathrm{mM}$ Pefabloc protease inhibitor cocktail. Cells were disrupted by sonication followed by centrifugation for 30 minutes at $10,000 \times \mathrm{g}$ and $4^{\circ} \mathrm{C}$ to remove cellular debris. Nucleotides were 
removed using streptomycin sulphate $(1 \% \mathrm{w} / \mathrm{v})$ and subsequent centrifugation for 30 minutes at $13,500 \times \mathrm{g}$ and $4^{\circ} \mathrm{C}$. $\alpha \mathrm{S}$ was precipitated by ammonium sulfate $(0.3 \mathrm{~g} / \mathrm{ml})$ and pelleted by centrifugation for 30 minutes at $13,500 \times$ $\mathrm{g}$ and $4^{\circ} \mathrm{C}$. The pellet was dissolved in $10 \mathrm{mM}$ Tris- $\mathrm{HCl}, \mathrm{pH} 7.4,1 \mathrm{mM}$ Pefabloc and filtered through a $0.2 \mu \mathrm{m}$ membrane. The resulting solution was loaded onto a Resource Q anion exchange column (GE) on an Akta Basic chromatography system (GE). Fractions collected during elution with a salt gradient from 0-1 $\mathrm{M}$ were assayed for the presence of $\alpha \mathrm{S}$ protein by SDS-PAGE followed by Coomassie staining. Fractions containing $\alpha \mathrm{S}$ were pooled, dialyzed against $10 \mathrm{mM}$ HEPES, $50 \mathrm{mM} \mathrm{NaCl}$, pH 7.4 and concentrated to $250 \mu \mathrm{M}$. For the cysteine mutant the purification protocol was performed in the presence of $1 \mathrm{mM}$ DL-Dithiothreitol (DTT). Protein concentrations were determined by measuring the absorbance at $275 \mathrm{~nm}$ using an extinction coefficient of 5,600 $\mathrm{M}^{-1} \mathrm{~cm}^{-1}$. Purified protein was stored at $-80^{\circ} \mathrm{C}$ in $0.5 \mathrm{ml}$ aliquots until further use.

$\alpha S$ labeling. Prior to labeling, $\alpha \mathrm{S}-\mathrm{A} 140 \mathrm{C}$ was reduced with a five-fold molar excess of DTT for 30 minutes at room temperature. The samples were desalted with Pierce Zeba desalting columns, followed by the addition of a two-fold molar excess of AL488 C5 maleimide dye (Invitrogen) and incubated for one hour in the dark at room temperature. Free label was removed using two desalting steps. The protein labeling efficiency was estimated to be $\sim 80 \%$ from the absorption spectrum.

Purification of $\alpha S$ oligomers. To prepare the $\alpha \mathrm{S}$ oligomeric species, 250 $\mu \mathrm{M}$ stock solutions of $\alpha \mathrm{S}$ monomers in $10 \mathrm{mM}$ HEPES $\mathrm{pH} 7.4$, were dried in a vacuum evaporator and dissolved using MilliQ water at a protein concentration of $1 \mathrm{mM}$. The protein solution was incubated in an Eppendorf thermo mixer for 18 hours at room temperature at $300 \mathrm{rpm}$. Subsequently the mixture was incubated at $37^{\circ} \mathrm{C}$ for 2 hours without agitation. After filtration through a 0.2 $\mu \mathrm{m}$ spin-X centrifugal filter (Corning) to remove possible large aggregates, the oligomeric species were separated from the monomeric protein by size-exclusion chromatography on a Superdex 200 (GE) gel filtration column using $10 \mathrm{mM}$ HEPES pH 7.4, $150 \mathrm{mM} \mathrm{NaCl}$ as eluant. Fractions containing the $\alpha \mathrm{S}$ oligomers, judged from UV absorption spectra, were pooled and concentrated using a Vivaspin (Sartorius) concentrator with a $10 \mathrm{kDa}$ molecular weight cutoff. The protein concentration was estimated by measuring the absorption at $275 \mathrm{~nm}$. A correction for scattering was applied as in (46), which was generally around $10-15 \%$ in magnitude.

Preparation of labeled oligomers. $\alpha \mathrm{S}$-wt was mixed with $\alpha \mathrm{S}-\mathrm{A} 140 \mathrm{C}-\mathrm{AL} 488$ at a 7:1 ratio in $10 \mathrm{mM}$ Tris- $\mathrm{HCl}, \mathrm{pH} 7.4$ and lyophilized. Oligomer preparation was performed similar as the preparation of $\alpha \mathrm{S}$-wt oligomers. The oligomer concentration and final labeling ratio were determined from the absorption 
Oligomeric $\alpha$-synuclein and its role in aggregation

spectra. Generally a 10-15\% final labeling efficiency was obtained.

Atomic Force Microscopy. AFM images were obtained using tapping mode AFM in air, on custom-built equipment (47). Samples were prepared by placing a $5 \mu \mathrm{l}$ drop of protein solution on a piece of freshly cleaved mica. The protein was allowed to adsorb to the surface by incubating for 5 minutes in a humid environment. After incubation, excess liquid was removed and the sample was washed twice with $20 \mu \mathrm{l}$ MilliQ water which was immediately blotted off. Finally, the sample was dried under a gentle stream of nitrogen gas.

Electron Microscopy. A $5 \mu \mathrm{l}$ aliquot of protein solution was placed on a carbon coated copper grid and was allowed to adsorb to the grid during a 5 minutes incubation period under a humid atmosphere to prevent complete drying. Drops of MilliQ water and $2 \%$ ammonium molybdate were placed on a piece of parafilm. The grid was washed by touching the drop of MilliQ water which was immediately blotted off. The grid was then stained by touching a drop of staining solution which was blotted off after 30 seconds. The samples were imaged in a Philips CM300ST-FEG transmission electron microscope.

Circular dichroism spectroscopy. CD spectra were recorded using a Jasco 715 spectropolarimeter using a $0.05 \mathrm{~cm}$ path length quartz cuvette. Monomeric and oligomeric species of $\alpha \mathrm{S}$ at $10 \mu \mathrm{M}$ concentration were prepared in $5 \mathrm{mM}$ Tris-Borate $\mathrm{pH} 7.5$ buffer.

Immunoblotting. $2 \mu \mathrm{l}$ of monomeric and oligomeric $\alpha \mathrm{S}(20 \mu \mathrm{M}$ both) were adsorbed to a nitrocellulose membrane. After blocking for 2 hours with $10 \%$ non fat dry milk, $10 \mathrm{mM}$ Tris- $\mathrm{HCl}$ pH 7.4, $150 \mathrm{mM} \mathrm{NaCl}, 0.05 \%$ Tween20, dot blots were incubated for 2 hour with either the A11 anti oligomeric antibody (Chemicon) or an anti $\alpha \mathrm{S}$ antibody (Sigma) in $5 \%$ non fat dry milk, 10 $\mathrm{mM}$ Tris- $\mathrm{HCl} \mathrm{pH} 7.4,150 \mathrm{mM} \mathrm{NaCl}, 0.05 \%$ Tween20. Subsequently blots were incubated for 1 hour with a goat-anti-mouse secondary antibody coupled to horse radish peroxide (Sigma). Binding to the blots was detected on a Nikon imaging station from the luminescence after adding SuperSignal pico substrate (Pierce).

Native gel electrophoresis. Polyacrylamide gels were cast with a linear gradient from 3 to $17 \%$. Gels were run under non-denaturing conditions. Ferritin $(440 \mathrm{kDa})$ was loaded as a molecular weight marker. Protein bands were visualized using silver staining.

Cell Culture. Human SH-SY5Y neuroblastoma cells were maintained in IMDM (GibcoBRL) supplemented with $10 \%$ heat-inactivated FBS (GibcoBRL) at $37^{\circ} \mathrm{C}, 5.0 \% \mathrm{CO}_{2}$ in a humidified incubator. Cells were plated in a 96 well plate (Corning) at 50000 cells per well. After 24 hours the medium was replaced with serum free medium and monomeric and oligomeric $\alpha \mathrm{S}$ were added at several concentrations. After 24 hours a MTS proliferation assay (Promega) was performed. Toxicity was assessed by measuring the amount of 
soluble formazan produced in each well by measuring the light absorption at $490 \mathrm{~nm}$ in a platereader (Tecan). Cell viability was expressed as the percentage of formazan produced compared to the control wells. Measurements were performed in triplicate.

Dye binding experiments. 8-Anilino-1-naphthalenesulfonic acid (ANS), 4,4Dianilino-1,1-binaphthyl-5,5-disulfonic acid (Bis-ANS), 9-(2,2-Dicyanovinyl) julolidine (DCVJ), nile red, Thioflavin-T (ThioT), N-Phenyl-1-naphthylamine (NPN) and 2-(p-Toluidino)-6-naphthalenesulfonic acid (TNS) were all obtained from Sigma, the dyes DAUDA and Dapoxyl were obtained from Invitrogen. Fluorescence emission spectra were recorded on a Fluoromax 4 (Jobin Yvon) fluorimeter. Small aliquots from a $200 \mu \mathrm{M}$ concentrated dyes stock in methanol were added to a solution of $1 \mu \mathrm{M}$ oligomeric or fibrillar $\alpha \mathrm{S}$. After each addition a spectrum was recorded. Bis-ANS was excited at $395 \mathrm{~nm}$ and ThioT was excited at $440 \mathrm{~nm}$, The excitation and emission slits were set to $2 \mathrm{~nm}$ and 5 $\mathrm{nm}$ respectively. Emission spectra were corrected for the background signal recorded in the absence of dye.

Aggregation experiment. Around $80 \mu \mathrm{M}$ of $\alpha \mathrm{S}-\mathrm{wt}$ in $10 \mathrm{mM}$ Tris-HCl, $\mathrm{pH}$ 7.4 and $40 \mathrm{mM} \mathrm{NaCl}$ was incubated at $37^{\circ} \mathrm{C}$. Agitation was provided with a Teflon coated magnetic stir bar at $300 \mathrm{rpm}$. At several time points protein was removed from the aggregation mixture to perform several assays. Aggregation was assessed by the dyes ThioT and Bis-ANS. Fluorescence from these dyes was measured similar as in the dye binding experiment explained above. For these measurements $5 \mu \mathrm{l}$ of the aggregation mixture was added to $500 \mu \mathrm{l}$ of $1 \mu \mathrm{M}$ of dye solution. To asses the amount of soluble material, $50 \mu \mathrm{l}$ of the aggregation mixture was centrifuged for 60 minutes at $21,000 \times$ g. Subsequently the supernatant was removed and diluted 4 times before measuring the absorption spectrum from which the protein concentration in the supernatant was estimated. Furthermore $5 \mu \mathrm{l}$ aliquots were removed at several time points for AFM imaging.

Seeding experiment. In the seeding experiments $65 \mu \mathrm{M} \alpha \mathrm{S}$ in $10 \mathrm{mM}$ HEPES $\mathrm{pH} 7.4$ and $50 \mathrm{mM} \mathrm{NaCl}$ in the presence of $40 \mu \mathrm{M}$ ThioT was incubated in a Tecan Saphire platereader. Incubation was performed in a 96 well plate at $250 \mu \mathrm{l}$ volume per well at $37^{\circ} \mathrm{C}$ and with orbital shaking as agitation. ThioT fluorescence was measured every 15 minutes, with excitation at $446 \mathrm{~nm}$ and the emission was measured at $485 \mathrm{~nm}$ (20 nm bandwidths). To asses seeding, oligomers were added as different weight percentages to a constant amount of total protein. For comparison, different percentages of fibrillar seeds were also added. For each measurement at least 8 wells were used. Fibrillar seeds were prepared by sonication of a $\alpha \mathrm{S}$ fibrils for 1 minute in a bath sonicator.

Fluorescence Correlation spectroscopy. FCS experiments and calculation of hydrodynamic radius and molecular weight were performed as described in 
Oligomeric $\alpha$-synuclein and its role in aggregation

Appendix A.

Monomer-oligomer binding. To assess the interaction between oligomeric $\alpha \mathrm{S}$ and monomeric $\alpha \mathrm{S}, 9 \mu \mathrm{M} \alpha \mathrm{S}-\mathrm{A} 140 \mathrm{C}-\mathrm{Al} 488$ was incubated with or without $1 \mu \mathrm{M} \alpha \mathrm{S}$-wt oligomers or fibrils seeds. Incubation was performed in an Eppendorf thermomixer at $37^{\circ} \mathrm{C}$ and $1000 \mathrm{rpm}$. At different time points an aliquot was removed for FCS analysis. Before recording the FCS curves the protein was diluted to $50 \mathrm{nM}$ and a $100 \mu \mathrm{l}$ drop was deposited on a cover glass. For FCS measurements cover glasses were treated to prevent adsorption of the protein to the surface. After cleaning in piranha solution for 15 minutes (3:1 ratio of concentrated sulfuric acid to $30 \%$ hydrogen peroxide solution) and rinsing with ultrapure water, the slides were pacified with a PEG-silane compound. Cover glasses were incubated overnight in $\sim 0.1 \%$ weight/volume 2-[Methoxy(polyethyleneoxy)propyl] trimethoxysilane (ABCR) in toluene under a nitrogen atmosphere. After this procedure the cover glasses were rinsed with fresh toluene and subjected to a 1 minute sonication. The resulting FCS curves were fitted with the two component diffusion model (Appendix A, equation 4). Before measuring monomer-oligomer binding, the diffusion time of the free protein was determined and kept fixed in the fitting of the correlation curves in the subsequent binding experiment. The diffusion time of the oligomer bound protein was not fixed in the fitting of the FCS curves, only a lower bound of $900 \mu$ s was set.

\section{Acknowledgements}

Purification and mutagenesis of $\alpha \mathrm{S}$ protein used for the experiments described in this chapter and throughout the thesis were performed by Marloes ten HaaffKolkman, Yvonne Kraan and Kirsten van Leijenhorst-Groener.

\section{References}

(1) Lomakin, A., Chung, D. S., Benedek, G. B., Kirschner, D. A., and Teplow, D. B. (1996) On the nucleation and growth of amyloid beta-protein fibrils: Detection of nuclei and quantitation of rate constants. Proc. Natl. Acad. Sci. U. S. A. 93, 1125-1129.

(2) Wood, S. J., Wypych, J., Steavenson, S., Louis, J. C., Citron, M., and Biere, A. L. (1999) alpha-synuclein fibrillogenesis is nucleation-dependent - Implications for the pathogenesis of Parkinson's disease. J. Biol. Chem. 274, 19509-19512. 
(3) Xue, W. F., Homans, S. W., and Radford, S. E. (2008) Systematic analysis of nucleation-dependent polymerization reveals new insights into the mechanism of amyloid self-assembly. Proc. Natl. Acad. Sci. U. S. A. 105, 8926-8931.

(4) Uversky, V. N., Li, J., and Fink, A. L. (2001) Evidence for a partially folded intermediate in alpha-synuclein fibril formation. J. Biol. Chem. 276, 10737-10744.

(5) Bertoncini, C. W., Jung, Y. S., Fernandez, C. O., Hoyer, W., Griesinger, C., Jovin, T. M., and Zweckstetter, M. (2005) Release of long-range tertiary interactions potentiates aggregation of natively unstructured alpha-synuclein. Proc. Natl. Acad. Sci. U. S. A. 102, 1430-1435.

(6) Danzer, K. M., Haasen, D., Karow, A. R., Moussaud, S., Habeck, M., Giese, A., Kretzschmar, H., Hengerer, B., and Kostka, M. (2007) Different species of alpha-synuclein oligomers induce calcium influx and seeding. J. Neurosci. 27, 9220-32.

(7) Auer, S., Meersman, F., Dobson, C. M., and Vendruscolo, M. (2008) A Generic Mechanism of Emergence of Amyloid Protofilaments from Disordered Oligomeric Aggregates. PLoS Comput. Biol. 4.

(8) Cheon, M., Chang, I., Mohanty, S., Luheshi, L. M., Dobson, C. M., Vendruscolo, M., and Favrin, G. (2007) Structural reorganisation and potential toxicity of oligomeric species formed during the assembly of amyloid fibrils. PLoS Comput. Biol. 3, 1727-1738.

(9) Pellarin, R., and Caflisch, A. (2006) Interpreting the aggregation kinetics of amyloid peptides. J. Mol. Biol. 360, 882-892.

(10) Apetri, M. M., Maiti, N. C., Zagorski, M. G., Carey, P. R., and Anderson, V. E. (2006) Secondary structure of alpha-synuclein oligomers: Characterization by Raman and atomic force microscopy. J. Mol. Biol. 355, 63-71.

(11) Dusa, A., Kaylor, J., Edridge, S., Bodner, N., Hong, D. P., and Fink, A. L. (2006) Characterization of oligomers during alpha-synuclein aggregation using intrinsic tryptophan fluorescence. Biochemistry 45, 2752-2760.

(12) Kaylor, J., Bodner, N., Edridge, S., Yamin, G., Hong, D. P., and Fink, A. L. (2005) Characterization of oligomeric intermediates in alpha-synuclein fibrillation: FRET studies of Y125W/Y133F/Y136F alpha-synuclein. J. Mol. Biol. 353, 357372 . 
Oligomeric $\alpha$-synuclein and its role in aggregation

(13) Thirunavukkuarasu, S., Jares-Erijman, E. A., and Jovin, T. M. (2008) Multiparametric fluorescence detection of early stages in the amyloid protein aggregation of pyrene-labeled alphasynuclein. J. Mol. Biol. 378, 1064-1073.

(14) Conway, K. A., Lee, S. J., Rochet, J. C., Ding, T. T., Williamson, R. E., and Lansbury, P. T., Jr. (2000) Acceleration of oligomerization, not fibrillization, is a shared property of both alpha-synuclein mutations linked to early-onset Parkinson's disease: implications for pathogenesis and therapy. Proc. Natl. Acad. Sci. U. S. A. 97, 571-576.

(15) Volles, M. J., Lee, S. J., Rochet, J. C., Shtilerman, M. D., Ding, T. T., Kessler, J. C., and Lansbury, P. T., Jr. (2001) Vesicle permeabilization by protofibrillar alpha-synuclein: implications for the pathogenesis and treatment of Parkinson's disease. Biochemistry 40, 7812-7819.

(16) Lashuel, H. A., and Grillo-Bosch, D. (2005) In vitro preparation of prefibrillar intermediates of amyloid-beta and alpha-synuclein. Method. Mol. Biol. 299, 19-33.

(17) Lashuel, H. A., Petre, B. M., Wall, J., Simon, M., Nowak, R. J., Walz, T., and Lansbury, P. T., Jr. (2002) Alpha-synuclein, especially the Parkinson's disease-associated mutants, forms porelike annular and tubular protofibrils. J. Mol. Biol. 322, 10891102.

(18) Volles, M. J., and Lansbury, P. T., Jr. (2002) Vesicle permeabilization by protofibrillar alpha-synuclein is sensitive to Parkinson's disease-linked mutations and occurs by a pore-like mechanism. Biochemistry 41, 4595-4602.

(19) Lowe, R., Pountney, D. L., Jensen, P. H., Gai, W. P., and Voelcker, N. H. (2004) Calcium(II) selectively induces alpha-synuclein annular oligomers via interaction with the C-terminal domain. Protein Sci. 13, 3245-3252.

(20) Kostka, M., Högen, T., Danzer, K. M., Levin, J., Habeck, M., Wirth, A., Wagner, R., Glabe, C. G., Finger, S., Heinzelmann, U., Garidel, P., Duan, W., Ross, C. A., Kretzschmar, H., and Giese, A. (2008) Single particle characterization of iron-induced pore-forming alpha-synuclein oligomers. J. Biol. Chem. 283, 10992-11003.

(21) Ding, T. T., Lee, S. J., Rochet, J. C., and Lansbury, P. T. (2002) Annular alpha-synuclein protofibrils are produced when spherical protofibrils are incubated in solution or bound to brain-derived membranes. Biochemistry 41, 10209-10217. 
(22) Cappai, R., Leck, S. L., Tew, D. J., Williamson, N. A., Smith, D. P., Galatis, D., Sharples, R. A., Curtain, C. C., Ali, F. E., Cherny, R. A., Culvenor, J. G., Bottomley, S. P., Masters, C. L., Barnham, K. J., and Hill, A. F. (2005) Dopamine promotes alphasynuclein aggregation into SDS-resistant soluble oligomers via a distinct folding pathway. FASEB J. 19, 1377.

(23) Sharon, R., Bar-Joseph, I., Frosch, M. P., Walsh, D. M., Hamilton, J. A., and Selkoe, D. J. (2003) The formation of highly soluble oligomers of alpha-synuclein is regulated by fatty acids and enhanced in Parkinson's disease. Neuron 37, 583-595.

(24) Segers-Nolten, I. M. J., Wilhelmus, M. M. M., Veldhuis, G., van Rooijen, B. D., Drukarch, B., and Subramaniam, V. (2008) Tissue transglutaminase modulates alpha-synuclein oligomerization. Protein Sci. 17, 1395-1402.

(25) Kayed, R., Head, E., Thompson, J. L., McIntire, T. M., Milton, S. C., Cotman, C. W., and Glabe, C. G. (2003) Common structure of soluble amyloid oligomers implies common mechanism of pathogenesis. Science 300, 486-489.

(26) Lindgren, M., Sörgjerd, K., and Hammarström, P. (2005) Detection and characterization of aggregates, prefibrillar amyloidogenic oligomers, and protofibrils using fluorescence spectroscopy. Biophys. J. 88, 4200-4212.

(27) Hawe, A., Sutter, M., and Jiskoot, W. (2008) Extrinsic fluorescent dyes as tools for protein characterization. Pharmaceut. Res. 25, 1487-1499.

(28) Semisotnov, G. V., Rodionova, N. A., Razgulyaev, O. I., Uversky, V. N., Gripas, A. F., and Gilmanshin, R. I. (1991) Study of the molten globule intermediate state in protein folding by a hydrophobic fluorescent-probe. Biopolymers 31, 119-128.

(29) Krebs, M. R. H., Bromley, E. H. C., and Donald, A. M. (2005) The binding of thioflavin- $\mathrm{T}$ to amyloid fibrils: localisation and implications. J. Struct. Biol. 149, 30-37.

(30) Diwu, Z., Lu, Y. X., Zhang, C. L., Klaubert, D. H., and Haugland, R. P. (1997) Fluorescent molecular probes.2. The synthesis, spectral properties and use of fluorescent solvatochromic Dapoxyl dyes. Photochem. Photobiol. 66, 424-431.

(31) Thumser, A. E. A., Voysey, J. E., and Wilton, D. C. (1994) The Binding Of Lysophospholipids To Rat-Liver Fatty-Acid-Binding Protein And Albumin. Biochem. J. 301, 801-806. 
Oligomeric $\alpha$-synuclein and its role in aggregation

(32) Sackett, D. L., and Wolff, J. (1987) Nile Red As A PolaritySensitive Fluorescent-Probe Of Hydrophobic Protein Surfaces. Anal. Biochem. 167, 228-234.

(33) Nitsche, J. M., Chang, H. C., Weber, P. A., and Nicholson, B. J. (2004) A transient diffusion model yields unitary gap junctional permeabilities from images of cell-to-cell fluorescent dye transfer between Xenopus oocytes. Biophys. J. 86, 2058-2077.

(34) Pristinski, D., Kozlovskaya, V., and Sukhishvili, S. A. (2005) Fluorescence correlation spectroscopy studies of diffusion of a weak polyelectrolyte in aqueous solutions. J. Chem. Phys. 122.

(35) Quist, A., Doudevski, I., Lin, H., Azimova, R., Ng, D., Frangione, B., Kagan, B., Ghiso, J., and Lal, R. (2005) Amyloid ion channels: a common structural link for protein-misfolding disease. Proc. Natl. Acad. Sci. U. S. A. 102, 10427-10432.

(36) Celej, M. S., Jares-Erijman, E. A., and Jovin, T. M. (2008) Fluorescent $\mathrm{N}$-arylaminonaphthalene sulfonate probes for amyloid aggregation of alpha-synuclein. Biophys. J. 94, 4867-4879.

(37) Christensen, H., and Pain, R. H. (1991) Molten Globule Intermediates And Protein Folding. Eur. Biophys. J. 19, 221-229.

(38) Mach, H., and Middaugh, C. R. (1995) Interaction Of Partially Structured States Of Acidic Fibroblast Growth-Factor With Phospholipid-Membranes. Biochemistry 34, 9913-9920.

(39) Halskau, O., Underhaug, J., Frøystein, N. A., and Martínez, A. (2005) Conformational flexibility of alpha-lactalbumin related to its membrane binding capacity. J. Mol. Biol. 349, 1072-1086.

(40) Song, M. S., Shao, H. Y., Mujeeb, A., James, M. L., and Miller, W. L. (2001) Molten-globule structure and membrane binding of the N-terminal protease-resistant domain (63-193) of the steroidogenic acute regulatory protein (StAR). Biochem. J. 356, 151-158.

(41) Bañuelos, S., and Muga, A. (1995) Binding Of Molten GlobuleLike Conformations To Lipid Bilayers - Structure Of Native And Partially Folded Alpha-Lactalbumin Bound To Model Membranes. J. Biol. Chem. 270, 29910-29915.

(42) Hess, S. T., and Webb, W. W. (2003) Focal volume optics and experimental artifacts in confocal fluorescence correlation spectroscopy. Biophys. J. 84, 313A-313A.

(43) Morar, A. S., Olteanu, A., Young, G. B., and Pielak, G. J. (2001) Solvent-induced collapse of alpha-synuclein and acid-denatured cytochrome c. Protein Sci. 10, 2195-2199.

(44) Uversky, V. N. (2002) What does it mean to be natively unfolded? Eur. J. Biochem. 269, 2-12. 
(45) Hoyer, W., Antony, T., Cherny, D., Heim, G., Jovin, T. M., and Subramaniam, V. (2002) Dependence of alpha-synuclein aggregate morphology on solution conditions. J. Mol. Biol. 322, 383-393.

(46) Leach, S. J., and Scheraga, H. A. (1960) Effect of Light Scattering on Ultraviolet Difference Spectra. J. Am. Chem. Soc. 82, 47904792 .

(47) van der Werf, K. O., Putman, C. A. J., de Grooth, B. G., Segerink, F. B., Schipper, E. H., van Hulst, N. F., and Greve, J. (1993) Compact Stand-Alone Atomic-Force Microscope. Rev. Sci. Instrum. 64, 2892-2897. 


\section{Chapter 3 Lipid bilayer disruption by oligomeric $\alpha$-synuclein}

Soluble oligomeric aggregates of $\alpha$-synuclein have been implicated to play a central role in the pathogenesis of Parkinson's disease. Disruption and permeabilization of lipid bilayers by oligomeric $\alpha$-synuclein is postulated as a toxic mechanism, but the molecular details controlling the oligomer-membrane interaction are still unknown. Here we show that membrane disruption strongly depends on the accessibility of the hydrophobic membrane core and that charge interactions play an important but complex role. We systematically studied the influence of the physical membrane properties and solution conditions on lipid bilayer disruption by oligomers using a dye release assay. Varying the lipid headgroup composition revealed that membrane disruption only occurs for negatively charged bilayers. Furthermore, vesicle disruption is inhibited at low ionic strength. The disruption of negatively charged vesicles further depends on lipid packing parameters. Bilayer composition changes that result in an increased lipid headgroup spacing make vesicles more prone to disruption, suggesting that the accessibility of the bilayer hydrocarbon core modulates oligomer-membrane interaction. These data shed insights into the driving forces governing the highly debated process of oligomer-membrane interactions. 


\section{Introduction}

Oligomeric intermediates in the aggregation process of $\alpha \mathrm{S}$ have been hypothesized to be the toxic species. A proposed mechanism of toxicity is lipid membrane disruption $(1,2)$. An increased permeability of the cellular membrane could lead to neurodegeneration via an altered calcium homeostasis (3). In vitro it has been shown that $\alpha \mathrm{S}$ oligomers can disrupt phospholipid vesicles (4). The proposed disruption mechanism is the formation of pore-like structures within the lipid bilayer, although other mechanisms such as bilayer thinning have been proposed (5). This hypothesis has been supported by single channel recording $(6,7)$, a selectivity for the marker size in an efflux assay (8) and the observation of donut shaped complexes by atomic force microcopy and electron microscopy $(9,10)$. However, the mechanism of $\alpha \mathrm{S}$ membrane disruption and how it relates to $\mathrm{PD}$ is still a hotly debated subject.

To correctly identify the mechanism through which membrane permeabilization is facilitated, it is crucial to understand the interaction between the oligomers and lipids. However, very little is known about what factors determine this interaction. To address this issue we comprehensively studied the membrane disruptive effect of $\alpha \mathrm{S}$ oligomers using a dye release assay on lipid vesicles. By systematically altering the vesicle lipid composition and solution conditions we mapped the fundamental driving forces governing the oligomerlipid interaction. We show that membrane permeabilization is mainly determined by the presence of negatively charged lipids in the bilayer. At low ionic strengths, vesicle disruption was inhibited, thereby suggesting a complex role for electrostatic interactions. Furthermore, both the headgroup specificity of the interaction and the dependence on the bilayer packing parameters imply that disruption occurs through interaction with the bilayer core.

\section{Results}

\section{Membrane Permeabilization}

Membrane disruption by $\alpha \mathrm{S}$ was characterized using a dye efflux assay. Large unilamellar vesicles (LUVs) were filled with $50 \mathrm{mM}$ calcein, a concentration at which calcein fluorescence self-quenches. Upon leakage from the vesicle, quenching of the dye is relieved and a fluorescence signal is observed. The intensity after 30 minutes of incubation was measured. The kinetics of dye leakage induced by oligomeric $\alpha \mathrm{S}$ are fast, making this essentially an endpoint measurement (Figure 1A). The extent of dye leakage from POPG LUVs was clearly dependent on the protein concentration for all aggregation states (Figure 1B). Oligomeric $\alpha \mathrm{S}$ was most effective in disrupting the LUVs and induced a 50 $\%$ leakage of the LUVs at a concentration of $\sim 0.13 \mu \mathrm{M}$. Both the monomeric 

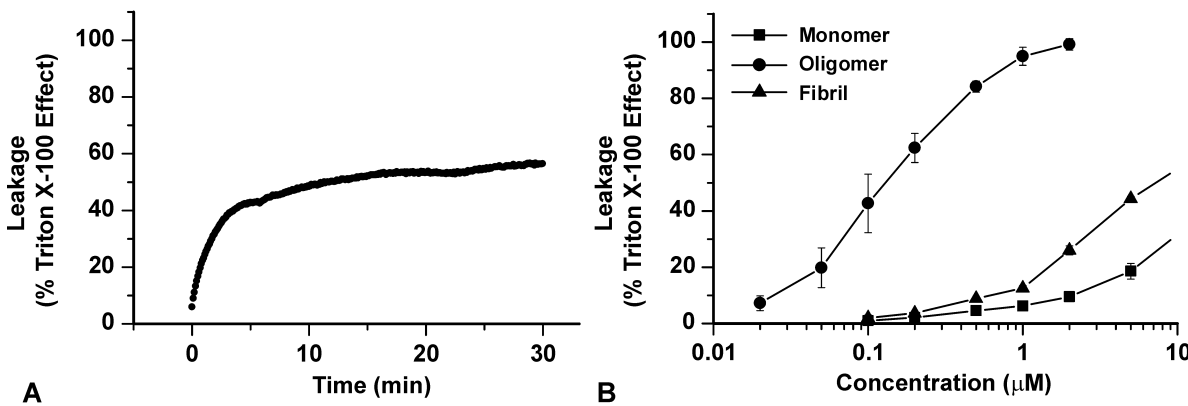

Figure 1. Vesicle disruption by $\alpha \mathbf{S}$ (A) Kinetics of calcein efflux from $20 \mu \mathrm{M}$ POPG LUVs as induced by $0.015 \mu \mathrm{M}$ oligomeric $\alpha \mathrm{S}$. The leakage kinetics contains an initial fast phase, after which leakage gradually continues. (B) Dependence of the content leakage from POPG LUVs on the concentration of $\alpha \mathrm{S}$ monomers, oligomers and fibrils at a phospholipid concentration of $20 \mu \mathrm{M}$. Leakage is expressed as a percentage of the maximum possible effect induced by the addition of triton X-100. The protein concentration for the oligomeric and fibrillar species is given as the equivalent monomer concentration. The error bars indicate the standard deviation $(n=3)$.

and fibrillar form of the protein were also able to disrupt the LUVs, although at significantly higher protein concentrations. These induced $50 \%$ vesicle leakage at $\sim 21 \mu \mathrm{M}$ and $\sim 7.0 \mu \mathrm{M}$ respectively. The disease related mutants $\alpha \mathrm{S}-\mathrm{A} 30 \mathrm{P}$, $\alpha \mathrm{S}-\mathrm{E} 46 \mathrm{~K}$ and $\alpha \mathrm{S}-\mathrm{A} 53 \mathrm{~T}$ showed similar disruptive properties (Figure 2).

\section{Lipid headgroup Specificity}

It is not known which properties of the lipid bilayer determine the interaction with the oligomeric intermediates. Membrane permeabilization by $\alpha \mathrm{S}$ oligomers has been reported to depend on lipid headgroup composition $(8,11)$, but the data are contradictory. To assess the headgroup specificity for membrane disruption, we prepared LUVs composed of phospholipids with different headgroups. The results from these experiments clearly show that only vesicles composed of negatively charged phospholipids show dye efflux upon addition of $1 \mu \mathrm{M}$ of oligomeric $\alpha \mathrm{S}$ (Figure 3) (The chemical structure of the lipids are shown in Appendix B). POPG, PI and DOPS all showed calcein efflux upon addition of the oligomeric species. LUVs from the zwitterionic POPC were not disrupted by $\alpha \mathrm{S}$. This suggests that charge interactions play an important role in oligomer-lipid interaction. Mixing zwitterionic lipids, such as POPC and DOPE, with anionic lipids in a 1:1 molar ratio, inhibited the vesicle disruption for POPG, DOPS and PI containing vesicles. Vesicle preparations from the anionic lipids DOPA and CL were rather unstable. Therefore, to probe 


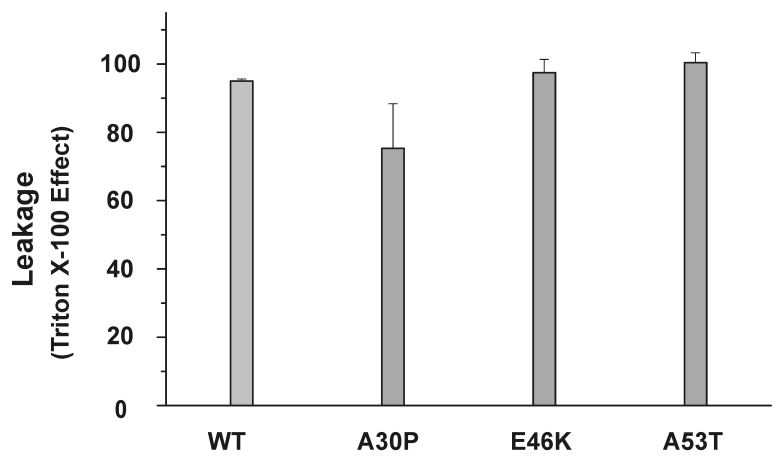

Figure 2. Mutant $\alpha$ S. Calcein efflux from $20 \mu \mathrm{M}$ POPG LUVs induced by $1 \mu \mathrm{M}$ oligomeric $\alpha \mathrm{S}$ from $\alpha \mathrm{S}$-wt and the disease related mutants $\alpha \mathrm{S}-\mathrm{A} 30 \mathrm{P}, \alpha \mathrm{S}-\mathrm{E} 46 \mathrm{~K}$ and $\alpha$ S-A53T. Oligomers from all mutants were able to disrupt the POPG LUVs. The error bars indicate the standard deviation $(n=3)$.

the interaction of oligomeric $\alpha \mathrm{S}$ with these lipids, vesicles were prepared from 1:1 mixtures of anionic CL and DOPA with zwitterionic POPC. These vesicles showed considerable dye release. Monomeric and fibrillar $\alpha \mathrm{S}$ were also added to the LUVs at a ten times higher concentration. These induced some dye efflux from POPG LUVs and from PI LUVs. The amount of efflux was still smaller than the efflux induced by $1 \mu \mathrm{M}$ oligomeric $\alpha \mathrm{S}$.

Compared to other negatively charged lipids, DOPA was most sensitive to disruption by oligomeric $\alpha \mathrm{S}$, as 1 to 1 molar mixtures with POPC were still vulnerable to disruption by oligomeric $\alpha \mathrm{S}$. In order to observe at what charge density DOPC:DOPA mixtures are permeabilized by $\alpha$ S oligomers, LUVs from different ratios of DOPC to DOPA were prepared. As can be seen in Figure 4A, a high amount of negatively charged lipids in the membrane is needed before oligomer induced vesicle disruption occurs. When DOPC and DOPA are mixed in a 2:1 or 3:1 ratio, disruption is inhibited even at high protein concentrations.

To further investigate the role of charge interactions in $\alpha \mathrm{S}$ induced membrane disruption, a dye release experiment at different ionic strengths was performed (Figure 4B). This revealed very clearly that at low ionic strength $(0$ and $5 \mathrm{mM}$ added $\mathrm{NaCl}$ ) vesicle disruption was strongly inhibited. At higher salt concentrations of $50 \mathrm{mM}$ and $150 \mathrm{mM} \mathrm{NaCl}$ a clear concentration dependent calcein efflux was observed. 


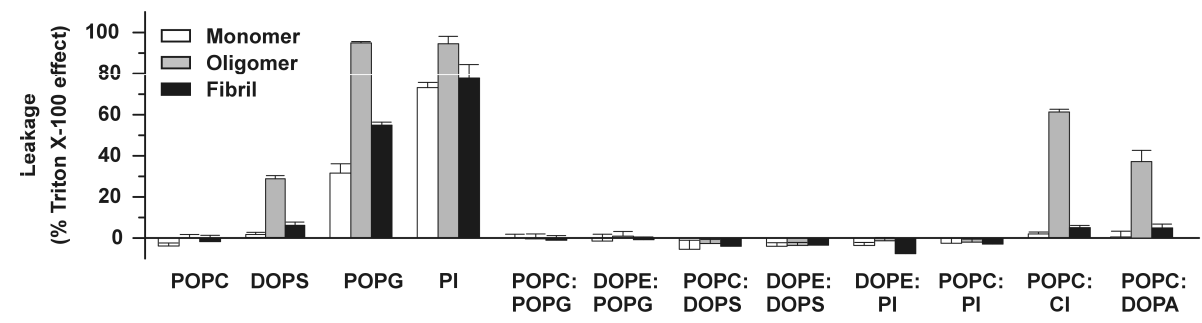

Figure 3. Lipid headgroup composition. Calcein efflux from LUVs of different lipid compositions induced by $10 \mu \mathrm{M}$ monomeric, $1 \mu \mathrm{M}$ oligomeric and $10 \mu \mathrm{M}$ fibrillar $\alpha \mathrm{S}$ at a phospholipid concentration of $20 \mu \mathrm{M}$. Lipid mixtures were prepared in a 1:1 molar ratio. The error bars indicate the standard deviation $(n=3)$.

\section{Lipid packing parameters}

The increased sensitivity of DOPA and CL to oligomeric $\alpha \mathrm{S}$ might be related to their small headgroup size. Thus the accessibility of the hydrocarbon bilayer core could be important in determining the lipid interaction of oligomeric $\alpha \mathrm{S}$. Moreover, small unilamellar vesicles $(\sim 30 \mathrm{~nm})$ have been shown to be more vulnerable to disruption by the oligomeric species (4). Curvature stress within the bilayer, which can lead to packing defects, is a possible explanation for such an effect. Although the exact nature of such defects is not clear, they might allow proteins to more easily interact with the hydrophobic core of the membrane (12). These data indicate that lipid packing parameters could modulate oligomer-lipid interactions. Therefore the effect of lipid packing properties on the disruption of vesicles by $\alpha \mathrm{S}$ oligomers was investigated.

First, lipid packing was influenced by altering the spontaneous curvature of the phospholipids in the bilayer, which can have a large effect on lipid protein interaction $(12,13)$. The spontaneous curvature of a lipid monolayer is a result of the shape of the lipids (14). Lipids with a small headgroup have a negative radius of curvature, whereas lipids with a single acyl chain have a positive radius of curvature (12). DOPA, CL and POPG in high salt concentrations are expected to have negative spontaneous curvature (15). However, in LUVs the lipids are packed in an almost planar bilayer. Thus in vesicles composed of these lipids the hydrophobic lipid bilayer core might be more exposed to the oligomers. The spontaneous curvature can be modulated towards a more positive value without changing the headgroup compositions by adding lyso lipids. These have a single acyl chain (15) and thus a positive radius of spontaneous curvature. Adding such lyso lipids to vesicles containing packing defects due to curvature stress, lipid packing of the LUVs may be optimized, relieving the curvature stress in the membrane. 

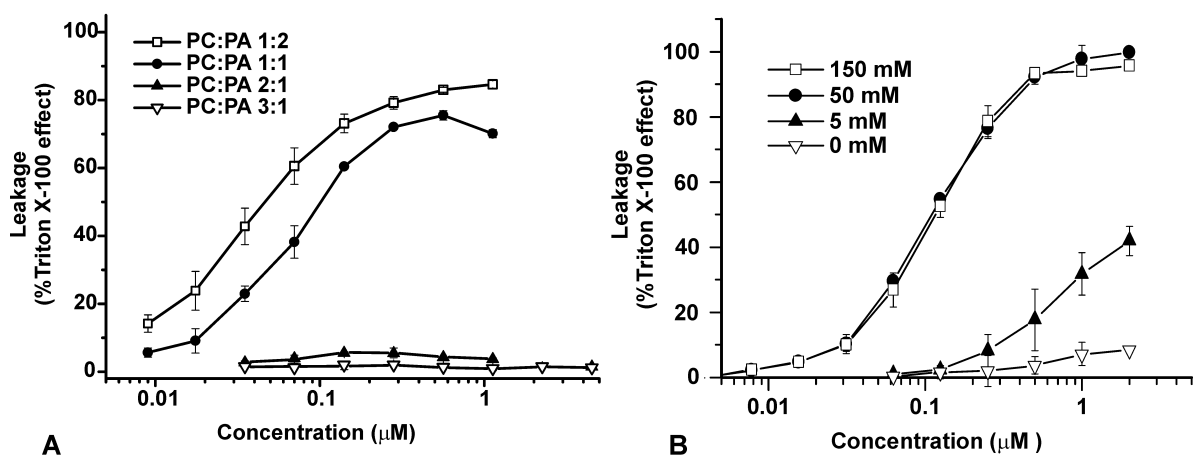

Figure 4. Charge interactions (A) Calcein efflux from LUVs of different lipid compositions induced by $1 \mu \mathrm{M}$ oligomeric $\alpha \mathrm{S}$ at a phospholipid concentration of 20 $\mu \mathrm{M}$. LUVs were prepared from different ratios of DOPC to DOPA: 1:2, 1:1, 2:1 and 3:1. The error bars indicate the standard deviation $(n=2)$. (B) The effect of the ionic strength of the solution on the disruption of POPG LUVs measured by calcein efflux at a phospholipid concentration of $20 \mu \mathrm{M}$. $\alpha$ S oligomers were added at different concentrations to the vesicle solution in the presence of $0 \mathrm{mM}, 5 \mathrm{mM}, 50 \mathrm{mM}$ and $150 \mathrm{mM} \mathrm{NaCl}$. The error bars indicate the standard deviation $(n=3)$.

Lyso PG was added at different ratios to POPG from which LUVs were subsequently obtained by extrusion. Membrane permeability induced by the addition of $\alpha \mathrm{S}$ oligomers was measured at different protein concentrations. The results are shown in Figure 5A. Pure POPG LUVs showed a $50 \%$ leakage at a $\alpha \mathrm{S}$ concentration of $0.25 \mu \mathrm{M}$. Optimizing the lipid bilayer packing by the addition of increasing amounts of lyso PG stabilized the vesicles against permeabilization by $\alpha \mathrm{S}$, with $50 \%$ leakage at $0.42 \mu \mathrm{M} \alpha \mathrm{S}$ for $10 \%$ lyso PG and at $0.79 \mu \mathrm{M} \alpha \mathrm{S}$ for $20 \%$ lyso PG containing vesicles. This result indicates that lipid packing of the LUVs, and thus the accessibility of the membrane core, is an important factor in oligomer-lipid interaction.

Lipid packing is also affected by changing the amount of unsaturated bonds in the lipid hydrocarbon chains. Upon increasing unsaturation, chain disorder increases and the average headgroup spacing becomes larger, resulting in a thinner bilayer (16). To probe the effects of lipid chain packing, LUVs of lipids with phosphatidylglycerol headgroups containing different acyl chain configurations were produced. Fully saturated lipids were not probed because of the high gel to fluid phase transition temperature of these lipids. Cholesterol is a well known modulator of lipid bilayer packing and enhances the cohesive interactions within the bilayer which results in a more densely packed bilayer (16). Therefore, POPG LUVs containing $25 \%$ cholesterol were also prepared. 

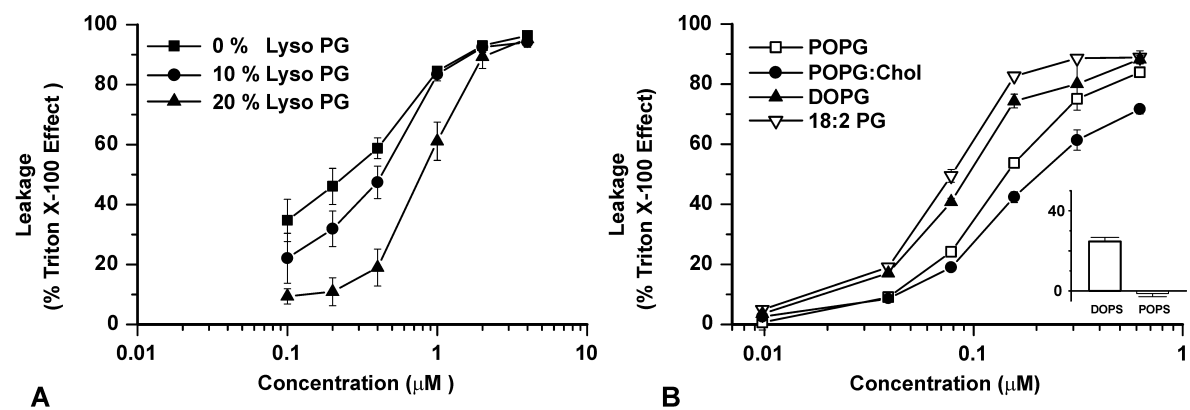

Figure 5. Lipid packing (A) The effect of headgroup spacing on oligomer-induced vesicle leakage. LUVs were prepared from POPG with different amounts of lyso PG. Total lipid concentration was $25 \mu \mathrm{M}$ with vesicles containing $0 \%, 10 \%$ and $20 \%$ lyso PG. (B) Influence of lipid packing parameters on oligomer induced vesicle disruption. Plot of the concentration dependent oligomer-induced dye release from $20 \mu \mathrm{M}$ PG LUVs with different acyl chains: POPG, DOPG, 18:2 PG and POPG:Cholesterol $3: 1$. The error bars indicate the standard deviation $(n=3)$. The inset shows calcein efflux from DOPS and POPS LUVs at a lipid concentration of $20 \mu \mathrm{M}$. Oligomer concentration was $1 \mu \mathrm{M}$ for DOPS LUVs and $10 \mu \mathrm{M}$ for the POPS LUVs.

The effect of $\alpha \mathrm{S}$ on the permeability of these different LUVs was measured at different oligomer concentrations as shown in Figure 5B. The results reveal that upon increasing saturation the vesicles are more stable against $\alpha \mathrm{S}$ induced permeabilization. The amount of dye release from the different lipid compositions was most efficient in 18:2 PG and decreased upon increasing chain saturation. The addition of $25 \%$ cholesterol to POPG LUVs further stabilized these vesicles. The oligomer concentrations at which $50 \%$ leakage is induced are as follows: $18: 2 \mathrm{PG} \sim 0.08 \mu \mathrm{M}, \mathrm{DOPG} \sim 0.1 \mu \mathrm{M}, \mathrm{POPG} \sim 0.15 \mu \mathrm{M}$ and POPG:Chol $\sim 0.2 \mu \mathrm{M}$. These data confirm that an increase in lipid headgroup spacing facilitates the interaction of the oligomer with the membrane. We observed a similar effect of lipid packing for vesicles composed of lipids with a phosphatidylserine headgroup. DOPS LUVs could be disrupted by oligomeric $\alpha \mathrm{S}$, whereas in LUVs from POPS calcein efflux was inhibited even at high protein concentration. The complete inhibition of calcein efflux from POPS LUVs might be related to the fact that the phase transition temperature of this lipid is close to room temperature. 

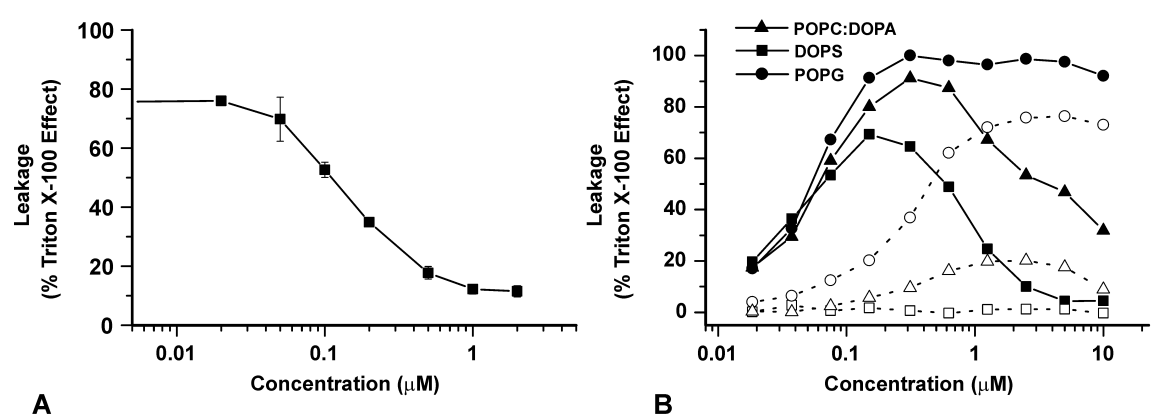

Figure 6. The effect of monomeric $\alpha \mathrm{S}$ on oligomer-induced vesicle disruption (A) Calcein efflux experiment on $25 \mu \mathrm{M}$ POPG LUVs. The LUVs were pre-incubated for 30 minutes with different concentrations of monomeric $\alpha \mathrm{S}$. Oligomeric $\alpha \mathrm{S}$ was subsequently added to a final concentration of $0.5 \mu \mathrm{M}$. Efflux was measured after another 30 minute incubation. The error bars indicate the standard deviation $(n=3)$. (B) Calcein efflux from POPG, DOPS and POPC:DOPA LUVs (20 $\mu \mathrm{M}$ total phospholipid) induced by different concentrations of oligomeric $\alpha \mathrm{S}$ either in the presence (open symbols) or absence (closed symbols) of $0.2 \mu \mathrm{M}$ monomeric $\alpha \mathrm{S}$.

Monomeric $\alpha \mathrm{S}$ has been reported to optimize lipid packing in small vesicles (17). Correspondingly, preincubating LUVs with monomeric $\alpha \mathrm{S}$ can inhibit the membrane permeabilization by oligomeric $\alpha \mathrm{S}$ (Figure 6A). This effect was dependent on the amount of monomer added. The effect was also dependent on the lipid composition used and was strongest in LUVs composed of DOPS (Figure 6B). Such an effect could also explain a maximum that was observed in the leakage curves for DOPS and POPC:DOPA LUVs. A residual amount of monomeric $\alpha \mathrm{S}$ in stock solutions of the oligomeric species might stabilize the vesicles at high amounts of added oligomers.

\section{Discussion}

Membrane disruption by $\alpha \mathrm{S}$ oligomers is often considered as a likely mechanism of cytotoxicity in PD. However, due to the lack of understanding of the fundamental molecular mechanisms involved, it is difficult to estimate the biological relevance of this process. In this chapter we have used a dye release assay to screen a range of conditions that could modulate oligomer-lipid interaction. By studying how physicochemical bilayer properties and solution conditions influence $\alpha \mathrm{S}$ oligomer-induced vesicle disruption fundamental driving forces governing oligomer-lipid interactions have been identified. 
Lipid bilayer disruption by oligomeric $\alpha$-synuclein

\section{Vesicle Permeabilization}

Our experiments clearly show that $\alpha \mathrm{S}$ oligomers can permeabilize POPG LUVs in a concentration dependent fashion. Using similar conditions, Volles et al. (8) found leakage at around 30 times lower protein to lipid ratios. Most likely, the difference in size of the markers used in the different assays used; calcein efflux versus calcium influx, can influence the amount of observed leakage. Additionally, the divalent calcium cation can have a profound effect on negatively charged vesicles in general and on $\alpha$ S-lipid interaction specifically $(18,19)$.

At very high protein concentrations monomeric and fibrillar forms of $\alpha \mathrm{S}$ were also able to induce calcein leakage from POPG LUVs. Monomeric $\alpha \mathrm{S}$ is thought to disrupt LUVs in a detergent-like way. How fibrillar $\alpha \mathrm{S}$ can cause membrane damage is not known; apparently fibrillar $\alpha \mathrm{S}$ is able to bind to a lipid bilayer. We however cannot exclude the possibility that the monomeric protein forms aggregates on the lipid membranes or that the fibrils dissociate into oligomers. Oligomerization of monomeric $\alpha \mathrm{S}$ on the membrane surface has been suggested by MD simulation (20) and aggregation experiments (21), while single channel activity has been observed upon addition of the monomeric protein (22).

$\alpha \mathrm{S}$ oligomers from the disease related mutants A30P, E46K and A53T showed very similar leakage characteristics. Although no major quantitative difference in induced leakage was observed between the different mutants, oligomers derived from $\mathrm{A} 30 \mathrm{P} \alpha \mathrm{S}$ appeared to induce the least vesicle disruption. Interestingly, monomeric $\mathrm{A} 30 \mathrm{P} \alpha \mathrm{S}$ is also reported to bind lipids with a lower affinity than wild type $\alpha \mathrm{S}$ and the E46K and A53T mutants $(23,24)$.

\section{Driving forces}

Our results indicate that only negatively charged vesicles are affected by oligomeric $\alpha \mathrm{S}$. Therefore, charge interactions appear to determine oligomer mediated membrane disruption. Although monomeric $\alpha \mathrm{S}$ is highly negatively charged, it specifically binds to negatively charged membranes $(25,26)$. This binding is explained by the fact that at neutral $\mathrm{pH}$ the N-terminal lipid binding region contains many positively charged residues. Furthermore, most of the negative charge is concentrated in the $\mathrm{C}$-terminus of the protein, which remains unstructured in solution upon protein-lipid binding (27). Although the structure of oligomeric $\alpha \mathrm{S}$ in its free- and lipid-bound state is not known, a clear charge separation as in the monomeric protein seems unlikely to be energetically favorable. However, specific binding of oligomeric $\alpha \mathrm{S}$ to negatively charged vesicles has been reported (8).

In addition, the role of charge interactions in oligomer membrane disruption appears to be more complex. Calcein efflux experiments at different ionic strengths showed that leakage is inhibited at low salt concentrations. For bind- 
ing mediated by charge interaction one would expect to observe stronger binding and thus leakage at lower protein concentrations. Possibly, reduced screening of electrostatic interactions at low ionic strength between the negatively charged oligomers and the bilayer surface causes an electrostatic repulsion inhibiting binding. Positively charged or hydrophobic patches on the surface of the oligomer might support the oligomer binding.

However, there are many contributing factors making it difficult to pinpoint the exact binding mechanism. For instance, the structure of the $\alpha \mathrm{S}$ oligomers might change as an effect of ionic strength. Finally, lipid packing is also influenced by the ionic strength, further contributing to the complexity of the system. In addition to bilayer charge, lipid packing parameters also influence the oligomer-induced vesicle disruption. We observed that more densely packed bilayers were more resistant to disruption. This suggests that hydrophobic interactions contribute to oligomer-lipid binding. The membrane interior of loosely packed bilayers is much more accessible. Interaction with the membrane hydrocarbon core might facilitate binding and disruption.

\section{Mechanism of disruption}

One of the key questions in oligomer-lipid interactions revolves around the mechanism by which bilayer disruption occurs. The dependence of membrane disruption on the bilayer physical properties can give important information on the most likely mechanism of membrane disruption (28). Besides a pore-like mechanism $(6,8)$, membrane thinning has been suggested as a possible mode of action $(5,29)$. Adsorption of the oligomeric intermediates to the surface of the bilayer could cause an increase in the average headgroup spacing and thus have a thinning effect. Bilayer thinning increases the membrane permeability by decreasing the thickness of the hydrophobic barrier. Lipid bilayers that are more easily compressed are expected to show greater membrane permeability. Addition of cholesterol to the bilayer which reduces the compressibility of the bilayer did show a slight inhibitory effect. Our results suggest that a thinner membrane with increased headgroup spacing facilitates the leakage process. However, a thinner bilayer may be the cause of oligomer-lipid interaction and not necessarily the effect. Pore formation through oligomer insertion into the bilayer core still offers a plausible explanation for all the observed effects.

\section{Biological significance}

The oligomers did not show a toxic effect upon the addition to the medium of cultured cells. However this may not be the most relevant assay since $\alpha \mathrm{S}$ is an intracellular protein. Toxic effects have been observed upon extracellular addition of oligomeric $\alpha \mathrm{S}$ prepared using a different protocol $(30,31)$. Possibly there are functionally distinct oligomeric species. From the observed lipid speci- 
Lipid bilayer disruption by oligomeric $\alpha$-synuclein

ficity one could also predict likely cellular targets for $\alpha \mathrm{S}$ oligomer membrane disruption. The plasma membrane is rich in sterols, spingholipids and phosphatidylcholines (32). Our data show that POPC and cholesterol both inhibit leakage therefore the plasma membrane is not a likely target for oligomeric $\alpha \mathrm{S}$. Although $\alpha \mathrm{S}$ in its monomeric and aggregated form have been observed in the extracellular space and can be possibly secreted or released from dying cells (33), the majority of the protein is intracytoplasmic. Therefore the membranes of organelles are a possible target of $\alpha \mathrm{S}$ oligomers.

In this light the observed leakage specificity for the mitochondrial lipid cardiolipin suggests that mitochondria could be vulnerable to $\alpha \mathrm{S}$ oligomers. Mitochondrial dysfunction has long been suggested to play a potential role in the pathogenesis of $\mathrm{PD}(34)$. $\alpha \mathrm{S}$ has been observed to localize to mitochondria (35), which is increased upon $\alpha \mathrm{S}$ overexpression (36) and intracellular acidification (37). Mitochondrial localization of $\alpha \mathrm{S}$ is possibly mediated by cardiolipin since monomeric $\alpha \mathrm{S}$ has been reported to bind cardiolipin with high affinity (38) in vitro. Possibly, $\alpha \mathrm{S}$ oligomers directly induce mitochondrial membrane damage. Additionally interactions with mitochondrial proteins, such as mitochondrial complex I could also be a source of cell damage (39-41). However, it should be pointed out that membrane disruption was only observed in membranes which have high anionic lipid content. At physiologically more relevant concentrations, such as POPC:Cl 3:1 membrane disruption was inhibited. How these results translate to the effect of $\alpha \mathrm{S}$ oligomers in vivo is unclear, since real biological membranes are much more heterogeneous and contain distinct domains that may mediate oligomer-membrane interactions.

Summarizing, the disruption of LUVs by oligomeric $\alpha \mathrm{S}$ is primarily dependent on the charge of the membrane. Only negatively charged membranes are affected and mixing negatively charged lipids with zwitterionic lipid generally inhibits the leakage process. Bilayer packing affects oligomer-membrane disruption to a smaller extent. More densely packed membranes appear to be less prone to disruption by $\alpha \mathrm{S}$ oligomers.

\section{Materials and Methods}

Expression and purification of recombinant $\alpha S$. Expression of the human $\alpha \mathrm{S}$-wt and the disease related mutant proteins A30P, E46K and A53T were performed as described in Chapter 2.

Preparation of $\alpha S$ oligomeric and fibrillar species. $\alpha \mathrm{S}$ oligomers were prepared as described in Chapter 2. Mature $\alpha \mathrm{S}$ fibrils were prepared by incubating the stock protein solution in a thermo mixer for 10 days at $37^{\circ} \mathrm{C}$ at $1250 \mathrm{rpm}$. The aggregated protein was pelleted by centrifugation for 40 minutes at 21,000 $\times \mathrm{g}$. The pellet was washed and resuspended twice in $10 \mathrm{mM}$ HEPES, $\mathrm{pH}$ 7.4, 
$150 \mathrm{mM} \mathrm{NaCl}$. To estimate the protein concentration in the fibril containing solution, the aggregates were dissolved in $5 \mathrm{M}$ guanidine hydrochloride, and the protein concentration was calculated from the absorption measured at 275 nm.

Liposome preparation. 1-Palmitoyl, 2-oleoyl phosphatidylcholine (POPC), 1,2-dioleoyl phosphatidylcholine (DOPC), 1-palmitoyl, 2-oleoyl phosphatidylglycerol (POPG), 1,2-dioleoyl phosphatidylglycerol (DOPG), 1-oleoyl phosphatidylglycerol (lyso PG), 1,2-dilinoleoyl phosphatidylglycerol (18:2 PG), 1palmitoyl, 2-oleoyl phosphatidylserine (POPS), 1,2-dioleoyl phosphatidylserine (DOPS), 1,2-dioleoyl phosphatidic acid (DOPA), 1,2-dioleoyl phosphatidylethanolamine (DOPE), soy L- $\alpha$-phosphatidylinositol (PI), bovine heart cardiolipin (CL) and cholesterol (chol) were all obtained from Avanti Polar lipids and used without further purification. The various lipid compositions were prepared by mixing the chloroform dissolved lipids to specific ratios. Lipid acyl chain compositions were chosen to minimize the chance of phase separation in the mixtures. To prepare LUVs, a thin lipid film was formed by drying around $0.5 \mathrm{mg}$ of lipid in a glass tube using a gentle stream of nitrogen gas. Trace amounts of solvent were removed by drying under vacuum for 4 hours. The lipid film was then hydrated by adding a solution of $50 \mathrm{mM}$ calcein (Sigma), 10mM HEPES, pH 7.4. Appropriate amounts of $\mathrm{NaCl}$ were added to maintain an osmotic strength equal to $10 \mathrm{mM}$ HEPES $\mathrm{pH} 7.4,150 \mathrm{mM}$ $\mathrm{NaCl}$. Hydration was continued for 1 hour, with vortexing approximately every 15 minutes. The sample was subsequently subjected to 5 freeze-thaw cycles by dipping into liquid nitrogen and thawing above the lipid phase transition temperature. The resulting solution was extruded 11 times through a polycarbonate membrane filter with a $100 \mathrm{~nm}$ pore size. This procedure was repeated once with a new filter. Unencapsulated dye was separated from the vesicles by gel filtration through a PD10 column packed with Sephadex G-100 (GE). The vesicle size distribution was measured using dynamic light scattering on a Malvern Zetasizer 4000 instrument. The total phospholipid concentration was determined according to the protocol of Chen et al. (42).

Efflux assay. For the efflux assay, the stock vesicle solution was first diluted to twice the final phospholipid concentration and then thoroughly mixed with an equal volume of protein solution. After 30 minutes incubation the maximum intensity of the fluorescence emission spectrum for excitation at $497 \mathrm{~nm}$ was recorded on a Varian Cary Eclipse fluorimeter. After subtraction of the background, leakage was expressed as a percentage of the maximum possible effect due to vesicle disruption induced by the addition of $0.5 \%$ (weight/volume) Triton X-100. Experiments aiming to elucidate the influence of a certain single parameter were performed on the same preparation of oligomers, to negate possible batch to batch differences in oligomer preparation and potential errors 
Lipid bilayer disruption by oligomeric $\alpha$-synuclein

in the concentration determination.

Ionic strength assay. For the ionic strength experiment, solutions of 10 mM HEPES pH 7.4 containing $0 \mathrm{mM}, 5 \mathrm{mM}$ or $50 \mathrm{mM} \mathrm{NaCl}$ were prepared. For these salt concentrations lipids were hydrated in $50 \mathrm{mM}$ calcein, $10 \mathrm{mM}$ HEPES pH 7.4. The $\mathrm{NaCl}$ buffer solutions were adjusted with sucrose to equal osmolarity as the vesicle hydration solution. Vesicles for the $\mathrm{NaCl}$ concentration of $150 \mathrm{mM}$ were prepared as described earlier. $\alpha \mathrm{S}$ oligomers were prepared in the appropriate buffer by buffer exchange from a stock solution, using a Zeba Spin desalting column (Pierce).

\section{References}

(1) Lashuel, H. A., Hartley, D., Petre, B. M., Walz, T., and Lansbury, P. T., Jr. (2002) Neurodegenerative disease: amyloid pores from pathogenic mutations. Nature 418, 291.

(2) Furukawa, K., Matsuzaki-Kobayashi, M., Hasegawa, T., Kikuchi, A., Sugeno, N., Itoyama, Y., Wang, Y., Yao, P. J., Bushlin, I., and Takeda, A. (2006) Plasma membrane ion permeability induced by mutant alpha-synuclein contributes to the degeneration of neural cells. J. Neurochem. 97, 1071-1077.

(3) Mattson, M. P. (2007) Calcium and neurodegeneration. Aging Cell 6, 337-350.

(4) Volles, M. J., Lee, S. J., Rochet, J. C., Shtilerman, M. D., Ding, T. T., Kessler, J. C., and Lansbury, P. T., Jr. (2001) Vesicle permeabilization by protofibrillar alpha-synuclein: implications for the pathogenesis and treatment of Parkinson's disease. Biochemistry 40, 7812-7819.

(5) Kayed, R., Sokolov, Y., Edmonds, B., McIntire, T. M., Milton, S. C., Hall, J. E., and Glabe, C. G. (2004) Permeabilization of lipid bilayers is a common conformation-dependent activity of soluble amyloid oligomers in protein misfolding diseases. J. Biol. Chem. 279, 46363-46366.

(6) Quist, A., Doudevski, I., Lin, H., Azimova, R., Ng, D., Frangione, B., Kagan, B., Ghiso, J., and Lal, R. (2005) Amyloid ion channels: a common structural link for protein-misfolding disease. Proc. Natl. Acad. Sci. U. S. A. 102, 10427-10432. 
(7) Kostka, M., Högen, T., Danzer, K. M., Levin, J., Habeck, M., Wirth, A., Wagner, R., Glabe, C. G., Finger, S., Heinzelmann, U., Garidel, P., Duan, W., Ross, C. A., Kretzschmar, H., and Giese, A. (2008) Single particle characterization of iron-induced pore-forming alpha-synuclein oligomers. J. Biol. Chem. 283, 10992-11003.

(8) Volles, M. J., and Lansbury, P. T., Jr. (2002) Vesicle permeabilization by protofibrillar alpha-synuclein is sensitive to Parkinson's disease-linked mutations and occurs by a pore-like mechanism. Biochemistry 41, 4595-4602.

(9) Lashuel, H. A., Petre, B. M., Wall, J., Simon, M., Nowak, R. J., Walz, T., and Lansbury, P. T., Jr. (2002) Alpha-synuclein, especially the Parkinson's disease-associated mutants, forms porelike annular and tubular protofibrils. J. Mol. Biol. 322, 10891102.

(10) Ding, T. T., Lee, S. J., Rochet, J. C., and Lansbury, P. T., Jr. (2002) Annular alpha-synuclein protofibrils are produced when spherical protofibrils are incubated in solution or bound to brainderived membranes. Biochemistry 41, 10209-10217.

(11) Zhu, M., Li, J., and Fink, A. L. (2003) The association of alphasynuclein with membranes affects bilayer structure, stability, and fibril formation. J. Biol. Chem. 278, 40186-40197.

(12) van den Brink-van der Laan, E., Killian, J. A., and de Kruijff, B. (2004) Nonbilayer lipids affect peripheral and integral membrane proteins via changes in the lateral pressure profile. Biochim. Biophys. Acta 1666, 275-288.

(13) Marsh, D. (2007) Lateral pressure profile, spontaneous curvature frustration, and the incorporation and conformation of proteins in membranes. Biophys. J. 93, 3884-3899.

(14) Zimmerberg, J., and Kozlov, M. M. (2006) How proteins produce cellular membrane curvature. Nat. Rev. Mol. Cell Biol. 7, 9-19.

(15) Kooijman, E. E., Chupin, V., Fuller, N. L., Kozlov, M. M., de Kruijff, B., Burger, K. N., and Rand, P. R. (2005) Spontaneous curvature of phosphatidic acid and lysophosphatidic acid. Biochemistry 44, 2097-2102.

(16) Ipsen, J. H., Mouritsen, O. G., and Bloom, M. (1990) Relationships between lipid membrane area, hydrophobic thickness, and acyl-chain orientational order. The effects of cholesterol. Biophys. J. 57, 405-412. 
Lipid bilayer disruption by oligomeric $\alpha$-synuclein

(17) Nuscher, B., Kamp, F., Mehnert, T., Odoy, S., Haass, C., Kahle, P. J., and Beyer, K. (2004) Alpha-synuclein has a high affinity for packing defects in a bilayer membrane: a thermodynamics study. J. Biol. Chem. 279, 21966-21975.

(18) Lowe, R., Pountney, D. L., Jensen, P. H., Gai, W. P., and Voelcker, N. H. (2004) Calcium(II) selectively induces alpha-synuclein annular oligomers via interaction with the C-terminal domain. Protein Sci. 13, 3245-3252.

(19) Tamamizu-Kato, S., Kosaraju, M. G., Kato, H., Raussens, V., Ruysschaert, J. M., and Narayanaswami, V. (2006) Calciumtriggered membrane interaction of the alpha-synuclein acidic tail. Biochemistry 45, 10947-10956.

(20) Tsigelny, I. F., Bar-On, P., Sharikov, Y., Crews, L., Hashimoto, M., Miller, M. A., Keller, S. H., Platoshyn, O., Yuan, J. X., and Masliah, E. (2007) Dynamics of alpha-synuclein aggregation and inhibition of pore-like oligomer development by beta-synuclein. FEBS J. 274, 1862-1877.

(21) Lee, H. J., Choi, C., and Lee, S. J. (2002) Membrane-bound alphasynuclein has a high aggregation propensity and the ability to seed the aggregation of the cytosolic form. J. Biol. Chem. 277, 671678.

(22) Zakharov, S. D., Hulleman, J. D., Dutseva, E. A., Antonenko, Y. N., Rochet, J. C., and Cramer, W. A. (2007) Helical alphaSynuclein Forms Highly Conductive Ion Channels. Biochemistry 46, 14369-14379.

(23) Choi, W., Zibaee, S., Jakes, R., Serpell, L. C., Davletov, B., Crowther, R. A., and Goedert, M. (2004) Mutation E46K increases phospholipid binding and assembly into filaments of human alpha-synuclein. FEBS Lett. 576, 363-368.

(24) Jensen, P. H., Nielsen, M. S., Jakes, R., Dotti, C. G., and Goedert, M. (1998) Binding of alpha-synuclein to brain vesicles is abolished by familial Parkinson's disease mutation. J. Biol. Chem. 273, 26292-26294.

(25) Davidson, W. S., Jonas, A., Clayton, D. F., and George, J. M. (1998) Stabilization of alpha-synuclein secondary structure upon binding to synthetic membranes. J. Biol. Chem. 273, 9443-9449.

(26) Jo, E., McLaurin, J., Yip, C. M., St George-Hyslop, P., and Fraser, P. E. (2000) alpha-Synuclein membrane interactions and lipid specificity. J. Biol. Chem. 275, 34328-34334. 
(27) Eliezer, D., Kutluay, E., Bussell, R., Jr., and Browne, G. (2001) Conformational properties of alpha-synuclein in its free and lipidassociated states. J. Mol. Biol. 307, 1061-1073.

(28) Allende, D., Simon, S. A., and McIntosh, T. J. (2005) Melittininduced bilayer leakage depends on lipid material properties: evidence for toroidal pores. Biophys. J. 88, 1828-1837.

(29) Sokolov, Y., Kozak, J. A., Kayed, R., Chanturiya, A., Glabe, C., and Hall, J. E. (2006) Soluble amyloid oligomers increase bilayer conductance by altering dielectric structure. J. Gen. Physiol. 128, 637-647.

(30) Kayed, R., Head, E., Thompson, J. L., McIntire, T. M., Milton, S. C., Cotman, C. W., and Glabe, C. G. (2003) Common structure of soluble amyloid oligomers implies common mechanism of pathogenesis. Science 300, 486-489.

(31) Danzer, K. M., Haasen, D., Karow, A. R., Moussaud, S., Habeck, M., Giese, A., Kretzschmar, H., Hengerer, B., and Kostka, M. (2007) Different species of alpha-synuclein oligomers induce calcium influx and seeding. J. Neurosci. 27, 9220-9232.

(32) van Meer, G., Voelker, D. R., and Feigenson, G. W. (2008) Membrane lipids: where they are and how they behave. Nat. Rev. Mol. Cell Biol. 9, 112-124.

(33) Lee, S. J. (2008) Origins and effects of extracellular alphasynuclein: implications in Parkinson's disease. J. Mol. Neurosci. $34,17-22$.

(34) Cookson, M. R., and van der Brug, M. (2008) Cell systems and the toxic mechanism(s) of alpha-synuclein. Exp. Neurol. 209, $5-11$.

(35) Li, W. W., Yang, R., Guo, J. C., Ren, H. M., Zha, X. L., Cheng, J. S., and Cai, D. F. (2007) Localization of alpha-synuclein to mitochondria within midbrain of mice. Neuroreport 18, 15431546 .

(36) Shavali, S., Brown-Borg, H. M., Ebadi, M., and Porter, J. (2008) Mitochondrial localization of alpha-synuclein protein in alphasynuclein overexpressing cells. Neurosci. Lett. 439, 125-128.

(37) Cole, N. B., Dieuliis, D., Leo, P., Mitchell, D. C., and Nussbaum, R. L. (2008) Mitochondrial translocation of alpha-synuclein is promoted by intracellular acidification. Exp. Cell. Res. 314, 20762089.

(38) Ramakrishnan, M., Jensen, P. H., and Marsh, D. (2003) Alphasynuclein association with phosphatidylglycerol probed by lipid spin labels. Biochemistry 42, 12919-12926. 
(39) Stichel, C. C., Zhu, X. R., Bader, V., Linnartz, B., Schmidt, S., and Lübbert, H. (2007) Mono- and double-mutant mouse models of Parkinson's disease display severe mitochondrial damage. Hum. Mol. Genet. 16, 2377-2393.

(40) Devi, L., Raghavendran, V., Prabhu, B. M., Avadhani, N. G., and Anandatheerthavarada, H. K. (2008) Mitochondrial import and accumulation of alpha-synuclein impair complex I in human dopaminergic neuronal cultures and Parkinson disease brain. J. Biol. Chem. 283, 9089-9100.

(41) Parihar, M. S., Parihar, A., Fujita, M., Hashimoto, M., and Ghafourifar, P. (2008) Mitochondrial association of alphasynuclein causes oxidative stress. Cell Mol. Life Sci. 65, 12721284.

(42) Chen, P. S., Toribara, T. Y., and Warner, H. (1956) Microdetermination of Phosphorus. Anal. Chem. 28, 1756-1758. 



\section{Chapter 4 Membrane binding of oligomeric $\alpha$-synuclein}

Membrane disruption by oligomeric $\alpha$-synuclein is considered a likely mechanism of cytotoxicity in Parkinson's disease. However, the mechanism of oligomer membrane binding and the relation between binding and membrane disruption is not known. We have measured oligomer-lipid binding by fluorescence microscopy and have measured membrane disruption using a dye release assay. Oligomer-lipid binding can be visualized by confocal microscopy using fluorescently labeled oligomers and giant unilamellar vesicles. The data reveal that oligomeric $\alpha$-synuclein selectively binds to vesicles containing anionic lipids. When oligomers were added to vesicles containing coexisting ordered and disordered domains, oligomeric $\alpha$-synuclein preferentially accumulated into the liquid disordered domains. Furthermore, we show that binding of oligomers to the membrane and disruption of the membrane require different lipid properties. Thus membrane-bound oligomeric $\alpha$-synuclein does not cause bilayer disruption under all conditions. 


\section{Introduction}

The lipid interaction of $\alpha \mathrm{S}$ has been the subject of intensive research over the past decade for a number of reasons. The interaction of monomeric $\alpha \mathrm{S}$ with cellular membranes has been suggested to play a possible role in its biological function (1). In addition, the membrane environment could play an important role in modulating $\alpha \mathrm{S}$ aggregation (2). In recent years, oligomeric intermediates in the aggregation of $\alpha \mathrm{S}$ are considered as a likely toxic species $(3,4)$. Membrane disruption and subsequent calcium leakage have been suggested as a possible mechanism of oligomer toxicity (5). The interaction of monomeric $\alpha \mathrm{S}$ with phospholipids is well characterized. Monomeric $\alpha \mathrm{S}$ binds to negatively charged phospholipid vesicles in the liquid disordered (Ld) phase (6-8). Binding of $\alpha \mathrm{S}$ monomers to membranes is mediated by the N-terminus, which folds into an $\alpha$-helical conformation (9).

Although oligomer-membrane disruption is a possible toxic mechanism in $\mathrm{PD}$, very little is known about the lipid binding of $\alpha \mathrm{S}$ oligomers. In vitro oligomeric $\alpha \mathrm{S}$ can permeabilize lipid vesicles, possibly through a pore-like mechanism (10-12). As was shown in the previous chapter, vesicle permeabilization is primarily dependent on the bilayer charge (11-14). This could be related to the binding specificity of oligomeric $\alpha \mathrm{S}$. Alternatively, oligomeric $\alpha \mathrm{S}$ could also bind to less negatively charged membranes, but only disrupt the membrane under specific conditions.

Using confocal fluorescence microscopy we have investigated the lipid binding properties of oligomeric $\alpha \mathrm{S}$. In addition, quantitative results from binding experiments and a dye efflux assay were used to study the relation between binding and disruption of the membrane. Confocal microscopy images on giant unilamellar vesicles (GUVs) and fluorescently labeled oligomeric $\alpha \mathrm{S}$ indicate that oligomers preferentially bind to GUVs composed of anionic phospholipids in the Ld phase. Furthermore, we observe that the binding of oligomers to the negatively charged vesicle bilayer does not necessarily result in membrane permeabilization. The lipid headgroup composition determines whether binding can result in disruption of the bilayer. Thus, lipid binding and membrane permeabilization by oligomeric $\alpha \mathrm{S}$ are distinct processes.

\section{Results}

\section{Lipid binding headgroup specificity}

To obtain fluorescently labeled oligomers, $\alpha$ S-A140C-AL488 was mixed with $\alpha \mathrm{S}$-wt in a 1:7 ratio. Labeled $\alpha \mathrm{S}$ efficiently incorporated into the oligomeric species and the labeling ratio was spectroscopically determined to be 10-15 $\%$. After purification no monomeric $\alpha \mathrm{S}$ could be detected in the oligomer 


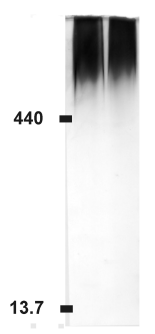

A

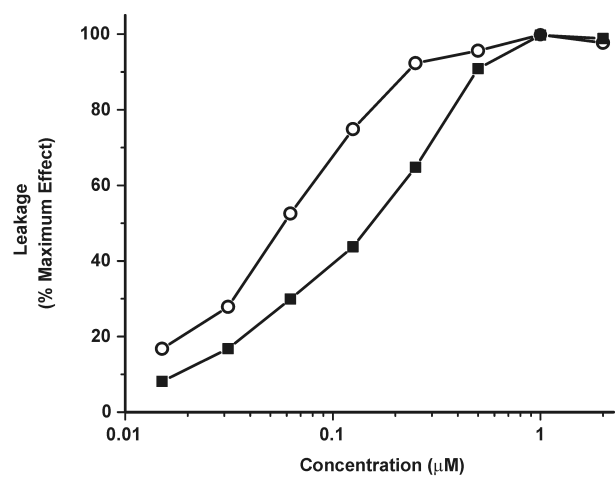

B

Figure 1. Oligomer characterization (A) Native gradient gel of $\alpha \mathrm{S}$-wt oligomers (left lane) and $15 \%$ labeled $\alpha \mathrm{S}$ oligomers (right lane). Gels were cast with a polyacrylamide gradient from 3.5 to $15 \%$. Silver staining was applied for sensitive detection. The markers ferritin $(440 \mathrm{kDa})$ and Ribonuclease A $(13.7 \mathrm{kDa})$ were also applied on the gel. (B) Calcein leakage from POPG LUVs, induced by $\alpha \mathrm{S}-\mathrm{wt}$ oligomers (squares) and $\alpha$ S-wt/A140C-AL488 oligomers (open circles). Leakage is expressed as a percentage of the maximum effect induced by the protein.

solution by native gradient PAGE (Figure 1A), which ensured that the observed binding signal is not caused by monomeric $\alpha \mathrm{S}$. The labeled oligomers migrated at a similar molecular weight as $\alpha \mathrm{S}$-wt oligomers and showed a comparable tendency to disrupt POPG vesicles as the unlabeled species (Figure 1B). It can therefore be concluded that the introduction of $\alpha \mathrm{S}-\mathrm{A} 140 \mathrm{C}-\mathrm{AL} 488$ does not affect the oligomer properties.

The binding of oligomers to GUVs was studied using confocal fluorescence microscopy. First GUVs from different anionic lipids with $20 \%$ cholesterol were prepared. Labeled $\alpha \mathrm{S}$ oligomers clearly showed binding to these negatively charged GUVs (Figure 2 A-C). GUV morphology was not affected by binding of oligomeric $\alpha \mathrm{S}$ and the GUVs remained stable during the time of the experiment. Binding of $\alpha \mathrm{S}$ oligomers to neutrally charged POPC GUVs could not be detected (Figure 2D). Increasing the protein to lipid ratio 12 times to $\sim 1: 5$ did not change this observation. To further investigate the specificity for negatively charged headgroups, 1:1 mixtures of POPC and anionic phospholipids were used (Figure 3). The $\alpha \mathrm{S}$ oligomers showed binding to POPC:DOPA and POPC:POPG GUVs but much lower binding was detected to POPC:POPS. The addition of zwitterionic lipids thus does not fully inhibit the membrane binding of oligomeric $\alpha \mathrm{S}$ but may influence the membrane affinity of the oligomers. 


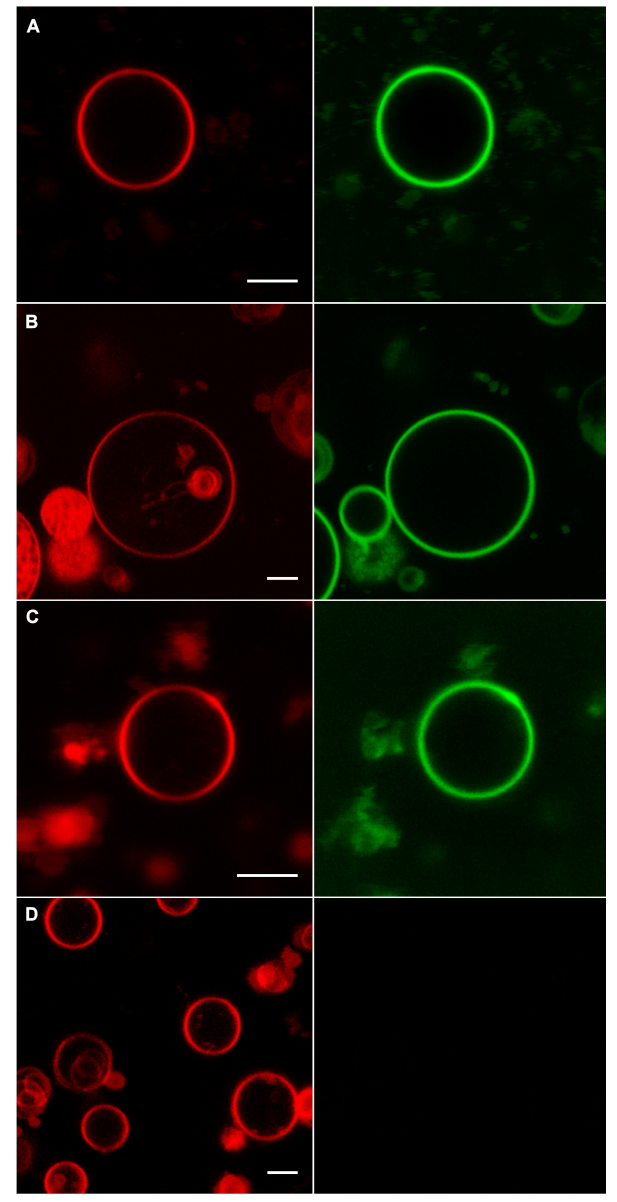

Figure 2. Headgroup specificity Confocal Microscopy images of DOPE-Rhod labeled GUVs (left panels) and $\alpha$ S-A140C-AL488 oligomers (right panels). The scale bars indicate $5 \mu \mathrm{m}$. The lipid compositions of the GUVs were, (A): POPG:Chol 5:1, (B): DOPA:Chol 5:1, (C): POPS:Chol 5:1, (D): POPC. 


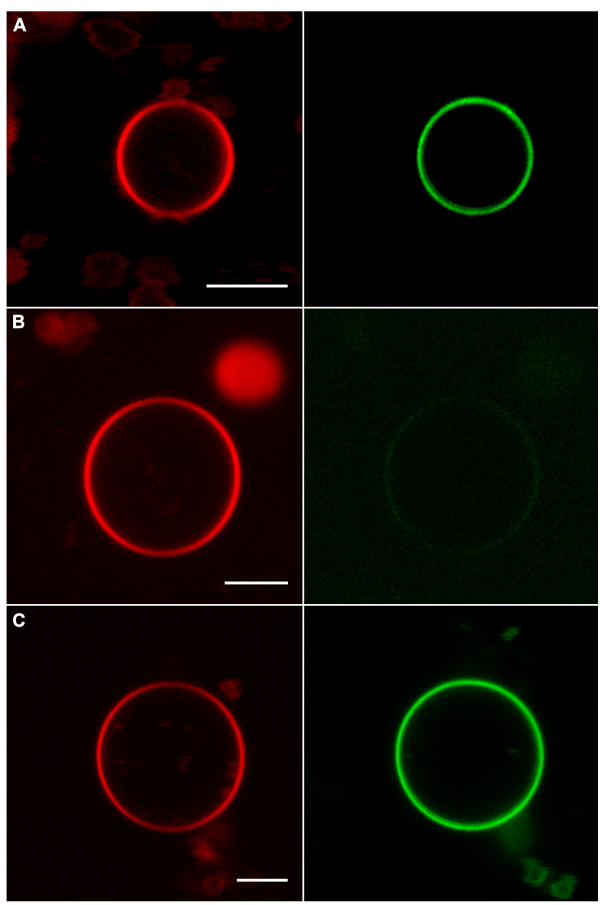

Figure 3. Mixed bilayers Confocal microscopy images of DOPE-Rhod labeled GUVs from 1:1 mixture of anionic lipids with POPC (left panels) and $\alpha$ S-A140CAL488 oligomers (right panels). The scale bars indicate $5 \mu \mathrm{m}$. The lipid compositions of the GUVs were, (A): POPC:POPG, (B) POPC: POPS, (C): POPC:DOPA.

\section{Oligomer membrane permeabilization}

The lipid disruption properties of oligomeric $\alpha \mathrm{S}$ are critically dependent on the lipid headgroup composition $(11,13)$. As was shown in the previous chapter, negatively charged vesicles are permeabilized upon addition of oligomeric $\alpha \mathrm{S}$, which is generally inhibited upon the addition of zwitterionic lipids to the membrane composition. To investigate if this inhibitory effect originates from a different binding affinity or from a different mode of binding, a dye release assay together with a quantitive binding experiment was performed. The results shown in Figure 4 indicate that POPC:POPG LUVs are not permeabilized, whereas POPC:DOPA LUVs showed a concentration dependent calcein release upon addition of oligomeric $\alpha \mathrm{S}$. Next, labeled $\alpha \mathrm{S}$ oligomers were added to POPC:POPG and POPC:DOPA GUVs at different concentrations and bind- 


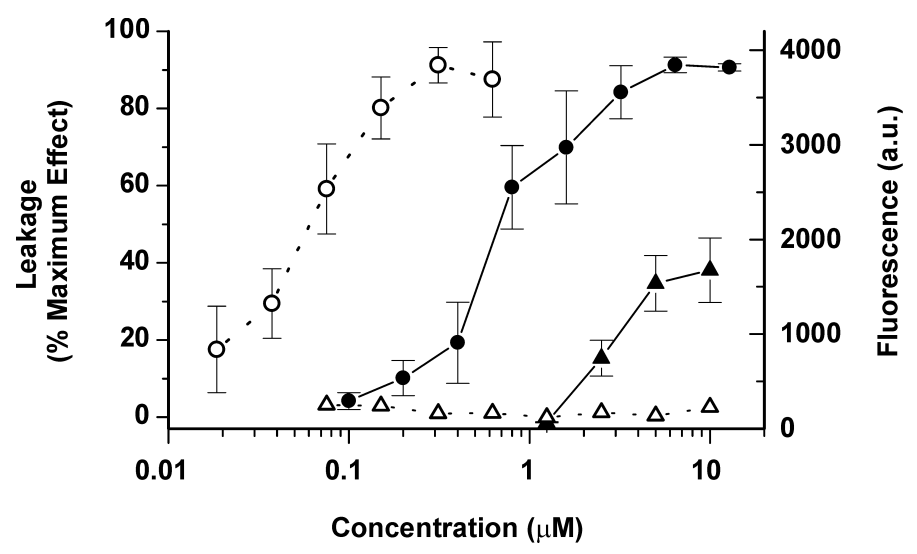

Figure 4. Binding and permeabilization Calcein release from LUVs (open symbol, dotted lines) and binding to GUVs (closed symbols, solid lines) from POPC:DOPA (circles) and POPC:POPG vesicles (triangles), all at a lipid concentration of $40 \mu \mathrm{M}$ and at different $\alpha \mathrm{S}$-A140C-AL488 concentrations (oligomer concentration is expressed in equivalent monomer concentration). The error bars denote the standard deviation $(n=2)$.

ing was quantified from the fluorescence intensity on the membrane in the confocal images. These results indicate that $\alpha \mathrm{S}$ oligomers bind POPC:DOPA with higher affinity than the POPC:POPG GUVs, but the difference in binding affinity is much smaller than the difference in permeabilization properties. The degree of vesicle permeabilization is thus not directly related to the amount of protein on the membrane, but may depend on the bilayer structure and the actual mode of binding.

\section{Lipid binding phase specificity}

Lipid packing can be a critical parameter in determining protein lipid interactions. We have shown in the previous chapter that vesicle disruption by oligomeric $\alpha \mathrm{S}$ is sensitive to lipid packing (13). Denser packed membranes were found to be less sensitive to disruption. Lipids in the liquid ordered (Lo) phase are much more densely packed than lipids in the Ld phase. Thus, the effect of lipid phase on $\alpha \mathrm{S}$ oligomer lipid binding was examined. A binding specificity to Ld or Lo phases can be visualized by examining binding to GUVs containing coexisting domains. This can be achieved since DOPE-Rhod preferentially accumulates in Ld domains (15). 18:2 PG:DPPG:Chol GUVs were prepared and formation of segregated Ld and Lo domains was observed (Figure $5 \mathrm{~A}$ ). The fluorescently labeled oligomers preferentially accumulated in the 


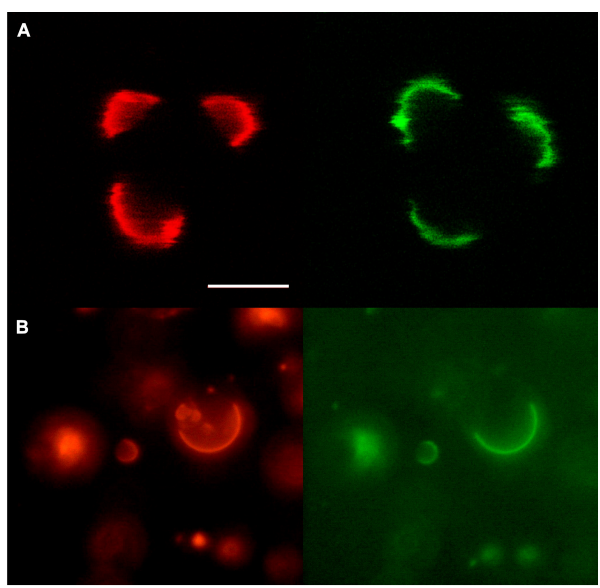

Figure 5. Phase specificity Confocal microscopy image (A) and wide field fluorescence microscopy image (B) of DOPE-Rhod labeled 18:2 PG:DPPG:Chol 1:1:1 GUVs (left panels) and $\alpha$ S-A140C-AL488 oligomers (right panels). The scale bar indicates $5 \mu \mathrm{m}$.

DOPE-Rhod rich domains. This specificity for Ld domains was also observed using widefield fluorescence microscopy (Figure 5B). In addition, for GUVs of other compositions containing only lipids in the Lo phase, no oligomer binding could be detected (Figure 6). Oligomeric $\alpha \mathrm{S}$ thus preferentially binds to lipids in the Ld phase.

\section{Discussion}

$\alpha S$ oligomers only bind to anionic lipids

$\alpha \mathrm{S}$ oligomers preferentially bind to GUVs consisting of anionic lipids and no binding was detected to POPC GUVs. Oligomer binding was observed in mixtures of anionic lipids and POPC such as POPC:POPG and POPC:DOPA GUVs, while little binding to POPC:POPS was observed. Differences in lipid headgroup structure might be responsible for the apparently lower affinity of $\alpha \mathrm{S}$ oligomers for POPS in mixed GUVs. Another likely explanation is that the difference is related to the fact that, at room temperature, POPS is close to its phase transition temperature. We have previously observed calcein efflux from DOPS LUVs, but POPS LUVs proved not to be sensitive to disruption by oligomeric $\alpha \mathrm{S}$ (See chapter 3). Thus, the lower binding to POPS might be related to packing parameters and not chemical headgroup structure. 


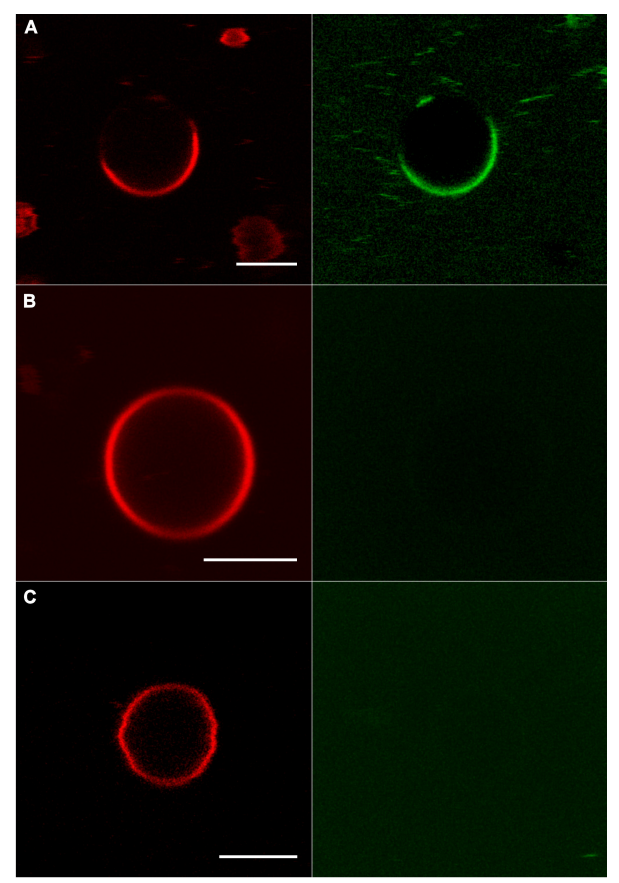

Figure 6. Phase specificity Confocal microscopy images of DOPE-Rhod labeled GUVs (left panels) and $\alpha$ S-wt/140C-AL488 oligomers (right panels). Scale bars indicate $5 \mu \mathrm{m}$. (A) 18:2 PG:DPPG:Chol 1:1:1 GUVs (B) No domain formation was observed in the POPG:DPPG:chol GUVs. Although the phase state for this composition is not exactly known, it is likely more densely packed than DOPG:Chol 5:1 GUVs. (C) DPPG has a phase transition temperature of $\sim 55{ }^{\circ} \mathrm{C}$. GUVs prepared from this lipid are in the Lo phase at room temperature. GUVs from DPPG:Chol 4:1 were difficult to obtain; the few detected GUVs did not bind $\alpha \mathrm{S}$ oligomers. 
Membrane binding of oligomeric $\alpha$-synuclein

The binding preference for anionic lipids agrees with an earlier report, indicating binding to PG and PC:PA but not to PC (12). However, the authors did not check binding to other lipids and lipid mixtures. Others have reported that during aggregation of $\alpha \mathrm{S}$, intermediates with distinct lipid binding properties occur (16). Possibly, different intermediates in the aggregation of $\alpha \mathrm{S}$ could possess different lipid binding properties. Detailed binding studies require stable purified samples. For earlier intermediates in the aggregation this might be technically challenging.

\section{Binding does not always lead to permeabilization}

In spite of the observation that $\alpha \mathrm{S}$ oligomers bind POPC:POPG GUVs, the dye release experiments show that the POPC:POPG LUVs are not permeabilized. The quantitative imaging experiment revealed a smaller binding affinity for POPC:POPG compared to POPC:DOPA LUVs. However, this difference is too small to explain why POPC:POPG LUVs are not affected even at high oligomer concentrations. Thus, binding of $\alpha \mathrm{S}$ oligomers to the membrane does not necessarily lead to membrane permeabilization. We postulate that membrane disruption is a two step process in which the initial binding and the subsequent disruption require different lipid properties. The mechanism of permeabilization is currently still debated $(10,11,17)$. When comparing the concentration dependence of leakage with the binding curves it appears that the membrane does not need to be saturated with oligomers before leakage occurs, which is consistent with a pore model. However, one must be cautious in comparing the GUV and LUV systems. Especially GUV containing solutions are extremely heterogeneous and therefore a quantitative comparison between result from LUVs and GUVs may not be valid under all conditions.

\section{$\alpha S$ Oligomers only bind to Ld domains}

Lipid packing is an important parameter in determining protein lipid interactions. We have previously shown that bilayer disruption is sensitive to the accessibility of the hydrophobic bilayer core (13). The results presented here are consistent with these data, and show that $\alpha \mathrm{S}$ oligomers preferentially bind to lipids in the Ld phase and do not show affinity for the densely packed Lo bilayers. Possibly, the higher surface charge density in the Lo phase makes it energetically unfavorable for the negatively charged $\alpha \mathrm{S}$ to adsorb on the membrane. Alternatively, steric hindrance, inaccessibility of the membrane hydrophobic core, or membrane mechanical properties offer possible explanations for why oligomeric $\alpha \mathrm{S}$ does not bind to the Lo phase. Interestingly, monomeric $\alpha \mathrm{S}$ has a similar binding specificity for anionic lipids in the Ld phase $(6,7)$. Lipid binding of monomeric $\alpha \mathrm{S}$ occurs at residues 1-100 (9). Although the protein has a net negative charge, the $\mathrm{N}$-terminal residues contain many hy- 
drophobic and positively charged amino acids, explaining the specificity for anionic lipids. The similar specificity for oligomeric $\alpha \mathrm{S}$ could suggest that in the oligomers, the N-terminal is available for lipid binding. We have not found evidence for a structural reorganization of $\alpha \mathrm{S}$ oligomers upon lipid interaction by circular dichroism spectroscopy, consistent with earlier work (12). Folding of the N-terminus into a helical conformation upon lipid binding as occurs with monomeric $\alpha \mathrm{S}$ is therefore unlikely for oligomeric $\alpha \mathrm{S}$.

Summarizing, we have systematically studied the lipid binding properties of oligomeric $\alpha \mathrm{S}$ and its relation to bilayer disruption. The data show that oligomeric $\alpha \mathrm{S}$ preferentially binds to anionic lipids in the Ld phase. Furthermore, binding does not lead to membrane disruption for all lipid compositions, which suggests that binding and disruption are distinct processes.

\section{Materials and Methods}

Oligomer preparation. Preparation of $\alpha \mathrm{S}$ and fluorescently labeled oligomers was performed as described in Chapter 2. Again $\alpha$ S-wt/A140C-AL488 oligomers were prepared at $\sim 20 \mu \mathrm{M}$ (equivalent monomer concentration) and 10-15 $\%$ labeling.

Vesicle preparation. 1,2-dipalmitoyl phosphatidylglycerol (DPPG), 1,2-dilinoleoyl phosphatidylglycerol (18:2-PG), 1,2-dioleoyl phosphatidylethanolamine(Lissamine Rhodamine B) (DOPE-Rhod), POPC, POPG, POPS, DOPA, and cholesterol were obtained from Avanti Polar lipids. Vesicles of various compositions were prepared by mixing the lipids from stock solutions in chloroform. $0.05 \%$ DOPE-Rhod was added to all compositions. GUVs containing anionic lipids were created by gentle hydration. Approximately $0.5 \mathrm{mg}$ of lipid was deposited in a glass vial and dried using nitrogen gas. Residual chloroform was removed by drying under vacuum for 4 hours. The lipid film was hydrated for 6 hours at room temperature in $500 \mu \mathrm{l}$ of sucrose solution of equal osmolarity to $10 \mathrm{mM}$ HEPES pH 7.4, $150 \mathrm{mM} \mathrm{NaCl}$. GUVs containing saturated lipids were hydrated at $50^{\circ} \mathrm{C}$. Electroswelling (18) was used to prepare POPC GUVs. A dried lipid film was prepared on the conducting side of an ITO coated slide and joined with a second ITO slide separated by a spacer and hydrated overnight at room temperature in $400 \mu \mathrm{l}$ of sucrose solution in the presence of an $\mathrm{AC}$ electric field $(1 \mathrm{~V} / \mathrm{mm}, 10 \mathrm{~Hz})$.

Confocal microscopy. Confocal microscopy was performed on a Zeiss LSM 510 confocal microscope. DOPE-Rhod was excited at $543 \mathrm{~nm}$ using a green HeNe laser and $\alpha$ S-AL488 was excited using the $488 \mathrm{~nm}$ Argon laser line. Fluorescence was detected using multitrack imaging to minimize crosstalk. GUVs were diluted to approximately $60 \mu \mathrm{M}$ in $10 \mathrm{mM}$ Hepes, pH 7.4, $150 \mathrm{mM} \mathrm{NaCl}$ and mixed with fluorescently labeled oligomeric $\alpha \mathrm{S}$ at a $1 \mu \mathrm{M}$ concentration 
Membrane binding of oligomeric $\alpha$-synuclein

(equivalent monomer concentration). In the quantitive binding experiments images were acquired at exactly the same instrument settings. The amount of binding was quantified from the GUV images as the maximum value of the radial profile. For each data point images of at least $15 \mathrm{GUVs}$ were acquired. The experiment was performed twice.

Dye release assay The dye release assay was performed as described in Chapter 3.

\section{References}

(1) Beyer, K. (2007) Mechanistic aspects of Parkinson's disease: alpha-synuclein and the biomembrane. Cell Biochem. Biophys. 47, 285-299.

(2) Lee, H. J., Choi, C., and Lee, S. J. (2002) Membrane-bound alphasynuclein has a high aggregation propensity and the ability to seed the aggregation of the cytosolic form. J. Biol. Chem. 277, 671678 .

(3) Kayed, R., Head, E., Thompson, J. L., McIntire, T. M., Milton, S. C., Cotman, C. W., and Glabe, C. G. (2003) Common structure of soluble amyloid oligomers implies common mechanism of pathogenesis. Science 300, 486-489.

(4) Lashuel, H. A., and Lansbury, P. T., Jr. (2006) Are amyloid diseases caused by protein aggregates that mimic bacterial poreforming toxins? Q. Rev. Biophys. 39, 167-201.

(5) Lashuel, H. A., Hartley, D., Petre, B. M., Walz, T., and Lansbury, P. T., Jr. (2002) Neurodegenerative disease: amyloid pores from pathogenic mutations. Nature 418, 291.

(6) Davidson, W. S., Jonas, A., Clayton, D. F., and George, J. M. (1998) Stabilization of alpha-synuclein secondary structure upon binding to synthetic membranes. J. Biol. Chem. 273, 9443-9449.

(7) Stöckl, M., Fischer, P., Wanker, E., and Herrmann, A. (2008) Alpha-synuclein selectively binds to anionic phospholipids embedded in liquid-disordered domains. J. Mol. Biol. 375, 1394-1404.

(8) Rhoades, E., Ramlall, T. F., Webb, W. W., and Eliezer, D. (2006) Quantification of alpha-synuclein binding to lipid vesicles using fluorescence correlation spectroscopy. Biophys. J. 90, 4692-4700.

(9) Eliezer, D., Kutluay, E., Bussell, R., Jr., and Browne, G. (2001) Conformational properties of alpha-synuclein in its free and lipidassociated states. J. Mol. Biol. 307, 1061-1073. 
(10) Quist, A., Doudevski, I., Lin, H., Azimova, R., Ng, D., Frangione, B., Kagan, B., Ghiso, J., and Lal, R. (2005) Amyloid ion channels: a common structural link for protein-misfolding disease. Proc. Natl. Acad. Sci. U. S. A. 102, 10427-10432.

(11) Volles, M. J., and Lansbury, P. T., Jr. (2002) Vesicle permeabilization by protofibrillar alpha-synuclein is sensitive to Parkinson's disease-linked mutations and occurs by a pore-like mechanism. Biochemistry 41, 4595-4602.

(12) Volles, M. J., Lee, S. J., Rochet, J. C., Shtilerman, M. D., Ding, T. T., Kessler, J. C., and Lansbury, P. T., Jr. (2001) Vesicle permeabilization by protofibrillar alpha-synuclein: implications for the pathogenesis and treatment of Parkinson's disease. Biochemistry 40, 7812-7819.

(13) van Rooijen, B. D., Claessens, M. M., and Subramaniam, V. (2009) Lipid bilayer disruption by oligomeric alpha-synuclein depends on bilayer charge and accessibility of the hydrophobic core. Biochim. Biophys. Acta Biomembr. 1788, 1271-1278.

(14) Zhu, M., Li, J., and Fink, A. L. (2003) The association of alphasynuclein with membranes affects bilayer structure, stability, and fibril formation. J. Biol. Chem. 278, 40186-40197.

(15) Baumgart, T., Hess, S. T., and Webb, W. W. (2003) Imaging coexisting fluid domains in biomembrane models coupling curvature and line tension. Nature 425, 821-824.

(16) Smith, D. P., Tew, D. J., Hill, A. F., Bottomley, S. P., Masters, C. L., Barnham, K. J., and Cappai, R. (2008) Formation of a high affinity lipid-binding intermediate during the early aggregation phase of alpha-synuclein. Biochemistry 47, 1425-1434.

(17) Kayed, R., Sokolov, Y., Edmonds, B., McIntire, T. M., Milton, S. C., Hall, J. E., and Glabe, C. G. (2004) Permeabilization of lipid bilayers is a common conformation-dependent activity of soluble amyloid oligomers in protein misfolding diseases. J. Biol. Chem. 279, 46363-46366.

(18) Angelova, N., Soleau, S., Meleard, P., Faucon, J.F. and Bothorel, P. (1992) Preperation of giant vesicles by external AC electric fields. Kinetics and applications. Prog. Colloid Polym. Sci. 89, 127-131. 


\section{Chapter 5 Quantifying oligomer-lipid binding using fluorescence correlation spectroscopy}

Membrane permeabilization by oligomeric aggregates of the neuronal protein $\alpha$-synuclein is considered a possible cytotoxic mechanism in Parkinson's disease. To be able to evaluate the relevance of membrane permeabilization in the disease process, it is important to understand the lipid binding properties of oligomeric $\alpha$-synuclein and the structural elements supporting lipid-binding. We have quantified the binding of oligomeric $\alpha$-synuclein to large unilamellar vesicles with fluorescence correlation spectroscopy. By changing the membrane composition and solution conditions we have studied the role of charge interactions in the binding process. Oligomeric $\alpha$-synuclein binds to lipid vesicles with a slightly lower affinity than monomeric protein, with a dissociation constant in the micromolar range. The binding of oligomers to the membrane is strongly dependent on membrane charge, as binding requires the presence of large amounts of negatively charged lipids in the membrane. The specific binding to anionic lipids is remarkable since $\alpha$-synuclein is highly negatively charged. Correspondingly, reduced Debye screening at low ionic strengths inhibits the binding of the oligomers to the vesicles. The binding specificity for anionic lipids at physiological salt concentrations implies a specific charge distribution on the oligomer. Positively charged patches on the oligomer surface or conformational flexibility of the negatively charged C-terminus most likely play a role in mediating the lipid binding of the negatively charged oligomeric $\alpha$-synuclein. 


\section{Introduction}

The neuronal protein $\alpha \mathrm{S}$ is a possible key player in the onset and progression of PD. Aggregated fibrillar $\alpha \mathrm{S}$ is the major component of Lewy bodies (1), the intracellular inclusion bodies that are the pathological hallmark of PD. Although the aggregation of $\alpha \mathrm{S}$ has been extensively studied, how and if $\alpha \mathrm{S}$ fibrils are related to neuronal toxicity is not established (2). In recent years attention has shifted to earlier oligomeric intermediates in the aggregation of $\alpha \mathrm{S}$ as the possible toxic species. Membrane permeabilization by these oligomeric species is a likely mechanism for toxicity (3). Although membrane interactions of oligomeric $\alpha \mathrm{S}$ have been suggested as a possible mechanism of toxicity, very little is known about the lipid binding properties of oligomeric $\alpha \mathrm{S}$ and how these are related to the structural properties of the oligomers.

In this chapter we have used FCS to quantify the lipid binding of oligomeric $\alpha \mathrm{S}$ to LUVs and have investigated in detail the role of charge interactions. Oligomeric $\alpha \mathrm{S}$ specifically binds vesicles composed of negatively charged lipids with a dissociation constant in the micromolar range. Since $\alpha \mathrm{S}$ is highly negatively charged at neutral $\mathrm{pH}$, binding to a negatively charged membrane suggests a distinct charge distribution on the oligomer surface. A charge separation of the protein in the oligomeric complex could maximize favorable charge interactions with the bilayer. The requirement of well-defined positively charged patches on the $\alpha \mathrm{S}$, suggests that the oligomers have defined structural features.

\section{Results and Discussion}

In order to measure the lipid binding of oligomeric $\alpha \mathrm{S}$ to LUVs with FCS, fluorescently labeled oligomeric $\alpha \mathrm{S}$ was prepared similar as described in Chapter 2 and 4. Approximately $10 \%$ of Alexa 488 (AL488) labeled single cysteine mutant $\alpha \mathrm{S}-140 \mathrm{C}-\mathrm{AL} 488$ was incorporated into oligomeric $\alpha \mathrm{S}$. The diffusion time through the confocal detection volume was estimated by fitting the correlation curves (Figure 1A) with a free diffusion model (Appendix A, Equation 2). Monomeric $\alpha \mathrm{S}$ had a diffusion time of approximately $260 \mu \mathrm{s}$. The diffusion time of the oligomeric species varied slightly from batch to batch (950-1000 $\mu \mathrm{s})$. The diffusion time of the LUVs was generally around 4.5 to $5.5 \mathrm{~ms}$. The difference in diffusion time between free and LUV-bound protein allows for fitting the correlation curves with a two-component diffusion model (Appendix A, equation 4). In this way the fraction of bound protein can be estimated.

First the binding of $\alpha \mathrm{S}-\mathrm{A} 140 \mathrm{C}-\mathrm{AL} 488$ and the AL488 labeled oligomers to negatively charged DOPS LUVs was studied. Both monomeric and oligomeric $\alpha \mathrm{S}$ showed binding to these vesicles (Figure 1B). The binding curves deviate only slightly from the Langmuir isotherm. Similar to Rhoades et al. (4), 

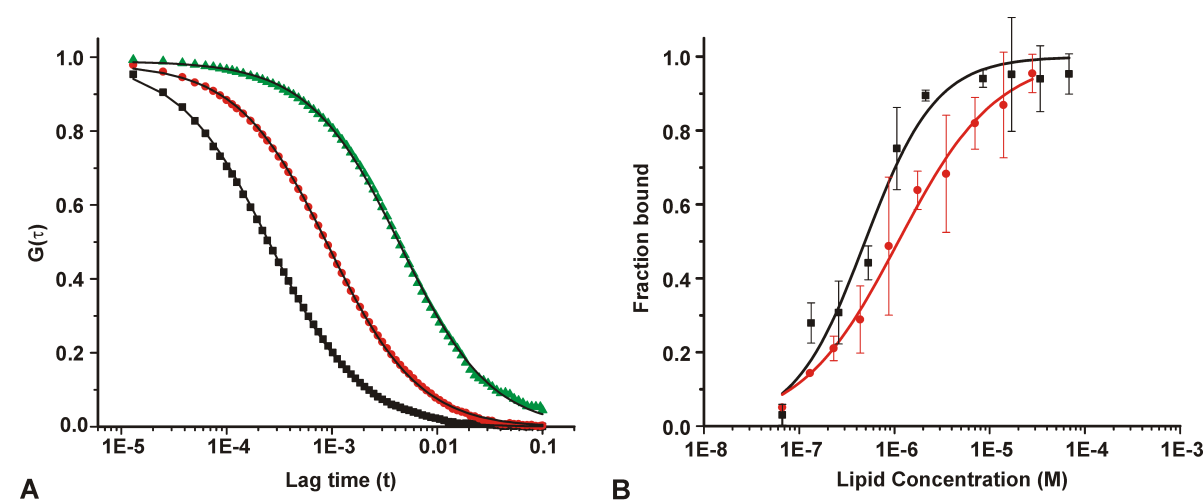

Figure 1. Oligomer-lipid binding (A) Normalized correlation curves of free monomeric $\alpha$ S-A140C-Al488 (black squares), AL488 labeled oligomeric $\alpha \mathrm{S}$ (red circles) and oligomeric $\alpha \mathrm{S}$ bound to DOPS LUVs (green triangles) fitted with a single component free diffusion model. (B) Binding curves of $50 \mathrm{nM} \alpha \mathrm{S}-\mathrm{A} 140 \mathrm{C}-\mathrm{AL} 488$ monomer (black squares) and $50 \mathrm{nM}$ oligomeric $\alpha \mathrm{S}$ (equivalent monomer concentration) to DOPS LUVs in the presence of $50 \mathrm{mM} \mathrm{NaCl}$. The solid lines represent a fit from the Hill model.

we have used the Hill equation to fit such binding curves. The dissociation constant $\left(K_{d}\right)$ for binding to the LUVs derived from the fit was $0.5 \mu \mathrm{M}$ for monomeric $\alpha \mathrm{S}$ and $1.1 \mu \mathrm{M}$ for oligomeric $\alpha \mathrm{S}$, with Hill coefficients of 1.2 and 0.8 respectively. Monomeric $\alpha \mathrm{S}$ thus has a higher affinity for DOPS LUVs than oligomeric $\alpha$ S. Recently, Rhoades et al. (4) have used FCS to quantify the lipid binding of monomeric $\alpha \mathrm{S}$ to LUVs, reporting a dissociation constant of $\sim 2 \mu \mathrm{M}$ for binding of monomeric $\alpha \mathrm{S}$ to POPS LUVs, with a Hill coefficient of $\sim 2.5$. The difference is most likely related to the lipid used. Monomeric $\alpha \mathrm{S}$ has a binding preference for disordered phases (5). At room temperature POPS is close to its phase transition temperature, and therefore the membrane might be sensitive to changes in conditions or the amount of protein bound to the membrane.

Interpreting the difference in the Hill coefficient is not straightforward. In these experiments the amount of protein is kept constant while the binding area (amount of lipids) is changed. Therefore, steeper binding curves $(n>1)$ point to non-cooperative binding while flattening of the binding curves $(n<1)$ points to cooperative binding. This can be shown more intuitively by converting the binding curves of fraction bound versus $f$ total lipid concentration $[L]$, to a plot of the surface concentration of oligomers $\left[P_{s}\right]$ versus the concentration of unbound protein $\left[P_{f}\right]$ (Figure 2$)$. In such a plot the derivative of the slope then 

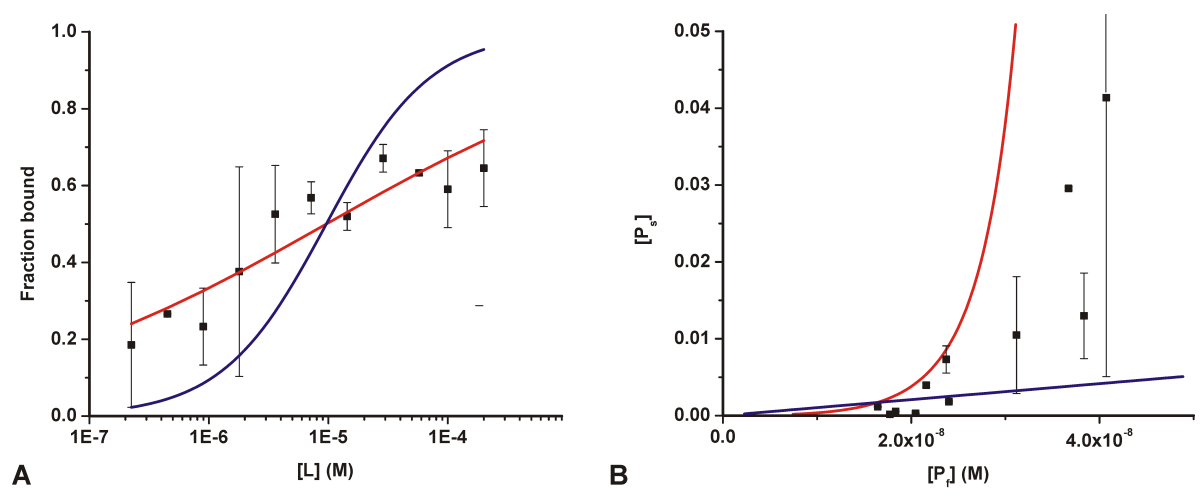

Figure 2. Binding cooperativity (A) Binding curve of $50 \mathrm{~nm}$ labeled $\alpha \mathrm{S}$ oligomers to DOPS LUVs at $25 \mathrm{mM} \mathrm{NaCl}$ (black squares). The red line indicates the Hill fit $\left(K_{d} 1 \mathrm{E}^{-5} \mathrm{M}, n=0.34\right)$ to the data. The blue line is a simulated binding curve according tot the Hill model $\left(K_{d} 1 \mathrm{E}^{-5} \mathrm{M}, n=1\right)$. (B) The binding curves were converted according to equations (2) and (3).

represents the binding affinity (equation 4). If binding is cooperative the lipid affinity increases with the amount of protein on the surface inducing an upwards curvature. Such deviations from the Langmuir isotherm indicate that the lipid affinity of the protein changes with the amount of protein on the membrane. Non-cooperative binding can be indicative of repulsive interactions between proteins on the membrane surface or changes in bilayer packing induced by the protein (6). We have not observed a distinct cooperativity for the binding of monomeric and oligomeric $\alpha \mathrm{S}$ to DOPS LUVs $(n \sim 1)$.

The effect of anionic lipid content on the membrane binding affinity of oligomeric $\alpha \mathrm{S}$ was subsequently quantified. LUVs containing different ratios of the zwitterionic lipid DOPC and the anionic lipid DOPS were prepared. Oligomer binding was strongly dependent on the concentration of anionic lipids in the lipid bilayer (Figure 3A). Lowering the anionic lipid content of the vesicle membrane dramatically reduced the bound fraction of oligomers and the dissociation constant increases. Although the protein has a net negative charge, membrane binding of oligomeric $\alpha \mathrm{S}$ requires a minimum fraction of DOPS in the bilayer, which suggests a role for charge interactions in oligomer membrane binding. These data support our earlier observations that membrane binding and disruption are dependent on the membrane charge (Chapter 3 and 4 ) (7, $8)$.

To further probe the role of electrostatic interactions, the binding of oligomeric $\alpha$ S to DOPS LUVs was measured at different ionic strengths. Binding 

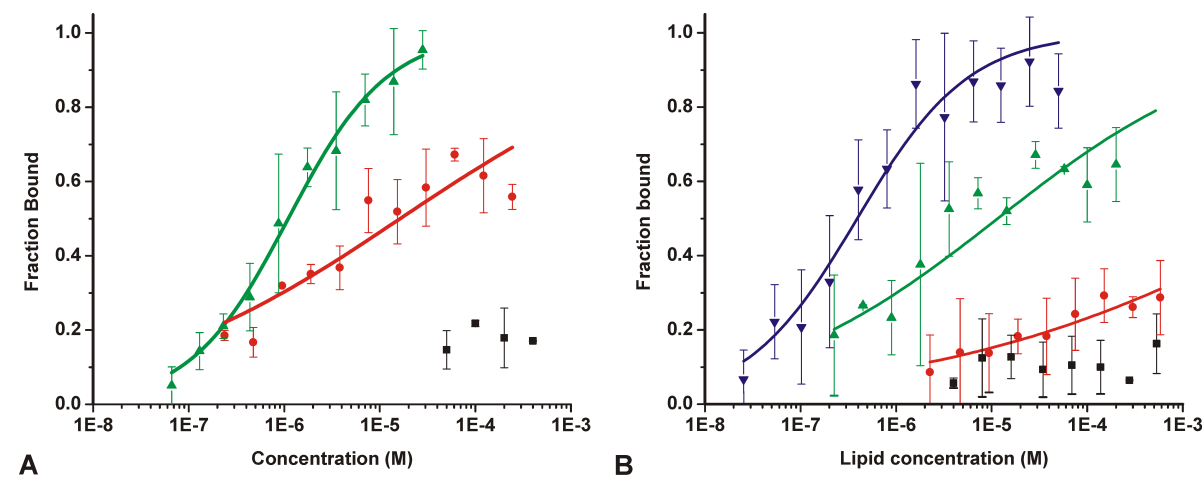

Figure 3. Charge interaction (A) Binding curves of $50 \mathrm{nM}$ oligomeric $\alpha \mathrm{S}$ to LUVs composed of different ratios of DOPC to DOPS lipids; $20 \%$ DOPS (black squares), $60 \%$ DOPS (red circles) and $100 \%$ DOPS (green triangles). (B) Binding curves of $50 \mathrm{nM}$ oligomeric $\alpha \mathrm{S}$ to DOPS LUVs at different $\mathrm{NaCl}$ concentrations: 0 $\mathrm{mM}$ (black squares), $10 \mathrm{mM}$ (red circles), $25 \mathrm{mM}$ (green triangles), $50 \mathrm{mM}$ (blue inverted triangles).

was strongest at $50 \mathrm{mM} \mathrm{NaCl}$, and at low ionic strengths, oligomer lipid binding was almost completely inhibited (Figure 3B). Fitting the Hill equation to the binding curves shows an increase in cooperativity and dissociation constant upon decreasing salt concentration. This is most likely caused by an electrostatic repulsion between the negatively charged protein and the anionic lipids.

Interpreting the binding curves at different lipid compositions and ionic strengths is challenging. The observed increase in binding cooperativity at low ionic strength and lower charge densities may point to aggregation of the oligomers or alterations in bilayer structure. $\alpha \mathrm{S}$ oligomeric species might be heterogeneous and are known to affect bilayer integrity. Also conformational changes in the $\alpha \mathrm{S}$ oligomers upon membrane binding or under different solution conditions cannot be excluded. In addition, existing binding models of the role of electrostatics in protein lipid binding only consider the binding of positively charged proteins to negatively charged membranes. Therefore, pinpointing what actually causes these changes in the binding curves is difficult.

The specific binding of monomeric $\alpha \mathrm{S}$ to anionic lipids is well understood. The interaction is mainly electrostatic in nature (4). At neutral $\mathrm{pH}, \alpha \mathrm{S}$ has a net negative charge. However, the lipid binding region contains multiple positively charged and hydrophobic residues, explaining the lipid binding characteristics. The C-terminus, which contains most of the negatively charged residues, remains unstructured in solution (9). The structural characteristics 
of $\alpha \mathrm{S}$ oligomers are unknown and which residues are involved in lipid binding is not clear.

The membrane binding of oligomeric $\alpha \mathrm{S}$ is facilitated by the presence of anionic DOPS in the membrane. The specific binding of the net negatively charged oligomers to anionic lipids suggests that lipid binding involves the positively charged N-terminus. However, similar to the monomer, the charge distribution in the oligomeric complex must be such that the negative charges in C-terminus are not close to the membrane. The oligomers must therefore contain structural features such as positively charged patches, or an ability to reorganize upon membrane binding to maximize favorable charge interactions. In an oligomeric aggregate, the individual monomers are not likely to show a great conformational flexibility, so a reorganization upon binding might be restricted. Therefore, there could be an energy penalty for bringing the Cterminus close to the membrane upon oligomer lipid binding. This hypothesis also can explain the lower lipid affinity compared to the monomeric form of the protein. This penalty will be larger at low ionic strengths, which explains the observation that binding of $\alpha \mathrm{S}$ oligomers to the membrane is inhibited under these conditions.

Both circular dichroism and Raman spectroscopy have shown that oligomers have a considerable secondary structure $(10,11)$. Based on these results and our data we propose that oligomers possess distinct structural features such as positively charged patches on the oligomer surface which explain their lipid binding characteristics.

\section{Materials and Methods}

Preparation of monomeric and oligomeric $\alpha S$. Preparation of monomeric wild type $\alpha \mathrm{S}$, the cysteine to alanine mutant $\alpha \mathrm{S}-\mathrm{A} 140 \mathrm{C}$, labeling of $\alpha \mathrm{S}-\mathrm{A} 140 \mathrm{C}$ using Alexa 488 C5-maleimide (Invitrogen) and preparation of the labeled oligomers were performed as described in Chapter 2.

Preparation of vesicles. DOPC and DOPS were obtained from Avanti Polar lipids. Vesicles of various compositions were prepared by mixing the lipids from stock solutions in chloroform. Approximately $1 \mathrm{mg}$ of lipid was deposited in a glass vial and dried using nitrogen gas. Residual chloroform was removed by drying under vacuum for 4 hours. The lipid film was hydrated for 1 hour at room temperature with regular vortexing in $10 \mathrm{mM}$ HEPES, pH 7.4, $50 \mathrm{mM}$ $\mathrm{NaCl}$ unless noted otherwise. LUVs were obtained by extrusion through a filter membrane with a $100 \mathrm{~nm}$ pore size. The lipid concentration was subsequently measured using the protocol of Chen et al. (12).

Sample preparation. Cover glasses were treated to prevent adsorption of the protein to the surface. After cleaning in piranha solution for 15 minutes 
Quantifying oligomer-lipid linding

(3:1 ratio of concentrated sulfuric acid to $30 \%$ hydrogen peroxide solution) and rinsing with ultrapure water, the slides were pacified with a PEG-silane compound. Coverglasses were incubated overnight in $0.1 \%$ weight/volume 2-[Methoxy (polyethyleneoxy) propyl] trimethoxy silane (ABCR) in toluene under a nitrogen atmosphere. After this procedure the cover glasses were rinsed with fresh toluene and subjected to a 1 minute sonication. Before measuring the correlation curves $\alpha \mathrm{S}$-A140C-AL488 monomers or labeled oligomeric $\alpha \mathrm{S}$ were added to a specified concentration of LUVs, to a final protein concentration of $50 \mathrm{nM}$ (equivalent monomer concentration for the oligomers) and incubated for 15 minutes on the cover slip under a humid atmosphere.

Fluorescence correlation spectroscopy. FCS was performed as described in Appendix A.

Data analysis. Analysis of the correlation curves was performed as described in Appendix A. The resulting binding curves were fit with the Hill equation:

$$
f=\frac{[L]^{n}}{K_{d}+[L]^{n}}
$$

where $[L]$ is the lipid concentration, $K_{d}$ is the dissociation constant and $n$ is a cooperativity coefficient. This equation is generally used for fitting of binding curves where the concentration of ligand is varied and the amount of binding spots in constant. In our experiments the protein concentration was constant and the amount of binding surface (lipid concentration) was varied. In that case $n<1$ binding indicates cooperative binding, whereas $n>1$ indicates a negative cooperativity. This can be shown more intuitively by converting the binding curves of fraction bound versus $f$ total lipid concentration $[L]$ to a plot of the surface concentration of oligomers $\left[P_{s}\right]$ versus the unbound concentration of oligomers $\left[P_{f}\right]$. Where,

$$
\left[P_{s}\right]=\frac{f\left[P_{t o t}\right]}{[L]}
$$

and

$$
\left[P_{f}\right]=(1-f)\left[P_{t o t}\right]
$$

In such a plot the initial slope is determined by the affinity constant or partition coefficient $\mathrm{K}$ :

$$
\left[P_{s}\right]=K\left[P_{f}\right]
$$

For cooperative binding the affinity for the protein changes with the amount of protein present on the membrane. A cooperative interaction on the membrane surface will lead to an upward curvature in the binding curves (6), as is shown in Figure 3. 


\section{References}

(1) Spillantini, M. G., Schmidt, M. L., Lee, V. M., Trojanowski, J. Q., Jakes, R., and Goedert, M. (1997) Alpha-synuclein in Lewy bodies. Nature 388, 839-840.

(2) Lee, V. M., and Trojanowski, J. Q. (2006) Mechanisms of Parkinson's disease linked to pathological alpha-synuclein: new targets for drug discovery. Neuron 52, 33-38.

(3) Lashuel, H. A., Hartley, D., Petre, B. M., Walz, T., and Lansbury, P. T., Jr. (2002) Neurodegenerative disease: amyloid pores from pathogenic mutations. Nature 418, 291.

(4) Rhoades, E., Ramlall, T. F., Webb, W. W., and Eliezer, D. (2006) Quantification of alpha-synuclein binding to lipid vesicles using fluorescence correlation spectroscopy. Biophys. J. 90, 4692-4700.

(5) Stöckl, M., Fischer, P., Wanker, E., and Herrmann, A. (2008) Alpha-synuclein selectively binds to anionic phospholipids embedded in liquid-disordered domains. J. Mol. Biol. 375, 1394-1404.

(6) Hinderliter, A., and May, S. (2006) Cooperative adsorption of proteins onto lipid membranes. J. Phys. Condens. Matt. 18, S1257-S1270.

(7) van Rooijen, B. D., Claessens, M. M. A. E., and Subramaniam, V. (2008) Membrane binding of oligomeric alpha-synuclein depends on bilayer charge and packing. FEBS Lett. 582, 3788-3792.

(8) van Rooijen, B. D., Claessens, M. M. A. E., and Subramaniam, V. (2009) Lipid bilayer disruption by oligomeric alpha-synuclein depends on bilayer charge and accessibility of the hydrophobic core. Biochim. Biophys. Acta-Biomembr. 1788, 1271-1278.

(9) Eliezer, D., Kutluay, E., Bussell, R., Jr., and Browne, G. (2001) Conformational properties of alpha-synuclein in its free and lipidassociated states. J. Mol. Biol. 307, 1061-1073.

(10) Volles, M. J., Lee, S. J., Rochet, J. C., Shtilerman, M. D., Ding, T. T., Kessler, J. C., and Lansbury, P. T., Jr. (2001) Vesicle permeabilization by protofibrillar alpha-synuclein: implications for the pathogenesis and treatment of Parkinson's disease. Biochemistry 40, 7812-7819.

(11) Apetri, M. M., Maiti, N. C., Zagorski, M. G., Carey, P. R., and Anderson, V. E. (2006) Secondary structure of alpha-synuclein oligomers: characterization by raman and atomic force microscopy. J. Mol. Biol. 355, 63-71.

(12) Chen, P. S., Toribara, T. Y., and Warner, H. (1956) Microdetermination of Phosphorus. Anal. Chem. 28, 1756-1758. 


\section{Chapter 6 Tryptophan fluorescence reveals structural features of $\alpha$-synuclein oligomers}

Oligomeric $\alpha$-synuclein is considered to be the potential toxic species responsible for the onset and progression of Parkinson's disease, possibly through the disruption of lipid membranes. Although there is evidence that oligomers contain considerable amounts of secondary structure, more detailed information on the structural characteristics and how these mediate oligomer-lipid binding are critically lacking. In this chapter we aim to address the structure of oligomeric $\alpha$-synuclein on a more detailed level. We have used tryptophan fluorescence spectroscopy to gain insight into the structural features of oligomeric $\alpha$-synuclein and the structural basis for oligomer-lipid interactions. Several single tryptophan mutants of $\alpha$-synuclein were used to gain site-specific information about the microenvironment of monomeric, oligomeric and lipid bound oligomeric $\alpha$-synuclein. Acrylamide quenching and spectral analyses indicate that the tryptophan residues are considerably more solvent protected in the oligomeric form compared to the monomeric protein. In the oligomers, the negatively charged C-terminus was the most solvent exposed part of the protein. Upon lipid binding a blue shift in fluorescence is observed for $\alpha$-synuclein mutants where the tryptophan is located within the N-terminal region. These results suggest that as in the case of monomeric $\alpha$-synuclein, the N-terminus is critical in determining oligomer-lipid binding. 


\section{Introduction}

A possible mechanism of toxicity of $\alpha \mathrm{S}$ oligomers, is through the permeabilization of cellular membranes $(1,2)$. However, the topic of oligomer induced toxicity remains controversial. Structural information about oligomeric species is critically lacking and the structural basis for oligomer-lipid interaction and toxicity is not understood. In addition to its importance to toxicity, the structure of oligomeric intermediates is important for understanding the aggregation process. Aggregation of $\alpha \mathrm{S}$ is believed to occur through a process of nucleation dependent polymerization (3). However, the nature of this nucleus and how it is formed is unknown. Initial data show that oligomers are not randomly collapsed aggregates but contain significant amounts of secondary structure. Oligomers have been reported to contain considerable $\beta$-sheet structure (4) although $\alpha$-helical content has also been observed (5).

Several fundamental problems limit the determination of the structure of $\alpha \mathrm{S}$ oligomers. Early intermediates can be unstable and in fast equilibrium with the monomeric protein (6). Aggregation intermediates are transient in nature and disappear upon fibril formation (7). Preparation of stable oligomers generally results in low yields and thus only little material is available (4). Finally, oligomers are possibly heterogeneous in size, structure and function $(6,8)$. Thus, determining the exact structure of all possible oligomers of $\alpha \mathrm{S}$ remains a challenging task. However, the structure of the different oligomeric species and the fibrillar structure might be related since the driving force of the aggregation is the same for all species. Therefore, establishing structural constraints for the $\alpha \mathrm{S}$ oligomeric species should be feasible.

In order to overcome some of these challenges and to gain insights into oligomer structure and the structural basis for its interaction with lipids, we have used tryptophan (Trp) fluorescence spectroscopy on stable purified $\alpha \mathrm{S}$ oligomers. These oligomers occur during $\alpha \mathrm{S}$ aggregation and have been previously used to study oligomer-lipid interactions $(4,9)$. Several single Trp mutants of $\alpha \mathrm{S}$ were generated to assess the microenvironment of different regions of the protein in the monomeric, oligomeric and lipid bound oligomeric state. Here we show how individual monomers might be arranged in an oligomeric aggregate and what regions of the $\alpha \mathrm{S}$ molecule mediate the lipid interaction in the oligomeric form.

The results from acrylamide quenching experiments and spectral analyses indicate that, compared to the monomeric protein, the engineered Trp residues are more solvent protected in the oligomeric form. The negatively charged C-terminus was the most solvent exposed part of the oligomers. When lipid vesicles were added to oligomeric $\alpha \mathrm{S}$ a blue shift in fluorescence is observed for $\alpha \mathrm{S}$ mutants where the tryptophan is located within the N-terminal region of 


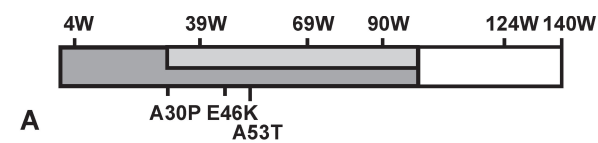

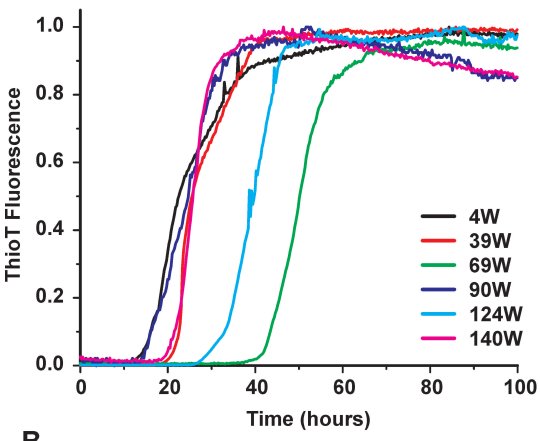

B

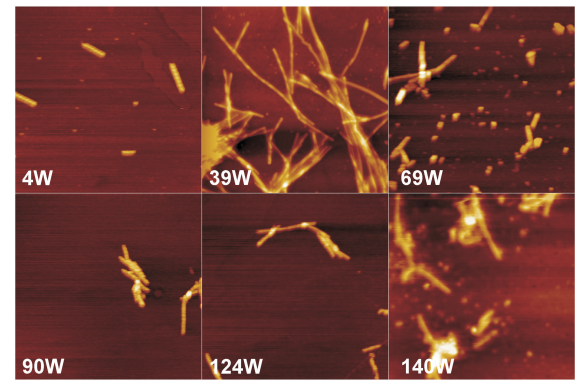

C

Figure 1. Aggregation properties (A) Mutants containing a Trp residue in different regions of the protein were created. The N-terminal residues $\sim 1-100$ (dark gray) are involved in lipid binding of monomeric $\alpha \mathrm{S}$, residues $\sim 35-100$ (light grey) make up the core of fibrillar $\alpha \mathrm{S}$ and the C-terminus $\sim 100-140$ (white) remains unstructured under most conditions. The disease related mutations are also shown in the figure. (B) Aggregations of the different $\alpha \mathrm{S}$ Trp followed by the dye ThioT. The ThioT fluorescence signal of the different Trp mutants all showed a rapid increase of fluorescence after a certain lag time, which is typical for amyloid formation. (C) AFM images of fibrils formed from the different $\alpha \mathrm{S}$ Trp mutants.

residues 4 to 90 . This observation indicates that, similar to the monomeric protein, the N-terminal part of the protein most likely mediates the lipid binding of $\alpha \mathrm{S}$ oligomers.

\section{Results}

Since $\alpha$ S-wt does not contain any Trp residues, replacing an amino acid in the $\alpha \mathrm{S}$ sequence with a Trp results in site-specific information about the microenvironment. A number of mutants containing a single Trp substitution in different regions of the protein were prepared using site-directed mutagenesis (F4W, Y39W, A69W, A90W, A124W and A140W) (Figure 1A). We performed aggregation experiments to check if the introduced mutations in $\alpha \mathrm{S}$ affected fibril formation. All $\alpha \mathrm{S}$ Trp mutants showed typical sigmoidal growth curves characteristic of fibril formation when monitored using a ThioT fluorescence assay (Figure 1B). Fibril formation was further confirmed by AFM imaging 

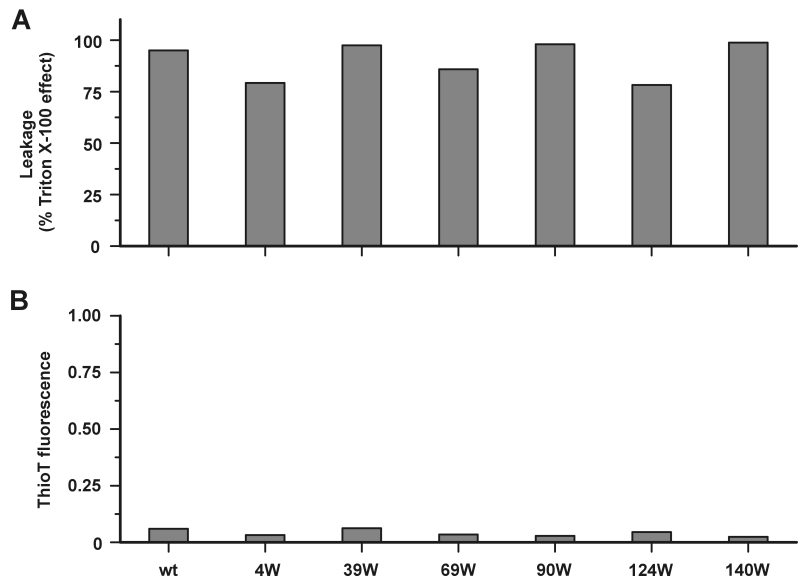

Figure 2. Oligomer characterization Properties of oligomers of the $\alpha \mathrm{S}$ Trpmutants compared to those of $\alpha \mathrm{S}$-wt (A) Oligomers of the different $\alpha \mathrm{S}$ Trp mutants were characterized using a dye efflux assay on DOPS LUVs at final concentrations of $0.5 \mu \mathrm{M} \alpha \mathrm{S}$ and $20 \mu \mathrm{M}$ lipids. The oligomeric species formed from the Trp-mutants were able to disrupt the LUVs to similar extents as wt oligomers. (B) The bars show the ThioT fluorescence induced by $0.5 \mu \mathrm{M}$ oligomeric $\alpha \mathrm{S}$ relative to that measured in the presence of $0.5 \mu \mathrm{M}$ fibrillar $\alpha \mathrm{S}$.

(Figure 1C).

Oligomeric mutant $\alpha \mathrm{S}$ was prepared following the same protocol as for $\alpha$ S-wt. The resulting Trp containing oligomers possessed similar properties as those prepared from $\alpha$ S-wt. The oligomers induced membrane disruption when added to LUVs and did not induce a considerable increase in fluorescence in a ThioT fluorescence assay (Figure 2).

The emission wavelength of Trp fluorescence is sensitive to the polarity of the environment. Spectral analysis of the Trp emission from proteins therefore reports on the properties of the microenvironment of the corresponding Trp residues. Solvent exposed Trp residues show a red shift in the fluorescence compared to buried Trp residues in proteins. The Trp emission spectrum was recorded for the different mutants of $\alpha \mathrm{S}$ in the monomeric and oligomeric form. In addition, spectra of oligomers in the presence of DOPS LUVs were recorded. We have previously reported that oligomeric $\alpha \mathrm{S}$ can bind and permeabilize vesicles composed of DOPS $(9,10)$.

The emission peak wavelengths were determined from the emission spectra (Figure 3A) by fitting equation 1 (11) (see Materials and Methods). The resulting peak wavelengths for monomeric and oligomeric $\alpha \mathrm{S}$ are plotted in Figure 

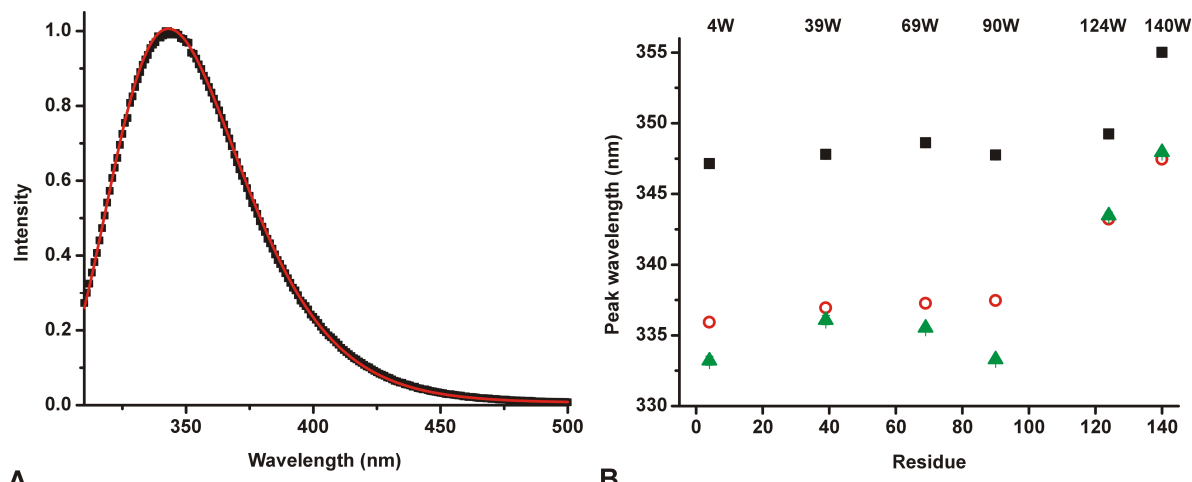

A

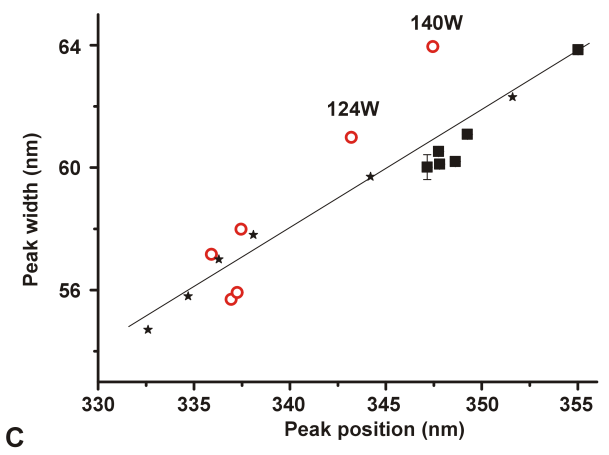

Figure 3. Emission wavelength (A) Tryptophan emission spectrum of oligomeric $\alpha \mathrm{S}-124 \mathrm{~W}$. The spectrum is fitted with a log-normal function which estimates the peak emission wavelength and the spectral width at half maximum. (B) The peak wavelength of the Trp emission plotted for monomeric $\alpha \mathrm{S}$ (squares), oligomeric $\alpha \mathrm{S}$ (open circles) and lipid bound oligomers (triangles), as a function of the position of the inserted Trp. (C) The width of the emission spectrum plotted as a function of the peak wavelength for monomeric (squares) and oligomeric (open circles) $\alpha \mathrm{S}$. Data from the spectral analysis of NATA in solvents of different polarity (stars) and a linear fit through these points are also plotted. The error bars indicate the standard deviation $(n=2)$. 
3B and summarized in Table 1. For monomeric $\alpha \mathrm{S}$ the Trp emission maximum wavelengths were between 347 to $349 \mathrm{~nm}$ for the $4 \mathrm{~W}, 39 \mathrm{~W}, 69 \mathrm{~W}, 90 \mathrm{~W}$ and $124 \mathrm{~W} \alpha \mathrm{S}$ mutants and around $355 \mathrm{~nm}$ for $\alpha \mathrm{S}-140 \mathrm{~W}$. Emission at these wavelengths is indicative of a relatively polar environment for all the monomeric Trp mutants, as can be expected for an intrinsically disordered protein. In the oligomeric form, the emission from Trp mutants $4 \mathrm{~W}, 39 \mathrm{~W}, 69 \mathrm{~W}$ and $90 \mathrm{~W}$ was considerably blue-shifted with emission maxima of 336 to $338 \mathrm{~nm}$. These residues are thus significantly less exposed to the solvent and buried in the aggregate. The blue shift was less pronounced for $\alpha \mathrm{S}-124 \mathrm{~W}$ and $\alpha \mathrm{S}-140 \mathrm{~W}$, which showed emission maxima at 343 and $348 \mathrm{~nm}$ respectively. It thus appears that the highly negatively charged C-terminus of the protein remains more solvent exposed, and that the residues 4-90 make up the core of the oligomeric aggregate. When DOPS LUVs are added to the oligomers, the Trp emission spectra of the mutants $4 \mathrm{~W}, 69 \mathrm{~W}$ and $90 \mathrm{~W}$ were even more blue shifted. This blue shift was less pronounced for $\alpha$ S-39WS. The spectra of the $124 \mathrm{~W}$ and $140 \mathrm{~W}$ mutants remained unchanged. The blue shift upon lipid interaction indicates that these residues might embed in the hydrophobic core of the membrane, or that the N-terminus changes its conformation upon membrane binding (12).

In addition to the peak wavelength, the width of the emission spectrum also carries information. In homogeneous solvents the width of the emission spectra depends on the emission wavelength (11). In heterogeneous emitting systems, where different populations of Trp residues sense a different environment, spectral broadening does occur. This can be visualized by plotting the emission width as a function of peak wavelength. A broadened spectrum compared to the N-acetyl-L-tryptophanamide (NATA) emission in different solvents is a measure of emitter heterogeneity. As can be seen in Figure 3C, spectral broadening does not occur for monomeric $\alpha \mathrm{S}$. For the oligomeric protein most mutants do not show signs of spectral broadening. Only the spectra of the $124 \mathrm{~W}$ and $140 \mathrm{~W}$ mutants show considerable deviations from NATA emission in pure solvents.

Quenching of Trp fluorescence by added solutes is another powerful method to measure the accessibility of Trp residues (13). Since we have observed in Chapter 5 that the membrane interaction of oligomeric $\alpha \mathrm{S}$ is dependent on the salt concentration (10), we have chosen acrylamide as a quenching agent instead of iodide ions. The Trp fluorescence quenching by acrylamide is mainly a collisional effect and is thus dependent on the concentration of the quencher and the solvent accessibility of the Trp residue. Figure 4A shows an example of a typical quenching experiment, where a spectrum is recorded after each addition of acrylamide. Stern-Volmer plots were generated from these spectra (Figure 4B). For monomeric and oligomeric $\alpha \mathrm{S}$, the Stern-Volmer plots showed an upwards curvature. Therefore these were fitted with a modified form of 

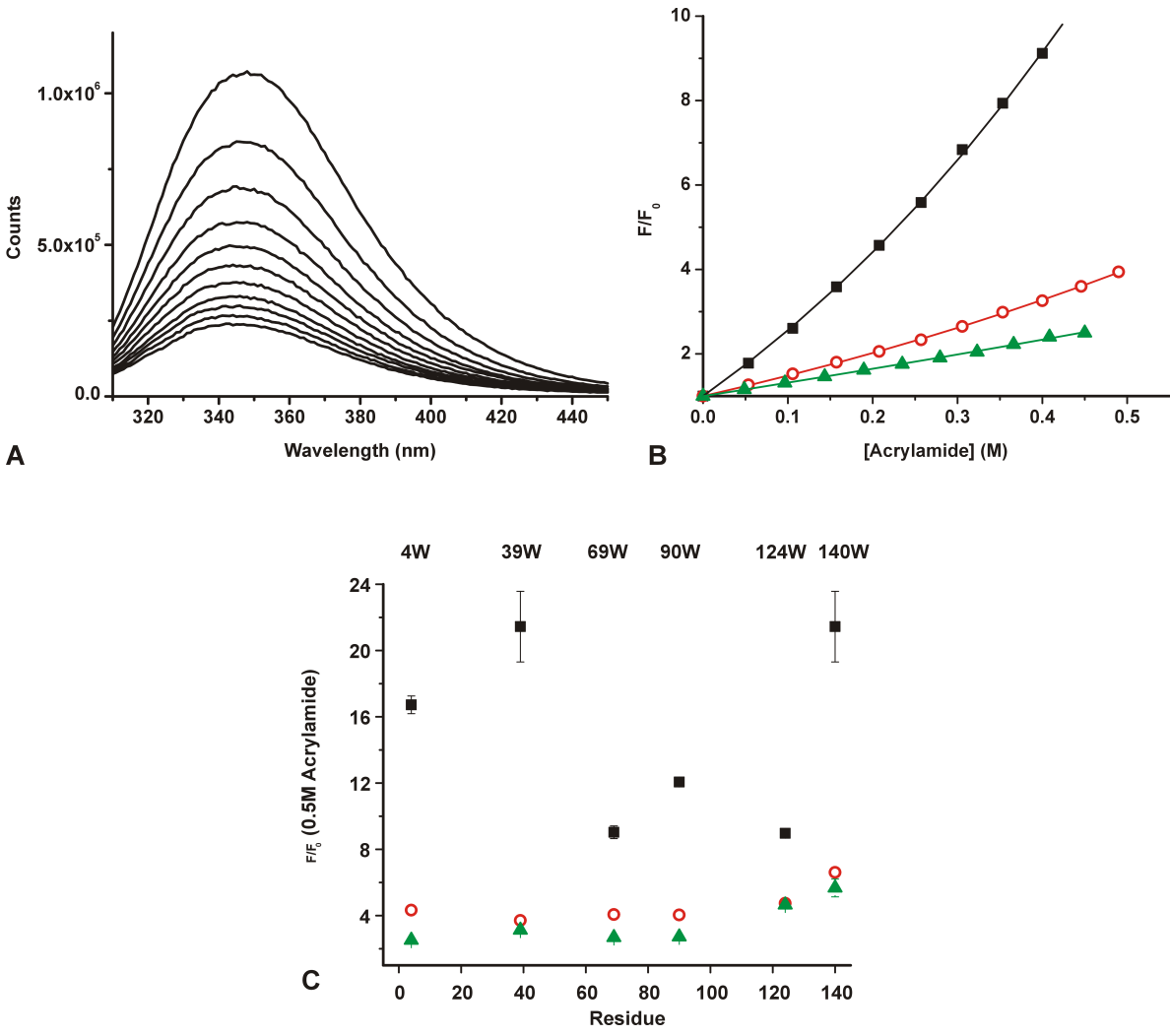

Figure 4. Tryptophan quenching (A) Quenching of Trp emission of oligomeric $\alpha \mathrm{S}-90 \mathrm{~W}$ by addition of increasing amounts of acrylamide. (B) Stern-Volmer plots of monomeric (squares), oligomeric (open circles) and lipid bound oligomeric (triangles) $\alpha \mathrm{S}-90 \mathrm{~W}$. The data were fitted using the modified Stern-Volmer equation (eq.2). (C) A plot of the calculated quenching ratio at $0.5 \mathrm{M}$ acrylamide from the fit results (Table 1) as a function of the position of the inserted Trp for monomeric (squares), oligomeric (open circles) and lipid bound oligomeric (triangles) $\alpha \mathrm{S}$. The error bars indicate the standard deviation $(n=2)$. 

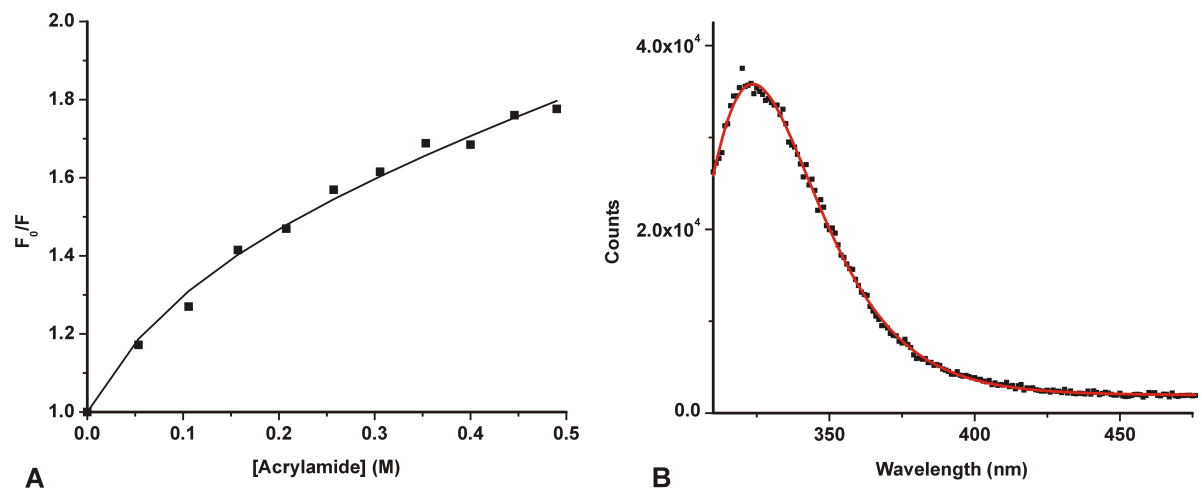

Figure 5. Fibrillar $\alpha \mathbf{S}$ (A) Stern-Volmer plot of fibrillar $\alpha$ S-69W. Residual monomeric $\alpha \mathrm{S}$ contributes to the fluorescence signal therefore a two component fit was used to describe the experimental data. (B) Trp emission spectrum of $\alpha \mathrm{S}-69 \mathrm{~W}$ fibrils, with an emission maximum at $\sim 324 \mathrm{~nm}$.

the Stern-Volmer equation (equation 2) that also accounts for static quenching (13). The quenching experiments were performed for all $\alpha \mathrm{S}$ Trp mutants.

The results of fitting the Stern-Volmer plots are summarized in Table 1. Since the curvature of the Stern-Volmer plots might also be caused by a heterogeneity of the position of the Trp residue in the oligomer, the Stern-Volmer constants derived from the fitting procedure were not directly used for comparison of the different Trp mutants. To allow an unbiased comparison among the different Trp mutants, the fit results were rather used to calculate the quenching ratio at $0.5 \mathrm{M}$ acrylamide as a general parameter of accessibility (Figure 4C). For monomeric $\alpha \mathrm{S}$ the Trp fluorescence can be easily quenched especially at $\mathrm{N}$ - and C-termini of the protein ( $4 \mathrm{~W}$ and $140 \mathrm{~W})$. In the oligomeric form, the Trp residues in the mutants $4 \mathrm{~W}, 39 \mathrm{~W}, 69 \mathrm{~W}$, and $90 \mathrm{~W}$ are considerably more protected, which is in agreement with the observed blue shift in emission. Compared to monomeric $\alpha \mathrm{S}$, the oligomers from the $124 \mathrm{~W}$ and $140 \mathrm{~W}$ Trp mutants were more protected from quenching by acrylamide. However consistent with the view from the spectral analysis that the C-terminus of $\alpha \mathrm{S}$ is solvent exposed, the oligomers from the $124 \mathrm{~W}$ and $140 \mathrm{~W}$ mutant showed the highest Stern-Volmer constants.

To compare the structural properties of oligomers to those of fibrils, Trp fluorescence spectroscopy and quenching experiments were also performed on fibrils prepared form the different Trp mutants. However, the results were generally of poor quality. Around 5 to $10 \%$ of soluble $\alpha \mathrm{S}$ (as assayed by the optical absorbance of the supernatant after centrifugation) was still present 
after a week of aggregation. In addition, fibril containing solutions were turbid and larger particles sank to the bottom of the cuvette. However, the results from the $69 \mathrm{~W}$ mutant highlight that the residues in the hydrophobic amyloidogenic part of the protein are buried. The Trp emission was highly protected from quenching by acrylamide (Figure 6). Furthermore, the emission peak wavelength was shifted to around $324 \mathrm{~nm}$. In the densely packed amyloid core of the fibrils, the residues are thus considerably more protected compared to the oligomers.

\section{Discussion}

Elucidating the conformation of intermediates in the aggregation of $\alpha \mathrm{S}$ remains an important question that is still unresolved. Although oligomeric $\alpha \mathrm{S}$ is known to contain secondary structural elements $(4,5)$, more detailed information on how individual monomers are arranged in the aggregate is not available. Trp fluorescence is an extremely useful tool to learn about protein structure and has successfully been used to monitor $\alpha \mathrm{S}$ conformational changes $(7,14-16)$. The data presented here allow a unique view on how individual monomers might be arranged in an oligomeric aggregate.

The $\alpha S$ Trp mutants used in this study formed amyloid fibrils and oligomers prepared from these mutants had similar properties as those prepared from $\alpha \mathrm{S}$ wt. We therefore conclude that the inserted Trp residues did not significantly affect $\alpha \mathrm{S}$ conformational properties. Monomeric $\alpha \mathrm{S}$ has the characteristics of an intrinsically disordered protein (17). In solution the protein has no defined secondary and tertiary structure. Although the Stokes radius is larger compared to a globular protein of similar weight, the protein is most likely not fully extended but rather partially collapsed (18). Long range interactions between the N- and C-terminus of the protein have been reported (19). Our results support this view of the $\alpha \mathrm{S}$ monomer. The Trp emission maxima of around 348-349 nm for mutants 4W, 39W, 69W, 90W and $124 \mathrm{~W}$ and around $355 \mathrm{~nm}$ for $140 \mathrm{~W}$, point to a polar environment. Generally the emission wavelengths are slightly blue shifted compared to free NATA in water ( $\lambda_{\max } 355$ $\mathrm{nm}$ ). Since the Trp emission wavelength can also be influenced by neighboring residues (12), acrylamide quenching studies give additional information. Trp fluorescence was efficiently quenched with typical Stern-Volmer constants of around 10-20. Thus for monomeric $\alpha \mathrm{S}$ all residues are considerably solvent exposed, which is consistent with other reports (20).

The aggregation of $\alpha \mathrm{S}$ is driven by an hydrophobic stretch of amino acids in the middle of the protein sequence (21). Although the exact fibril structure of $\alpha \mathrm{S}$ is not yet resolved, it is generally considered that residues $\sim 35$-100 form the amyloid core of the fibril $(20,22)$. This core is densely packed and protected 


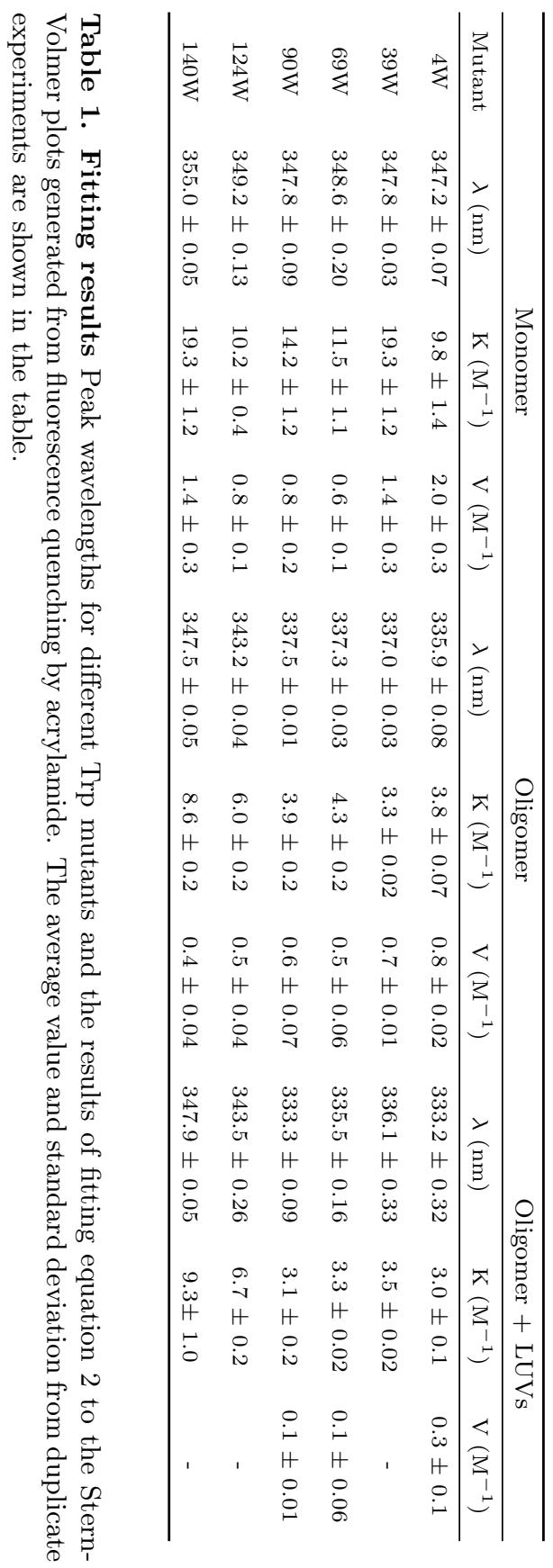


from proteolysis (23) and hydrogen-deuterium exchange (20). How the structure of the oligomeric species is related to the fibrillar structure is currently not known. Our data show that especially the residues in the N-terminus and in the hydrophobic core (mutants $4 \mathrm{~W}, 39 \mathrm{~W}, 69 \mathrm{~W}$ and $90 \mathrm{~W}$ ) sense a considerably more apolar and protected environment compared to the monomer. The peak wavelength of the Trp emission for these residues is blue shifted to around 336-338 nm. In addition, quenching by acrylamide is reduced to Stern-Volmer constants of typically around 4 . However, these values differ from completely buried Trp residues in proteins $(24,25)$. We have observed significantly larger blue shifts and smaller Stern-Volmer constants from fibrils from Trp-mutant $\alpha \mathrm{S}-69 \mathrm{~W}$. Thus, compared to $\alpha \mathrm{S}$ fibrils, oligomers are most likely less densely packed. The C-terminus appears to be the part of the protein that is most exposed to the solvent for oligomeric $\alpha \mathrm{S}$. The emission wavelength showed a smaller blue shift and the Stern-Volmer constants were higher compared to the other mutants. The fact that the C-terminus remains relatively exposed is consistent with it being highly soluble and with containing many negatively charged residues.

The interaction of oligomeric $\alpha \mathrm{S}$ with membranes has been suggested as a possible mechanism of toxicity (1). Permeabilization might occur through a pore-like mechanism $(4,26)$, although the interaction is still not well understood. The membrane binding of monomeric $\alpha \mathrm{S}$ has been much better characterized. Monomeric $\alpha \mathrm{S}$ specifically binds negatively charged lipids (27). The N-terminal residues $\sim 1-100$ participate in lipid binding and fold into an $\alpha$-helical conformation (28). We have shown in Chapter 4 and 5 that oligomeric $\alpha \mathrm{S}$ also specifically binds to negatively charged membranes (9). This implies that the lipid binding of oligomeric $\alpha \mathrm{S}$ possibly involves the same N-terminal residues. The data presented here support this idea.

Upon addition of DOPS LUVs to oligomeric $\alpha \mathrm{S}$, we observed a pronounced blue shift in the Trp emission spectra of the $4 \mathrm{~W}, 69 \mathrm{~W}$ and $90 \mathrm{~W}$ mutants. This blue shift could be the result of a direct interaction with the hydrophobic core of the membrane. Alternatively, a conformational change in the $\alpha \mathrm{S}$ oligomer could also result in the change in Trp emission. However, both explanations point to changes in the N-terminus upon the interaction of oligomeric $\alpha \mathrm{S}$ with lipids. The $39 \mathrm{~W}$ mutant showed a less pronounced blue shift. The reason for this is unclear; for instance there are no highly charged residues in this part of the protein which would prevent this region from interacting with the bilayer interior. We note that this position is located directly next to a break in the $\alpha$-helical lipid binding motif of the monomeric protein (29). The C-terminal mutants $124 \mathrm{~W}$ and $140 \mathrm{~W}$ did not show a blue shift. The high negative charge of the C-terminus might prevent this part of the protein from interacting with the negatively charged membrane. Quenching by acrylamide is generally slightly 
reduced in the presence of DOPS LUVs and the C-terminus remains the most solvent exposed part of the protein.

In summary, the data presented here offer site specific information on the conformational characteristics of $\alpha \mathrm{S}$ oligomers. From our data we conclude that in the oligomeric form of $\alpha \mathrm{S}$, residues 4-90 make up the core of the aggregate while the C-terminus is most solvent exposed. In addition, we propose that lipid binding of oligomeric $\alpha \mathrm{S}$ is mediated by the N-terminus.

\section{Materials and Methods}

Purification of $\alpha S$ and preparation of oligomers. The following mutants of $\alpha \mathrm{S}$ with single tryptophan amino acid substitutions were generated by site-directed mutagenesis: Phenylalanine to Trp at position $4(\alpha \mathrm{S}-4 \mathrm{~W})$, Tyrosine to Trp at $39(\alpha \mathrm{S}-39 \mathrm{~W})$, Alanine to Trp at position $69(\alpha \mathrm{S}-69 \mathrm{~W})$, Alanine to Trp at position $90(\alpha \mathrm{S}-90 \mathrm{~W})$, Alanine to Trp at position $124(\alpha \mathrm{S}-124 \mathrm{~W})$ and Alanine to Trp at position $140(\alpha \mathrm{S}-140 \mathrm{~W})$. Purification of mutant $\alpha \mathrm{S}$ and preparation of the oligomeric species were performed similar to $\alpha \mathrm{S}$-wt as was described in Chapter 2.

Aggregation assay. Aggregations of the different $\alpha \mathrm{S}$ Trp mutants were performed in a Tecan Safire platereader. The protein was incubated in a 96 well plate at $37^{\circ} \mathrm{C}$ with orbital shaking, at a concentration of $100 \mu \mathrm{M}$ in $10 \mathrm{mM}$ Hepes $\mathrm{pH} 7.4$ and $50 \mathrm{mM} \mathrm{NaCl}$ in the presence of $40 \mu \mathrm{M}$ of the dye ThioT.

Vesicle permeabilization. The vesicle permeabilization assay was performed similar as described in Chapter 3.

Atomic force micoscopy. AFM was performed as described in Chapter 2.

Preparation of LUVs. To prepare LUVs, $2 \mathrm{mg}$ of DOPS from a stock solution in chloroform was deposited in a glass vial. The lipids were dried using a gentle stream of nitrogen gas. Residual chloroform was removed by drying the lipid film in a vacuum for at least 4 hours. The lipid film was hydrated by the addition of a solution of $10 \mathrm{mM}$ HEPES, pH 7.4, $150 \mathrm{mM} \mathrm{NaCl}$. LUVs were subsequently obtained by extrusion through a polycarbonate filter membrane with a pore size of $100 \mathrm{~nm}$.

Fluorescence spectroscopy. Fluorescence spectra were recorded on a Jobin Yvon Fluoromax 4 fluorimeter. The excitation wavelength was set at $295 \mathrm{~nm}$ to prevent excitation of the Tyrosine residues of $\alpha \mathrm{S}$. Fluorescence emission was recorded from 310 to $500 \mathrm{~nm}$. The slit width was set to $5 \mathrm{~nm}$ for both the excitation and emission. Fluorescence spectra were obtained at a protein concentration of around $2 \mu \mathrm{M}$. For the quenching experiments, acrylamide from a $40 \%$ stock solution (weight/volume) was added in $5 \mu \mathrm{l}$ aliquots, to $500 \mu \mathrm{l}$ of protein solution. After each addition a spectrum was recorded. For the measurements in the presence of LUVs, polarizers were inserted into the emission 
and excitation pathway. DOPS LUVs were added to a final concentration of around 0.6 mM. Possible artifacts due to scattering by the LUVs can be minimized by setting the polarizers in an orthogonal arrangement. Fluorescence spectra were corrected for the instrument response after background subtraction and for inner filter effects due to light absorption by acrylamide. All experiments were performed in duplicate.

Data analysis. The emission peak wavelength and width of the emission peak were estimated by fitting the emission spectra $I(\lambda)$ with a log-normal function (11),

for:

$$
\begin{aligned}
& \lambda>\lambda_{\max }-\frac{\rho \Gamma}{\rho^{2}-1}, \\
& I(\lambda)=I_{\max } \exp \left[\frac{\ln 2}{\ln ^{2} \rho} \ln ^{2}\left(1-\frac{\left(\lambda-\lambda_{\max }\right)\left(\rho^{2}-1\right)}{\rho \Gamma}\right)\right]
\end{aligned}
$$

and for:

$$
\lambda<\lambda_{\max }-\frac{\rho \Gamma}{\rho^{2}-1}, I(\lambda)=0
$$

where $\rho$ describes the asymmetry of the peak, $\Gamma$ is the width at half maximum of the spectrum and $\lambda_{\max }$ is the fluorescence intensity at the peak wavelength.

Fluorescence quenching experiments were analyzed by creating Stern-Volmer plots, which plots the quenching ratio as a function of the concentration of quencher present. The fluorescence emission intensity was determined by integrating the emission spectrum from $310 \mathrm{~nm}$ to $450 \mathrm{~nm}$. The quenching curves were fit with the modified form of the Stern-Volmer equation,

$$
\frac{F_{0}}{F}=\left(1+K_{s v}[Q]\right) e^{V[Q]}
$$

where $F_{0}$ is the fluorescence intensity in the absence of quencher, $K_{s v}$ is the Stern-Volmer constant, $[Q]$ is the molar concentration of quencher and $V$ is the static quenching constant.

\section{Acknowledgements}

Some of the $\alpha$-synuclein tryptophan mutants were a kind gift of Asaf Grupi and Elisha Haas, Bar Ilan University, Israel. Most of the acrylamide titrations were performed by Kirsten van Leijenhorst-Groener. 


\section{References}

(1) Kayed, R., Head, E., Thompson, J. L., McIntire, T. M., Milton, S. C., Cotman, C. W., and Glabe, C. G. (2003) Common structure of soluble amyloid oligomers implies common mechanism of pathogenesis. Science 300, 486-489.

(2) Kayed, R., Sokolov, Y., Edmonds, B., McIntire, T. M., Milton, S. C., Hall, J. E., and Glabe, C. G. (2004) Permeabilization of lipid bilayers is a common conformation-dependent activity of soluble amyloid oligomers in protein misfolding diseases. J. Biol. Chem. 279, 46363-46366.

(3) Wood, S. J., Wypych, J., Steavenson, S., Louis, J. C., Citron, M., and Biere, A. L. (1999) alpha-synuclein fibrillogenesis is nucleation-dependent - Implications for the pathogenesis of Parkinson's disease. J. Biol. Chem. 274, 19509-19512.

(4) Volles, M. J., Lee, S. J., Rochet, J. C., Shtilerman, M. D., Ding, T. T., Kessler, J. C., and Lansbury, P. T., Jr. (2001) Vesicle permeabilization by protofibrillar alpha-synuclein: implications for the pathogenesis and treatment of Parkinson's disease. Biochemistry 40, 7812-7819.

(5) Apetri, M. M., Maiti, N. C., Zagorski, M. G., Carey, P. R., and Anderson, V. E. (2006) Secondary structure of alpha-synuclein oligomers: Characterization by Raman and atomic force microscopy. J. Mol. Biol. 355, 63-71.

(6) Danzer, K. M., Haasen, D., Karow, A. R., Moussaud, S., Habeck, M., Giese, A., Kretzschmar, H., Hengerer, B., and Kostka, M. (2007) Different species of alpha-synuclein oligomers induce calcium influx and seeding. J. Neurosci. 27, 9220-9232.

(7) Dusa, A., Kaylor, J., Edridge, S., Bodner, N., Hong, D. P., and Fink, A. L. (2006) Characterization of oligomers during alpha-synuclein aggregation using intrinsic tryptophan fluorescence. Biochemistry 45, 2752-2760.

(8) Lashuel, H. A., Petre, B. M., Wall, J., Simon, M., Nowak, R. J., Walz, T., and Lansbury, P. T., Jr. (2002) Alpha-synuclein, especially the Parkinson's disease-associated mutants, forms porelike annular and tubular protofibrils. J. Mol. Biol. 322, 10891102.

(9) van Rooijen, B. D., Claessens, M., and Subramaniam, V. (2008) Membrane binding of oligomeric alpha-synuclein depends on bilayer charge and packing. FEBS Lett. 582, 3788-3792. 
(10) van Rooijen, B. D., Claessens, M. M. A. E., and Subramaniam, V. (2009) Lipid bilayer disruption by oligomeric alpha-synuclein depends on bilayer charge and accessibility of the hydrophobic core. Biochim. Biophys. Acta-Biomembranes 1788, 1271-1278.

(11) Ladokhin, A. S., Jayasinghe, S., and White, S. H. (2000) How to measure and analyze tryptophan fluorescence in membranes properly, and why bother? Anal. Biochem. 285, 235-245.

(12) Vivian, J. T., and Callis, P. R. (2001) Mechanisms of tryptophan fluorescence shifts in proteins. Biophys. J. 80, 2093-2109.

(13) Eftink, M. R., and Ghiron, C. A. (1981) Fluorescence Quenching Studies With Proteins. Anal. Biochem. 114, 199-227.

(14) Lee, J. C., Lai, B. T., Kozak, J. J., Gray, H. B., and Winkler, J. R. (2007) alpha-Synuclein tertiary contact dynamics. J. Phys. Chem. B 111, 2107-2112.

(15) Winkler, G. R., Harkins, S. B., Lee, J. C., and Gray, H. B. (2006) alpha-synuclein structures probed by 5 -fluorotryptophan fluorescence and F-19 NMR spectroscopy. J. Phys. Chem. B 110, 70587061.

(16) Kaylor, J., Bodner, N., Edridge, S., Yamin, G., Hong, D. P., and Fink, A. L. (2005) Characterization of oligomeric intermediates in alpha-synuclein fibrillation: FRET studies of Y125W/Y133F/Y136F alpha-synuclein. J. Mol. Biol. 353, 357372.

(17) Weinreb, P. H., Zhen, W., Poon, A. W., Conway, K. A., and Lansbury, P. T., Jr. (1996) NACP, a protein implicated in Alzheimer's disease and learning, is natively unfolded. Biochemistry 35, 1370913715 .

(18) Morar, A. S., Olteanu, A., Young, G. B., and Pielak, G. J. (2001) Solvent-induced collapse of alpha-synuclein and acid-denatured cytochrome c. Protein Sci. 10, 2195-2199.

(19) Dedmon, M. M., Lindorff-Larsen, K., Christodoulou, J., Vendruscolo, M., and Dobson, C. M. (2005) Mapping long-range interactions in alpha-synuclein using spin-label NMR and ensemble molecular dynamics simulations. J. Am. Chem. Soc. 127, 476477.

(20) Del Mar, C., Greenbaum, E. A., Mayne, L., Englander, S. W., and Woods, V. L. (2005) Structure and properties of alpha-synuclein and other amyloids determined at the amino acid level. Proc. Natl. Acad. Sci. U. S. A. 102, 15477-15482. 
(21) Giasson, B. I., Murray, I. V. J., Trojanowski, J. Q., and Lee, V. M. Y. (2001) A hydrophobic stretch of 12 amino acid residues in the middle of alpha-synuclein is essential for filament assembly. J. Biol. Chem. 276, 2380-2386.

(22) Der-Sarkissian, A., Jao, C. C., Chen, J., and Langen, R. (2003) Structural organization of alpha-synuclein fibrils studied by sitedirected spin labeling. J. Biol. Chem. 278, 37530-37535.

(23) Miake, H., Mizusawa, H., Iwatsubo, T., and Hasegawa, M. (2002) Biochemical characterization of the core structure of alphasynuclein filaments. J. Biol. Chem. 277, 19213-19219.

(24) Lakowicz, J. R. (1999) Principles of fluorescence spectroscopy, 2nd ed., Kluwer Academic/Plenum, New York.

(25) Eftink, M. R., and Hagaman, K. A. (1985) Fluorescence Quenching Of The Buried Tryptophan Residue Of Cod Parvalbumin. Biophys. Chem. 22, 173-180.

(26) Quist, A., Doudevski, I., Lin, H., Azimova, R., Ng, D., Frangione, B., Kagan, B., Ghiso, J., and Lal, R. (2005) Amyloid ion channels: a common structural link for protein-misfolding disease. Proc. Natl. Acad. Sci. U. S. A. 102, 10427-10432.

(27) Davidson, W. S., Jonas, A., Clayton, D. F., and George, J. M. (1998) Stabilization of alpha-synuclein secondary structure upon binding to synthetic membranes. J. Biol. Chem. 273, 9443-9449.

(28) Eliezer, D., Kutluay, E., Bussell, R., Jr., and Browne, G. (2001) Conformational properties of alpha-synuclein in its free and lipidassociated states. J. Mol. Biol. 307, 1061-1073.

(29) Chandra, S., Chen, X. C., Rizo, J., Jahn, R., and Südhof, T. C. (2003) A broken alpha-helix in folded alpha-synuclein. J. Biol. Chem. 278, 15313-15318. 


\section{Chapter 7 Membrane permeabilization by oligomeric $\alpha$-synuclein: In search of the mechanism}

How the aggregation of the neuronal protein $\alpha$-synuclein contributes to neuronal toxicity in Parkinson's disease has been the subject of intensive research over the past decade. Recently, attention has shifted from the amyloid fibrils to soluble oligomeric intermediates in the $\alpha$-synuclein aggregation process. It has been hypothesized that these so called oligomers are cytotoxic through the disruption and permeabilization of cellular membranes possibly by forming pore-like complexes in the bilayer. Although the subject of $\alpha$-synuclein oligomer-membrane interaction has attracted much attention, the oligomer pore hypothesis only relies on a small body of evidence. In this chapter we have tried to gain insight into the mechanism of lipid bilayer disruption by $\alpha$-synuclein oligomers using a number of in vitro bilayer systems and assays. Dye efflux from vesicles induced by oligomeric $\alpha \mathrm{S}$ is a fast all-or-none process. A newly developed assay based on a dextran coupled dye showed that nonequilibrium processes dominate the disruption of the vesicles. The membrane permeability appears to be caused by large defects in the bilayer as even a 70 $\mathrm{kDa}$ dextran could escape the vesicle interior. The bilayer defects may be due to the instability of lipid bilayers with a high negative charge density. Vesicles from negatively charged lipids were generally unstable to protein adsorption. The presence of calcium stabilizes the vesicles. Under these conditions the properties of the efflux process might be consistent with a pore model. Permeabilization was selective to the molecular weight of the marker and persisted after equilibration of the oligomer containing membrane. However, in the presence of calcium, non-equilibrium processes at the initial stages after oligomer addition have a major contribution to the permeability of the membrane, which points to the occurrence of bilayer defects. 


\section{Introduction}

In recent years attention has shifted to the early stages of the aggregation process of $\alpha \mathrm{S}$. Oligomeric intermediates in the aggregation of $\alpha \mathrm{S}$ have been found to be more toxic to cells than monomeric or fibrillar forms of the protein $(1,2)$. A possible mechanism by which oligomers could be toxic is through the disruption and permeabilization of cellular membranes (3-5). To test this hypothesis mainly in vitro model systems have been used. Oligomeric $\alpha \mathrm{S}$ has been shown to permeabilize synthetic phospholipid vesicles $(5,6)$. The solute efflux from these vesicles was found to be selective for the molecular weight of the entrapped marker and might thus occur through a pore-like mechanism (7). Formation of pore-like complexes by $\alpha \mathrm{S}$ is supported by the observation of donut shaped protein particles by electron microscopy and $\operatorname{AFM}(8,9)$. In addition, single channel activities have been observed in planar lipid bilayer systems (8). However, the literature data are not conclusive. A thorough electrophysiological characterization of the hypothesized $\alpha \mathrm{S}$ pore is still lacking. This is critical, as there are many ways in which proteins can destabilize the membrane integrity (10). Whether oligomer membrane disruption indeed has a role as an important cytotoxic mechanism is still unclear. Even in vitro, the process of oligomer membrane disruption is still not well understood.

In this chapter we have tried to determine the mechanism by which oligomeric $\alpha \mathrm{S}$ induces membrane permeabilization. Results from dynamic light scattering (DLS), confocal microscopy and a fluorescence re-quenching assay indicate that disruption of vesicles occurs in a rapid all-or-none fashion in which the overall vesicle morphology is not changed. However, the disruption of the membrane most likely occurs through the appearance of large defects in the bilayer, since high molecular weight dextran molecules could escape the vesicle interior. This disruption mechanism could be caused by the instability of the negatively charged membranes. The vesicles composed of negatively charged lipids proved unstable to the adsorption of different unrelated proteins. However, in the presence of calcium, oligomers possibly permeabilize the membrane through a pore-like process. A newly developed method based on a dextran coupled dye and an external quencher, allows us to measure the permeability of the membrane as a function of equilibration time after the addition of oligomeric $\alpha \mathrm{S}$. The results from this assay show that even in the presence of calcium, non-equilibrium phenomena have a large contribution in the disruption process. However, membrane permeabilization was sensitive to the size of the marker and permeabilization was not transient and persisted even after equilibration of the membrane. 


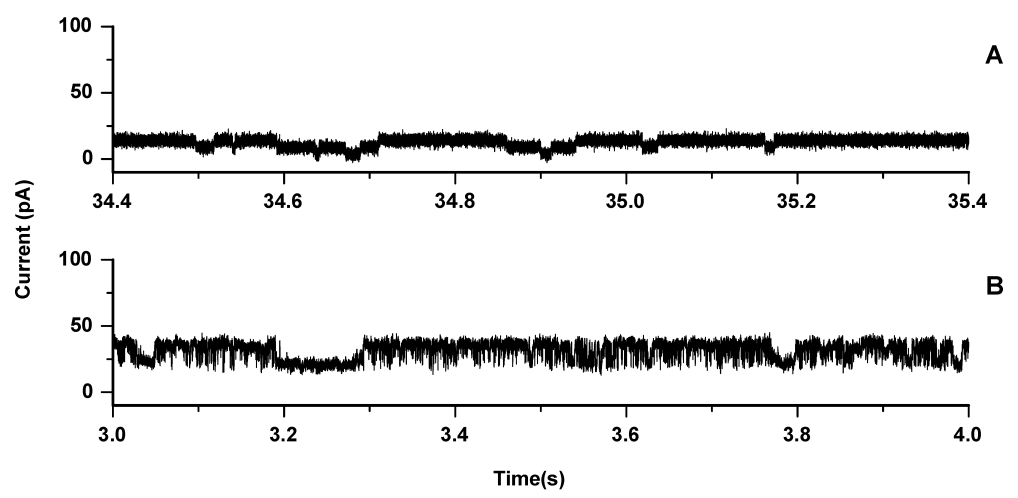

Figure 1. Single channel recordings Recordings of channel events in a POPG planar lipid bilayer. The current was recorded with a transmembrane potential of $100 \mathrm{mV}$ in the presence of $500 \mathrm{nM}$ oligomeric $\alpha \mathrm{S}$. Such activity was only observed if the membrane was formed and broken repeatedly. The top and bottom panels show the events observed in two separate experiments.

\section{Results}

It has been suggested that membrane permeabilization by oligomeric $\alpha \mathrm{S}$ occurs through the insertion of pore-like complexes in the membrane. The planar lipid bilayer technique is well suited to characterize proteins that form ion permeable pores in lipid bilayers and has been used to show pore-like properties of $\alpha \mathrm{S}$ oligomers (8). However, the technique is extremely sensitive and prone to artifacts, as the observed activities are essentially single molecule events. To prove a pore-like model, a thorough, reproducible study is necessary.

We and others, have demonstrated that LUVs of POPG are vulnerable to permeabilization by oligomeric $\alpha S(7,11)$. Therefore, POPG was used to create planar lipid bilayers. The monolayer folding technique was used to create solvent free lipid bilayers (12). The resulting POPG bilayers were only stable for a short time, generally a few minutes to less then a minute (at a membrane voltage of $100 \mathrm{mV}$ ), thereby making it very difficult to assess the effect of the protein on membrane stability. After a stable bilayer was obtained a small amount of oligomeric protein (final concentration $200 \mathrm{nM}$ ) was added to the solution on both the cis- and trans-side of the membrane and the solution was thoroughly mixed. No current jumps characteristic of insertion of pore-like proteins were observed under normal conditions. Only if the membrane was broken and reformed repeatedly, by lowering and raising the water-air interface, while mixing intermittently, events which could represent 


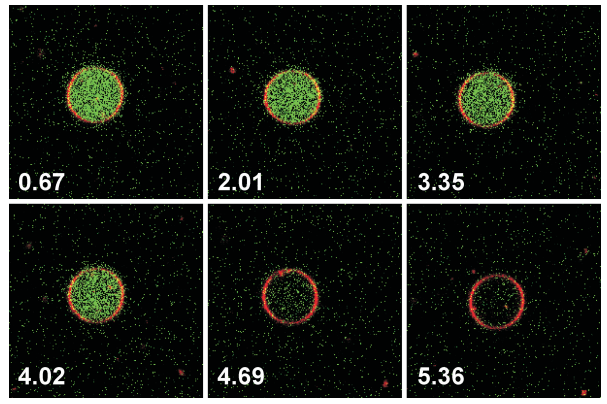

Figure 2. Efflux kinetics Confocal microscopy images of POPG GUVs filled with the dye HPTS (green). The quencher DPX was present on the outside of the vesicles. The GUV membrane was stained with DOPE-Rhodamine (red). The consecutive images (time stamp in seconds) show the kinetics of dye efflux from a single GUV.

pore insertion were sometimes observed (Figure 1). However, these events were very difficult to reproduce, partly because the conditions required for these events also induce membrane instability. The insertion events were analyzed in more depth using the QuB software suite (13). Comparing the results between separate experiments did not reveal a single conductance level or characteristic kinetics. For instance the events shown in Figure 1A showed a conductance level of around $0.05 \mathrm{nS}$ with a long average lifetime of around $129 \mathrm{~ms}$. The experiment shown in Figure 1B revealed conductance levels of $0.14 \mathrm{nS}$ and 0.08 $\mathrm{nS}$ with fast kinetics with a lifetime of around $1.0 \mathrm{~ms}$. Good statistics and reproducibility are necessary for reliable conclusions. Therefore, these results are not sufficient to prove a pore-like mechanism of disruption. We have for instance observed similar current jumps in POPG bilayers in the absence of protein, due to hydrostatic pressure differences between the cis- and transchambers.

To gain more insight into the mechanism of the disruption process, confocal fluorescence microscopy was used to observe the oligomer induced membrane permeability of giant unilamellar vesicles (GUVs). POPG GUVs encapsulating the dye HPTS were created and the lipid dye DOPE-Rhod was added to the lipid composition to visualize the bilayer. The GUVs were diluted from salt free stock solutions into salt containing buffer solutions. To further reduce the signal from un-encapsulated HPTS, the quencher DPX was added to the solution. The resulting GUV solutions were stable and showed good encapsulation of HPTS. When oligomeric $\alpha \mathrm{S}$ was added to the imaging chamber, the fluorescence from the vesicle interior was lost, either through HPTS efflux or DPX influx. By continuously imaging the GUVs just after oligomer 


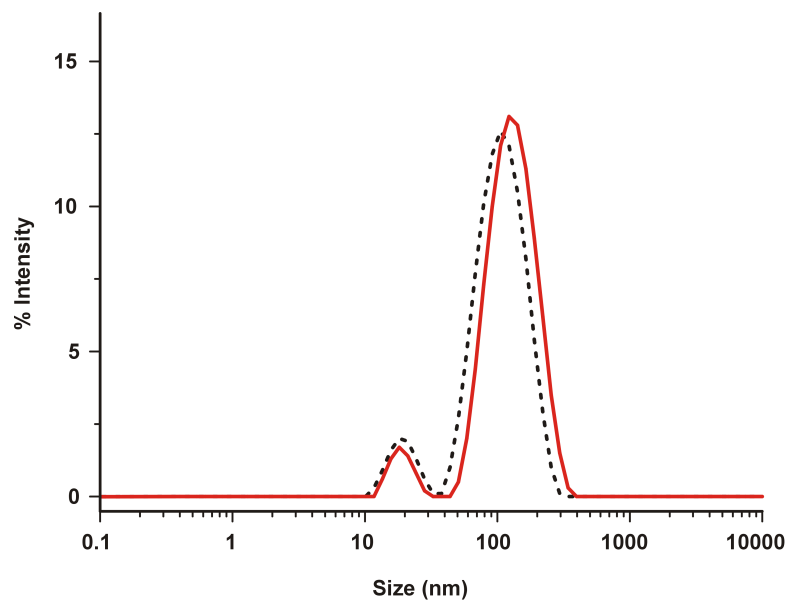

Figure 3. Vesicle integrity DLS on POPG LUVs at a lipid concentration of $20 \mu \mathrm{M}$ in the presence of $1 \mu \mathrm{M}$ oligomeric $\alpha \mathrm{S}$. Size distributions were acquired at different time points after oligomer addition: $t=0$ (black dotted line), $t=15$ minutes (red solid line).

addition, the leakage process could be followed over time. Figure 2 shows several consecutive images after the addition of oligomeric $\alpha \mathrm{S}$. The images show that the leakage process is very fast and that vesicles appear morphologically unchanged.

To confirm that vesicles remain largely intact upon oligomer addition, DLS experiments were performed. $\alpha$ S oligomers were added to POPG LUVs and the particle size distribution was followed over time. Figure 3 shows the characteristic size distribution of LUVs in the presence of oligomeric $\alpha$ S. Just after the addition of oligomers $(t=0)$, two distinct peaks corresponding to a size of around $18 \mathrm{~nm}$ for oligomeric $\alpha \mathrm{S}$ and $110 \mathrm{~nm}$ for the POPG LUVs were observed. After 30 minutes both peaks were still present in the size distribution. The vesicle peak was shifted to slightly larger sizes of around $120 \mathrm{~nm}$. The amount of light scattering from particles increases with increasing size of the particles. Corresponding to the shift in the particle size distribution, the photon count rate of the scattered light increased during the incubation time. The increase in size could be caused by an adsorbed protein layer on the outside of the vesicles, or an increase in surface area of the membrane surface due to protein insertion. Clearly, the LUVs are not completely destroyed or solubilized by oligomeric $\alpha \mathrm{S}$, but remain largely intact over the course of the experiment. 


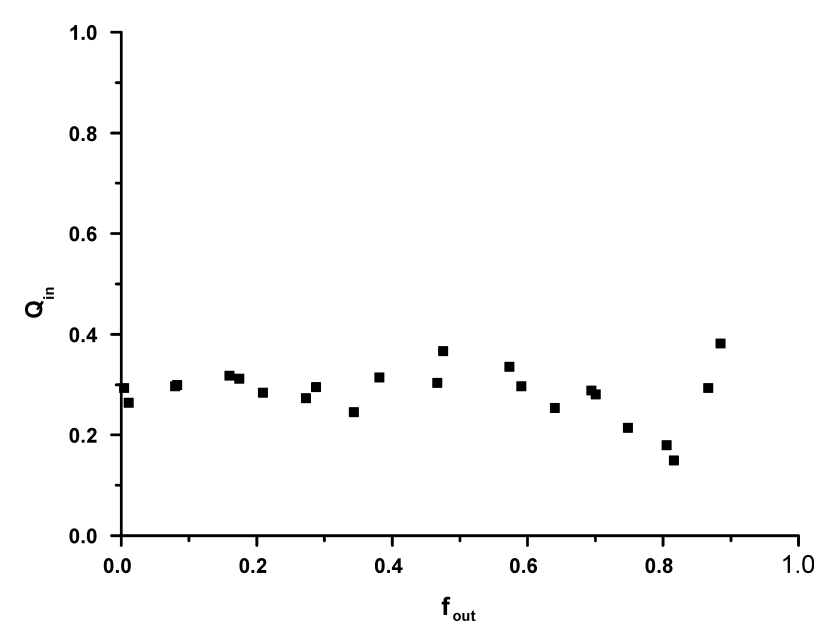

Figure 4. All-or-none release POPG LUVs encapsulating HPTS/DPX were mixed with different amounts of oligomeric $\alpha \mathrm{S}$ to a final lipid concentration of 25 $\mu \mathrm{M}$. The quenching ratio $Q_{i n}$ in the vesicle interior does not change as a function of the amount of escaped dye $f_{\text {out }}$, which indicates that dye efflux from the LUVs occurs through an all-or-none mechanism.

The mechanism by which LUVs release their content upon interaction with oligomeric $\alpha \mathrm{S}$ was further studied by using a re-quenching assay based on the HPTS/DPX dye quencher pair. Dye release can occur either though an all-ornone or a graded mechanism (14). In other words: if half of the encapsulated solute has escaped, this can either be the result of half of the vesicles releasing all their content, or all vesicles releasing half of their content. The dye quencher pair HPTS/DPX was co-encapsulated inside LUVs which causes the fluorescence of HPTS to be quenched by a certain ratio $Q_{i n}$. In the case of allor-none release, the ratio $Q_{i n}$ is independent of the fraction of escaped solute $f_{\text {out }}$. In the case of graded release, assuming there is no preferential release of HPTS over DPX, the ratio $Q_{i n}$, depends on the fraction of escaped solute, since this causes the DPX concentration to drop. The quenching ratio inside the vesicles as a function of the amount of escaped solute is experimentally accessible by titrating DPX to LUVs after solute efflux has occurred, as is described in the Materials and Methods section. In the experiment, POPG LUVs were incubated with different concentrations of oligomeric $\alpha \mathrm{S}$, after which $Q_{\text {in }}$ and $f_{\text {out }}$ were subsequently determined. The results in Figure 4 show that $Q_{\text {in }}$ remains roughly constant with the increase of the escaped fraction of dye $f_{\text {out }}$, indicating that leakage occurs through an all-or-none mechanism. This result 


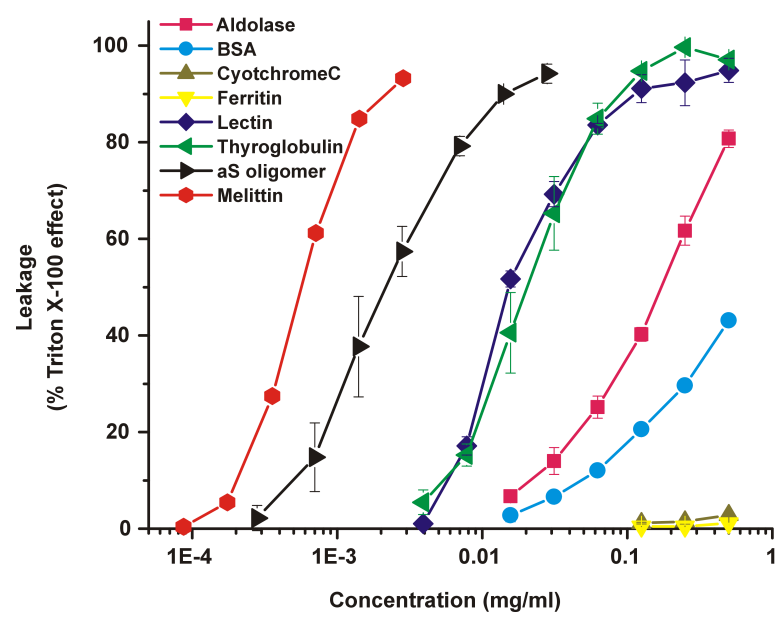

Figure 5. Vesicle stability Stability of POPG LUVs to protein absorption was measured by the calcein efflux induced by the addition of different concentrations of several proteins at a phospholipid concentration of $20 \mu \mathrm{M}$. The error bars indicate the standard deviation $(n=3)$.

supports the data from the confocal microscopy experiment in which dye efflux from GUVs was extremely rapid and proceeded until the vesicle fluorescence intensity inside and outside the GUV were similar.

Since calcein efflux has also been observed upon addition of monomeric and fibrillar $\alpha \mathrm{S}$ (15), disruption of the vesicles might represent a measure of intrinsic vesicle instability rather than a specific mechanism. Therefore, the stability of POPG LUVs to protein adsorption was investigated by adding a number of unrelated proteins. Proteins were selected to have a range of different properties such as molecular mass, charge and biological function. The results are summarized in Figure 5. Bovine cytochrome c (12.3 kDa, pI 9.3) and horse apoferritin (443 kDa, pI 4.3) did not significantly affect vesicle stability. Rabbit aldolase (168 kDa, pI 8.1) and bovine serum albumin (66 kDa, pI 4.9) were able to disrupt $50 \%$ of the POPG LUVs at high concentrations of around 0.2 and $0.5 \mathrm{mg} / \mathrm{ml}$ respectively. Bovine thyroglobulin (660 $\mathrm{kDa}, \mathrm{pI} 4.5)$ and lectin from Lens Culinaris (24 kDa, pI 8.5) were even more effective in inducing calcein efflux from the LUVs with $50 \%$ efflux at approximately $0.02 \mathrm{mg} / \mathrm{ml}$. As a positive control, the membrane disrupting peptide melittin from honey bee venom, induced a $50 \%$ leakage at around $0.0005 \mathrm{mg} / \mathrm{ml}$. Oligomeric $\alpha \mathrm{S}$ induced $50 \%$ leakage at $\sim 0.0018 \mathrm{mg} / \mathrm{ml}$. No correlation between the induced leakage and the protein size or charge was observed, so the disruptive effect 

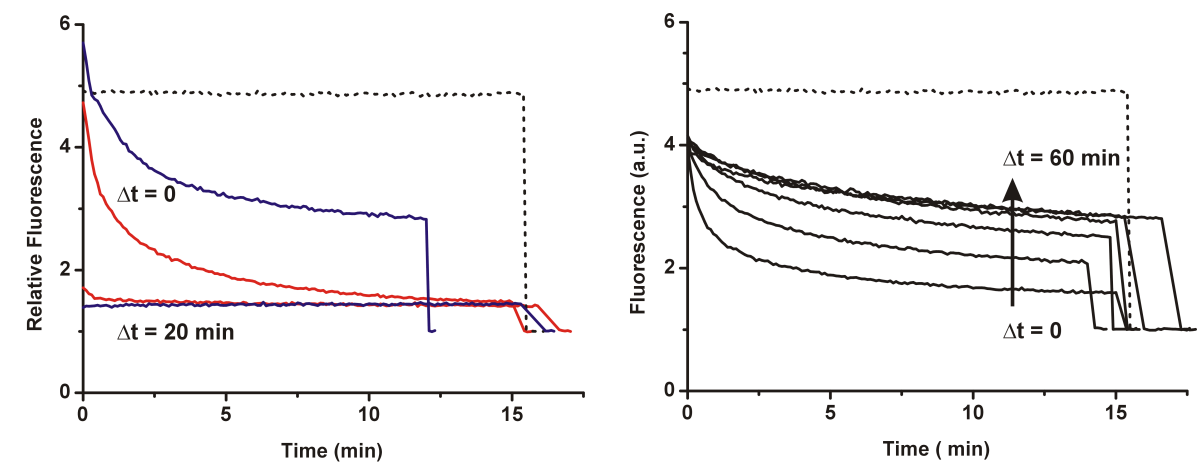

Figure 6. Transient permeabilization To measure if vesicle permeabilization was transient POPG LUVs encapsulating dextran-PTS were prepared. The quencher DPX was added $\Delta t$ after mixing the vesicles with oligomeric $\alpha \mathrm{S}$ to a final concentration of $0.25 \mathrm{mM}$ lipid and $1.5 \mu \mathrm{M} \alpha \mathrm{S}$. The dotted line is a background curve in the absence of protein. After $\sim 15$ minutes Triton X-100 is added to disrupt all vesicles, the curves are normalized to this final value. (A) In the absence of calcium the dextran-PTS conjugate escapes the vesicle interior upon protein interaction $(3 \mathrm{kDa}$ red lines, $70 \mathrm{kDa}$ blue lines). (B) POPG LUVs encapsulating a $3 \mathrm{kDa}$ dextran-PTS. In the presence of $5 \mathrm{mM} \mathrm{Ca}^{2+}$, the $3 \mathrm{kDa}$ dextran-PTS remains in the vesicle interior. The amount of DPX influx is reduced upon increasing equilibration time $(\Delta t=0$, $0.5,1,5,20$ and 60 minutes).

is determined by specific structural features rather than broad biochemical characteristics. Oligomeric $\alpha \mathrm{S}$ was more effective in disrupting POPG LUVs than many of the other proteins used, and was around 4 times less effective than melittin which is a well-characterized membrane disrupting peptide. Although disruption of LUVs is thus most likely related to the structural properties of the $\alpha \mathrm{S}$ oligomer, vesicle instability to protein absorption clearly is an issue for negatively charged LUVs.

The results that negatively charged LUVs are unstable to protein adsorption, that vesicle efflux from single GUVs is rapid, and that leakage occurs through an all-or-none mechanism, led us to hypothesize that the dye efflux could be due to a transient non-equilibrium process. Membrane defects could occur upon binding of the oligomers to the membrane. For instance, the bilayer might be perturbed by the increased surface area in the outer leaflet upon protein adsorption. After a certain period the bilayer has accommodated the protein and could reach a new equilibrium state in which no defects are present. To be able to assess the permeability of the membrane at a certain time point after the addition of oligomeric $\alpha \mathrm{S}$, a new assay was developed. An 
isothiocyanate derivative of HPTS was conjugated to amine-modified dextran of different molecular weights $(3 \mathrm{kDa}, 10 \mathrm{kDa}$ and $70 \mathrm{kDa})$. If the membrane defects are small, the dextran-PTS conjugate will stay encapsulated in the LUV interior. Membrane permeability can subsequently be measured by adding DPX, which quenches the dextran-PTS fluorescence if it can cross the vesicle membrane.

In the experiment POPG LUVs containing the dextran-PTS conjugate were mixed with oligomeric $\alpha \mathrm{S}$. DPX was either present from the start of the experiment or added after a certain reaction time. Figure $6 \mathrm{~A}$ shows how the dextran-PTS fluorescence changes over time. If DPX is present from the start of the experiments the fluorescence is rapidly quenched. However, if the LUVs were incubated with oligomeric $\alpha \mathrm{S}$ for 20 minutes, the fluorescence was immediately lost on DPX addition. This indicates that the dextran conjugate most likely has escaped from the vesicle interior. This was also the case for the 70 $\mathrm{kDa}$ dextran conjugate indicating that the initial membrane defects are large.

It has been reported that solute efflux induced by oligomeric $\alpha \mathrm{S}$ is size selective in the presence of calcium ions (7). Figure $6 \mathrm{~B}$ shows the results for the time dependent membrane permeability in the presence of calcium. When oligomeric $\alpha \mathrm{S}$, LUVs and DPX were mixed at the same time a clear decrease in fluorescence intensity was observed. Subsequently, if the LUVs were preincubated with oligomeric $\alpha \mathrm{S}$ a certain time before DPX addition, the majority of dextran-dye conjugate was retained in the vesicle interior. This allowed us to map the permeability of the membrane to DPX as a function of incubation time of the LUV-oligomer mixture. The results indicate that the membrane permeability is largest immediately after addition of the $\alpha \mathrm{S}$ oligomers. The initial defects seem to heal swiftly and permeability goes down within 5 minutes to a stable level. However, this level of permeability was not reduced any further even after 60 minutes of equilibration time of LUVs. This implies that in the presence of calcium the vesicle permeabilization is not a transient process. However, non-equilibrium phenomena in the first minute after oligomer addition to LUVs have a major contribution on the observed membrane permeability.

\section{Discussion}

Oligomeric intermediates in the aggregation process of $\alpha \mathrm{S}$ are often considered as possible toxic species. The disruption and permeabilization of cellular membranes has been suggested as a likely mechanism of toxicity $(2,7)$. However, this hypothesis is only supported by a small body of experimental evidence and the molecular details of the process are not known. In this chapter we have tried to determine the mechanisms through which oligomeric $\alpha \mathrm{S}$ disrupts and 
permeabilizes the lipid bilayer.

A possible mechanism by which oligomeric $\alpha \mathrm{S}$ can cause membrane permeabilization is through the formation of pore-like structures in the membrane. Single channel recording is an excellent technique to characterize pore insertion events. However, for oligomeric $\alpha \mathrm{S}$ the technique has yielded varying results (3, 8 ). Both single channel-like events as well as an increase in permeability without distinct channel events have been observed. A disadvantage of the planar bilayer technique is that it is extremely sensitive and one has to ensure the data is reproducible. Although we have sometimes observed pore-like events, these events were not easily reproduced. The instability of planar bilayers composed of negatively charged lipids, even in the absence of oligomers, posed major problems. We have not seen evidence for the increased permeability without channel events (3), which is thought to be caused by membrane thinning or a change of the membrane dielectric structure (16). However recent evidence indicates that such events may be caused by the presence of residual amounts of solvents that are used in some protocols of oligomer preparation (17). The results from the planar lipid bilayer technique thus seem inconclusive. Experimental conditions that optimize the membrane stability and improve the protein insertion need to be identified.

To overcome these difficulties and to identify mechanistic aspects of the permeabilization process, several assays based on LUV and GUV systems were used. Confocal microscopy on GUVs shows that the disruption process is extremely fast and without changes to vesicle morphology. Results from DLS experiments confirm that LUVs stay largely intact upon oligomer interaction. Additionally, LUVs containing the dye quencher pair HPTS/DPX were used to show that vesicle disruption occurs through an all-or-none mechanism. These data led to the hypothesis that vesicle permeabilization might be a transient event. In such a process defects occur upon protein adsorption, but are healed when the membrane reaches a new equilibrium state. In order to measure transient permeabilization, an assay was developed based on the quencher DPX and a derivative of the dye HPTS conjugated to high molecular weight dextrans. This assay allows measuring the membrane permeability at different equilibration times after addition of the protein. In the absence of calcium however membrane defects were too large, and even the $70 \mathrm{kDa}$ dextran was able to escape the LUV interior. It is unlikely that the inner radius of the hypothesized $\alpha \mathrm{S}$ pore is large enough to allow diffusion of the $70 \mathrm{kdA}$ dextran (7-9). Thus in the absence of calcium, disruption of POPG vesicles most likely does not occur through a pore-like mechanism but rather through large defects.

These defects could be caused by the instability of the negatively charged LUVs. Many different unrelated proteins were found to cause calcein efflux from POPG LUVs if added in sufficient amounts. It has indeed been reported 
that vesicles containing high amounts of negatively charged vesicles are ruptured more easily by mechanical forces (18). However, the disruption of the vesicles is not merely related to the vesicle instability but also to the structure of oligomeric $\alpha \mathrm{S}$. Compared to the different proteins added to the vesicles, disruption by oligomeric $\alpha \mathrm{S}$ occurred in a concentration range more similar to melittin, which is a well known membrane disruptive protein. The finding that other proteins were less efficient in permeabilizing the membrane could be due to a difference in binding affinity for the POPG LUVs. However, the mode of binding is clearly important. It was reported in Chapters 3 and 5 that monomeric $\alpha \mathrm{S}$ has a higher lipid affinity but does not disrupt the vesicles as efficiently. Under specific conditions monomeric $\alpha \mathrm{S}$ can even stabilize the membrane. Therefore, the oligomers must contain structural properties that make it highly destabilizing to the bilayer integrity.

We have previously chosen to characterize the process of membrane permeabilization by oligomeric $\alpha \mathrm{S}$ in the absence of calcium to reduce the complexity of the system. The selectivity of solute efflux as known from literature was measured in the presence of calcium (7). The findings presented in this chapter are in good agreement with this previous report. In the presence of calcium a size selectivity for marker diffusion across the POPG membrane was observed. The addition of oligomeric $\alpha \mathrm{S}$ to LUVs did not induce the release of $3 \mathrm{kDa}$ dextran-PTS and the quencher DPX (422 Da) was able to cross the membrane and quench the encapsulated fluorescent dextran conjugate. However, care should be taken in considering these findings as direct evidence for the pore model. Non-equilibrium processes still contribute to the disruption process, which points to the presence of membrane defects. Membrane permeability is greatest immediately after the addition of oligomeric $\alpha \mathrm{S}$. The permeability of the membrane is not transient but goes down to a plateau level after equilibration. For a pore-like mechanism one would expect that the initial permeability is lowest and subsequently goes up to a plateau level, since the protein first has to bind and insert into the bilayer. An alternative explanation to the pore model is that in the presence of calcium, leakage still occurs through defects due to bilayer instability. The average size and lifetime of the defects might be related to the degree of membrane instability and membrane stress. In fact, even protein free lipid bilayers are known to contain unstable defects with finite sizes under conditions of stress (19). Thus, such a mechanism might be possible and would also explain our observations of single channel-like events with inconsistent conductance levels.

Summarizing, $\alpha \mathrm{S}$ oligomers are clearly a membrane active species, and have a destabilizing effect on the bilayer integrity. Oligomers are relatively efficient at disrupting the vesicles compared to other proteins, which may be linked to structural features of the oligomeric complex. A pore-like mechanism is possi- 
ble, although non-equilibrium processes very likely have a major contribution to the observed permeabilization. Defects due to protein adsorption and vesicle instability do occur. Therefore extreme care should be used when using highly negatively charged vesicles as a model system for detecting oligomer membrane permeabilization.

\section{Materials and Methods}

Purification of monomeric and oligomeric $\alpha S$. Purification of $\alpha \mathrm{S}$ and preparation of oligomeric species was performed as in Chapter 2.

Planar lipid bilayer measurements. Solvent free planar lipid membranes were formed by the joining of lipid monolayers according to the method of Montal and Mueller (12). A small aperture (40-70 $\mu \mathrm{m})$ was punched through a $40 \mu \mathrm{m}$ thick Teflon film by an electric discharge. The film was clamped between two Teflon cuvettes and the aperture was pretreated on both sides with $3 \mu \mathrm{l} 1 \%(\mathrm{v} / \mathrm{v})$ hexadecane in hexane. After evaporation of the hexane, $250 \mu \mathrm{l}$ of $10 \mathrm{mM}$ HEPES $\mathrm{pH} 7.4,150 \mathrm{mM} \mathrm{KCl}$ was added to both sides of the film. Lipid monolayers were formed on top of the solution by adding $1 \mu \mathrm{l}$ of lipids in pentane $(5 \mathrm{mg} / \mathrm{ml})$. The solvent was allowed to evaporate for 10 min. Lipid bilayers spontaneously formed by lowering and raising the liquid level on one side of the aperture. Membrane conductance was measured by two $\mathrm{Ag} / \mathrm{AgCl}$ electrodes on the cis- and trans-side of the membrane. The electrodes were connected to an Axopatch 200B amplifier (Axon instruments). Signals were digitized using a Digidata 1440A (Axon instruments) digitizer and stored on a personal computer for further analysis. The cuvette and amplifier head stage were located in a Faraday cage inside an acoustically isolated room to minimize environmental noise contributions. Membrane quality was assessed by measuring bilayer capacitance and conductance. The data was analyzed using the QUB software package (13).

Preparation of GUVs. Around $0.05 \%$ DOPE-Rhod was added to the membrane lipid composition. Approximately $1 \mathrm{mg}$ of total lipid in chloroform was deposited in a glass vial and dried using nitrogen gas. Residual chloroform was removed by drying under vacuum for 4 hours. GUVs were subsequently created by gentle hydration. The lipid film was hydrated for 6 hours at room temperature in $500 \mu \mathrm{l}$ of sucrose solution containing $20 \mu \mathrm{M}$ of HPTS of equal osmolarity to $10 \mathrm{mM}$ HEPES pH 7.4, $150 \mathrm{mM} \mathrm{NaCl}$.

Confocal Microscopy. Confocal microscopy was performed on a Zeiss LSM 510 confocal microscope. DOPE-Rhod was excited at $543 \mathrm{~nm}$ using a green HeNe laser and HPTS was excited using the $488 \mathrm{~nm}$ Argon laser line. Emission from both dyes was measured simultaneous using the appropriate dichroic mirrors and filter sets. HPTS filled GUVs were diluted to approximately 30 
The mechanism of membrane permeabilization

$\mu \mathrm{M}$ lipid concentration in $10 \mathrm{mM}$ HEPES, $\mathrm{pH}$ 7.4, $150 \mathrm{mM} \mathrm{NaCl}$ and $10 \mathrm{mM}$ DPX. A solution of oligomeric $5 \mu \mathrm{M} \alpha \mathrm{S}$ in $10 \mathrm{mM}$ HEPES pH 7.4 and $150 \mathrm{mM}$ $\mathrm{NaCl}$ was injected at one end of the imaging chamber and allowed to slowly diffuse though the chamber (final concentration $\sim 1.5 \mu \mathrm{M}$ ). GUVs were followed by confocal microscopy in continuous imaging mode. Imaging conditions were optimized with respect to the temporal resolution.

Preparation of LUVs. POPG was obtained from Avanti Polar lipids and used without further purification. LUVs were prepared by extrusion. First a thin lipid film was formed by drying around $0.5 \mathrm{mg}$ of lipid in a glass tube using a gentle stream of N2. Trace amounts of solvent were removed by drying under vacuum for at least 4 hours. The lipid film was then hydrated by adding a solution, $150 \mathrm{mM} \mathrm{NaCl}, 10 \mathrm{mM}$ HEPES, pH 7.4 containing the solute to be encapsulated in the vesicle, unless noted otherwise. Hydration was continued for 1 hour, with vortexing approximately every 15 minutes. The sample was subsequently subjected to 5 freeze-thaw cycles by dipping into liquid nitrogen and thawing above the lipid phase transition temperature. The resulting solution was extruded 11 times through a polycarbonate membrane filter with a $100 \mathrm{~nm}$ pore size. This procedure was repeated once with a new filter. Unencapsulated dye was separated from the vesicles by gel filtration through a PD10 column packed with Sephadex G-100 (GE) unless noted otherwise. The total phospholipid concentration was determined according to the protocol of Chen et al. (20).

Calcein Efflux assay. The calcein efflux assay was performed as described in Chapter 3.

$D L S$. The DLS experiments were performed on a Malvern Zetasizer Nano zs. Oligomeric $\alpha \mathrm{S}$ and POPG LUVs in $10 \mathrm{mM}$ Hepes pH 7.4 and $150 \mathrm{mM} \mathrm{NaCl}$ were mixed to a final concentration of $20 \mu \mathrm{M}$ lipid and $1 \mu \mathrm{M} \alpha \mathrm{S}$ oligomers and placed in a $3 \mathrm{~mm}$ path length quartz cuvette. Light scattering was measured over 15 minutes in 5 second runs. Data analysis was performed using the Malvern DTS software.

Fluorescence re-quenching assay. The assay was performed similar to the protocol as published by Ladokhin et al. (14), with the modification that the dye 8-Hydroxypyrene-1,3,6-trisulfonic acid (HPTS) was used in combination with the quencher p-Xylene-bis(N-pyridinium bromide) (DPX). Both compounds were obtained from Sigma-Aldrich. The assay tries to discern two different scenarios: If $50 \%$ content release from vesicles is observed is this caused by half of the vesicles releasing all their content (all-or-none mechanism) or by all vesicles releasing half of their content (graded release).

The quenching ratio of HPTS is dependent on the DPX concentration. If vesicles are disrupted by an all-or-none mechanism, the quenching ratio inside the vesicles that are still intact has not changed. For graded release the 
quenching ratio inside the vesicles changes due to the efflux of DPX (assuming that DPX and HPTS have a similar probability to be released from the vesicle). Thus by estimating the quenching ratio of the vesicle interior $\left(Q_{i n}\right)$ as a function of vesicle disruption represented by the fraction of released dye $f_{\text {out }}$, it is possible to discern graded $\left(Q_{\text {in }}\right.$ changes with $\left.f_{\text {out }}\right)$ from all-or-none release $\left(Q_{\text {in }}\right.$ remains constant with respect to $\left.f_{\text {out }}\right)$. Both $Q_{\text {in }}$ and $f_{\text {out }}$ are experimentally accessible through the following equation:

$$
Q_{\text {tot }}=Q_{\text {out }} f_{\text {out }}+Q_{\text {in }}\left(1-f_{\text {out }}\right)
$$

In a typical experiment the protein is allowed to interact with vesicles causes a fraction of dye efflux or $f_{\text {out }}$ and a certain value of $Q_{i n}$. After leakage from the vesicles has reached a plateau value, DPX is added in aliquots, which leads to several values of $Q_{\text {out }}$ that are determined from a calibration curve. From measuring the fluorescence intensity after each addition of DPX, $Q_{t o t}$ can be calculated, since $Q_{t o t}=F_{\max } / F$. The maximum possible value of fluorescence $\left(F_{\max }\right)$ is determined by breaking all vesicles through the addition of triton X-100 after the final DPX addition. By plotting $Q_{t o t}$ as a function of $Q_{\text {out }}$, the values for $f_{\text {out }}$ and $Q_{\text {in }}$ are determined from the slope and intercept of a linear fit.

POPG LUVs filled with 1mM HPTS, $2.5 \mathrm{mM}$ DPX in $10 \mathrm{mM}$ HEPES, pH 7.4 and $150 \mathrm{mM} \mathrm{NaCl}$ were prepared by extrusion. HPTS fluorescence was measured on a Varian Eclipse fluorimeter, with the excitation wavelength at 440 $\mathrm{nm}$ (10 nm slit) and emission was measured from $480 \mathrm{~nm}$ to $530 \mathrm{~nm}$ (10 nm slit). Vesicles were mixed with $\alpha \mathrm{S}$ oligomers at several protein concentrations and a final lipid concentration of $25 \mu \mathrm{M}$. After 30 minutes the HPTS fluorescence was measured and subsequently DPX was added in aliquots to a final concentration of $3 \mathrm{mM}$. After each addition the HPTS emission spectrum was measured. After the final DPX addition $0.5 \%$ (weight/volume) Triton X-100 was added and a spectrum was measured.

Transient permeabilization assay. For the transient permeabilization assay, amine modified dextran molecules were labeled with an isothiocynate derivative of the dye HPTS. The resulting dextran-PTS conjugate could be effectively quenched with DPX. Dextran (3 kDa, $10 \mathrm{kDa}$ and $70 \mathrm{kDa})$ functionalized with amino groups was obtained from Invitrogen. The dye 8-Isothiocyanatopyrene1,3,6-trisulfonic acid trisodium salt was obtained from Sigma-Aldrich. For dextran labeling around $2.5 \mathrm{mg}$ of dye and $7.5 \mathrm{mg}$ dextran were dissolved in 400 $\mu \mathrm{l}$ sodium bicarbonate buffer $\mathrm{pH}$ 9. After a two hour incubation, free dye was separated from the dextran bound dye by size exclusion chromatography. For the $3 \mathrm{kDa}$ and $10 \mathrm{kDa}$ dextran a Superose 12 column (GE) was used. The $70 \mathrm{kDa}$ dextran was separated on a Superdex 200 column (GE), with $10 \mathrm{mM}$ HEPES pH 7.4 and $50 \mathrm{mM} \mathrm{NaCl}$ as elution buffer. The fractions containing 
dye labeled dextran were pooled and concentrated using a Vivaspin (Sartorius) centrifugal concentrator with the appropriate molecular weight cutoff. For hydration around $150 \mu \mathrm{l}$ of the resulting labeled dextran solution was added to 2 $\mathrm{mg}$ of dried POPG lipids. After extrusion the LUVs were separated from the free dextran by size exclusion chromatography on a Superdex 200 column. Fluorescence from the dextran-PTS filled LUVs was measured on a Varian Eclipse fluorimeter. The excitation wavelength was $430 \mathrm{~nm}$ with a $5 \mathrm{~nm}$ slit width and emission was measured at $500 \mathrm{~nm}$ with a $20 \mathrm{~nm}$ slit width. In a typical experiment the dextran-PTS emission was followed over time at $t=0,50 \mu \mathrm{l}$ of LUVs were added to $50 \mu \mathrm{l}$ protein solution, to a final concentration of $250 \mu \mathrm{M}$ phospholipids and $1.5 \mu \mathrm{M} \alpha \mathrm{S}$ oligomers. After varying reaction times $5 \mu \mathrm{l}$ of $100 \mathrm{mM}$ DPX was added.

\section{Acknowledgements}

Single channel recordings were performed in collaboration with the group of Mathias Winterhalter at Jacobs University Bremen in Germany.

\section{References}

(1) Danzer, K. M., Haasen, D., Karow, A. R., Moussaud, S., Habeck, M., Giese, A., Kretzschmar, H., Hengerer, B., and Kostka, M. (2007) Different species of alpha-synuclein oligomers induce calcium influx and seeding. J. Neurosci. 27, 9220-32.

(2) Kayed, R., Head, E., Thompson, J. L., McIntire, T. M., Milton, S. C., Cotman, C. W., and Glabe, C. G. (2003) Common structure of soluble amyloid oligomers implies common mechanism of pathogenesis. Science 300, 486-489.

(3) Kayed, R., Sokolov, Y., Edmonds, B., McIntire, T. M., Milton, S. C., Hall, J. E., and Glabe, C. G. (2004) Permeabilization of lipid bilayers is a common conformation-dependent activity of soluble amyloid oligomers in protein misfolding diseases. J. Biol. Chem. 279, 46363-46366.

(4) Lashuel, H. A., Hartley, D., Petre, B. M., Walz, T., and Lansbury, P. T., Jr. (2002) Neurodegenerative disease: amyloid pores from pathogenic mutations. Nature 418, 291. 
(5) Volles, M. J., Lee, S. J., Rochet, J. C., Shtilerman, M. D., Ding, T. T., Kessler, J. C., and Lansbury, P. T., Jr. (2001) Vesicle permeabilization by protofibrillar alpha-synuclein: implications for the pathogenesis and treatment of Parkinson's disease. Biochemistry 40, 7812-7819.

(6) Zhu, M., Li, J., and Fink, A. L. (2003) The association of alphasynuclein with membranes affects bilayer structure, stability, and fibril formation. J. Biol. Chem. 278, 40186-40197.

(7) Volles, M. J., and Lansbury, P. T., Jr. (2002) Vesicle permeabilization by protofibrillar alpha-synuclein is sensitive to Parkinson's disease-linked mutations and occurs by a pore-like mechanism. Biochemistry 41, 4595-4602.

(8) Quist, A., Doudevski, I., Lin, H., Azimova, R., Ng, D., Frangione, B., Kagan, B., Ghiso, J., and Lal, R. (2005) Amyloid ion channels: a common structural link for protein-misfolding disease. Proc. Natl. Acad. Sci. U. S. A. 102, 10427-10432.

(9) Lashuel, H. A., Petre, B. M., Wall, J., Simon, M., Nowak, R. J., Walz, T., and Lansbury, P. T., Jr. (2002) Alpha-synuclein, especially the Parkinson's disease-associated mutants, forms porelike annular and tubular protofibrils. J. Mol. Biol. 322, 10891102.

(10) Shai, Y. (1999) Mechanism of the binding, insertion and destabilization of phospholipid bilayer membranes by alpha-helical antimicrobial and cell non-selective membrane-lytic peptides. BBABiomembranes 1462, 55-70.

(11) van Rooijen, B. D., Claessens, M. M. A. E., and Subramaniam, V. (2009) Lipid bilayer disruption by oligomeric alpha-synuclein depends on bilayer charge and accessibility of the hydrophobic core. Biochim. Biophys. Acta - Biomembr. 1788, 1271-1278.

(12) Montal, M., and Mueller, P. (1972) Formation of bimolecular membranes from lipid monolayers and a study of their electrical properties. Proc. Natl. Acad. Sci. U. S. A. 69, 3561-3566.

(13) Qin, F. (2004) Restoration of single-channel currents using the segmental k-means method based on hidden Markov modeling. Biophys. J. 86, 1488-1501.

(14) Ladokhin, A. S., Wimley, W. C., and White, S. H. (1995) Leakage of membrane vesicle contents: Determination of mechanism using fluorescence requenching. Biophys. J. 69, 1964-1971.

(15) van Rooijen, B. D., Claessens, M. M. A. E. , and Subramaniam, V. (2008) Membrane binding of oligomeric alpha-synuclein depends on bilayer charge and packing. FEBS Lett. 582, 3788-3792. 
(16) Valincius, G., Heinrich, F., Budvytyte, R., Vanderah, D. J., McGillivray, D. J., Sokolov, Y., Hall, J. E., and Lösche, M. (2008) Soluble Amyloid beta-Oligomers Affect Dielectric Membrane Properties by Bilayer Insertion and Domain Formation: Implications for Cell Toxicity. Biophys. J. 95, 4845-4861.

(17) Capone, R., Quiroz, F. G., Prangkio, P., Saluja, I., Sauer, A. M., Bautista, M. R., Turner, R. S., Yang, J., and Mayer, M. (2009) Amyloid-beta-Induced Ion Flux in Artificial Lipid Bilayers and Neuronal Cells: Resolving a Controversy. Neurotox. Res. 16, $1-13$.

(18) Shoemaker, S. D., and Vanderlick, T. K. (2002) Intramembrane electrostatic interactions destabilize lipid vesicles. Biophys. J. 83, 2007-2014.

(19) Melikov, K. C., Frolov, V. A., Shcherbakov, A., Samsonov, A. V., Chizmadzhev, Y. A., and Chernomordik, L. V. (2001) Voltageinduced nonconductive pre-pores and metastable single pores in unmodified planar lipid bilayer. Biophys. J. 80, 1829-1836.

(20) Chen, P. S., Toribara, T. Y., and Warner, H. (1956) Microdetermination of Phosphorus. Anal. Chem. 28, 1756-1758. 



\section{Chapter 8 \\ Conclusions, summary and outlook}

Almost a decade ago it was suggested that oligomeric $\alpha \mathrm{S}$ could be a toxic intermediate in the aggregation of $\alpha \mathrm{S}$ (1). Membrane permeabilization by these oligomeric species was subsequently proposed as a likely mechanism of toxicity (2). A relatively small number of studies have since established supporting evidence for this hypothesis. Regardless, or perhaps because of this small body of evidence, oligomer membrane permeabilization remains a hotly debated subject and is often referred to as a possible mechanism of toxicity in PD. This might also be related to the evidence that in many amyloid diseases, aggregation intermediates have been found to possess toxic properties and to permeabilize lipid membranes. The "amyloid pore hypothesis" seems to allow for a unifying explanation for the onset and progression of these complex amyloid diseases (3). For some of the amyloid proteins there is convincing evidence that the aggregation intermediates are toxic through membrane permeabilization (4). In the case of $\alpha \mathrm{S}$ and Parkinson's disease, the toxicity and membrane interaction of oligomeric intermediates is not well characterized. Therefore, the aim of this thesis was to elucidate how oligomeric $\alpha \mathrm{S}$ interacts with lipid membranes.

In order to characterize oligomer-membrane interactions one has to prepare $\alpha \mathrm{S}$ oligomers. For biophysical or biochemical characterization, a stable homogeneous solution of oligomeric $\alpha \mathrm{S}$ is preferred. The aggregation process of $\alpha \mathrm{S}$ and especially the first steps during oligomerization are however not understood. Instead of fully exploring the early stages of aggregation and pinpointing exactly which oligomeric intermediate is most toxic, we have taken a different approach. Under specific conditions, stable $\alpha \mathrm{S}$ oligomers can be obtained that are known to interact with lipids (2). Using a similar approach we have incubated $\alpha \mathrm{S}$ at high concentration to obtain a stable and relatively homogeneous population of oligomeric $\alpha \mathrm{S}$. Our initial results showed that our preparation method resulted in morphologically similar oligomers with comparable vesicle disruptive properties to those reported in the literature $(5,6)$. We have cho- 
sen to fully characterize the interaction with lipids of this specific oligomeric species. This is an important point and the conclusions presented in this thesis are only valid for this particular oligomeric species. One could always oppose generalization of these findings by stating that we are simply not examining the relevant oligomeric intermediate. However, we believe that even in the case that the true toxic oligomer differs from the one described in this thesis, the work presented here might prove extremely useful. We have established techniques and methods to study these intermediates that are generally applicable, irrespective of the specific characteristics of the oligomeric species being studied. In addition, comparing the biophysical and biochemical properties of a toxic to a non-toxic oligomeric species might actually give important insights into the mechanisms of toxicity and the structural basis for this toxicity.

\section{Oligomer structure}

The structure of $\alpha \mathrm{S}$ oligomers is experimentally not easily accessible by standard structural biology methods. Therefore, there is no detailed structural information on the possible conformation of oligomeric $\alpha \mathrm{S}$. The results presented in this thesis contain some structural implications for the specific $\alpha \mathrm{S}$ oligomeric species that were created.

The oligomers have a hydrodynamic radius of around 7.2 to $9.3 \mathrm{~nm}$ as estimated by fluorescence correlation spectroscopy. The molecular weight or the number of $\alpha \mathrm{S}$ monomers in the oligomeric complex depends on the packing density of the oligomer. Tryptophan fluorescence quenching by acrylamide and ANS dye binding experiments suggest that the oligomers are not as densely packed as globular proteins. Assuming the oligomers have a similar packing density as a molten globule structure (7), the oligomer consists of around 40 to 80 monomers. Electron microscopy and atomic force microscopy imaging on dried protein samples show spherical particles up to 8 and $11 \mathrm{~nm}$ in diameter respectively. Donut shaped particles have been observed by electron microscopy; however, more careful and systematic experiments are needed to conclude such structural details from an imaging method.

The oligomeric aggregates share some properties with fibrillar $\alpha \mathrm{S}$. They are quite large, and circular dichroism spectroscopy indicates a $\beta$-sheet-like structure. Tryptophan spectroscopy and quenching studies reveal that for oligomeric $\alpha \mathrm{S}$ especially the residues $\sim 4-90$ are in a considerably more protected and apolar environment compared to monomeric $\alpha \mathrm{S}$. The highly negatively charged C-terminus of the protein is the most accessible part in the oligomeric form and more likely to be solvent exposed. Fibrils are organized in a similar fashion; the hydrophobic core region of the protein is protected whereas the C-terminus is more exposed $(8,9)$. Similar to the fibrils, the oligomers are 
stable and do not readily dissociate into monomeric $\alpha \mathrm{S}$. However, oligomers are not merely small fibrils. The dye thioflavin- $\mathrm{T}$ that reports on amyloid structure did not show a considerable increase in fluorescence when added to the oligomers. The oligomers also did not seed the aggregation of monomeric $\alpha \mathrm{S}$ efficiently while fragmented fibrils do. The oligomers are thus most likely off-pathway from the aggregation into amyloid fibrils. The oligomers appear to be limited in their growth since monomer-oligomer binding by fluorescence correlation spectroscopy was indistinguishable from the background signal.

The elongation and growth of $\alpha \mathrm{S}$ fibrils is possible because the monomer that is added to the structure presents a new open or active end to which an additional monomer can bind. The assembly of the oligomers is likely governed by the same physical driving forces. Hydrogen bonding, formation of $\beta$-sheet structure and collapse of the hydrophobic regions may stabilize the oligomer. However, the oligomeric aggregate is not as well ordered as the fibrillar structure. Therefore, at a certain size of the oligomers, there might be no open ends which are presented to solution and the growth of the oligomer is slowed down. A possible way in which these oligomers can still form amyloid is if they reorganize into the amyloid fold. Simulation studies suggest that this is a possible mechanism through which the amyloid structure is formed (10). Off-pathway oligomers then just represent aggregates that are not able to reorganize. This could either be related to their size (larger particles may be less efficient in reorganizing) or to different possible conformations at very early stages (dimers, trimers).

\section{Oligomer lipid binding}

Before a protein can form a pore-like complex in the bilayer or otherwise cause membrane permeabilization, the protein has to bind the membrane. The membrane binding of monomeric $\alpha \mathrm{S}$ is well characterized in literature (11). Monomeric $\alpha \mathrm{S}$ binds to negatively charged lipids primarily through electrostatic interactions (12). Lipid binding is mediated by the N-terminal residues 1-100, which fold into an amphipathic helix that lies flat on the membrane (13). How the lipid binding of $\alpha \mathrm{S}$ changes upon aggregation is not known. Therefore, we have characterized the lipid binding properties of fluorescently labeled oligomeric $\alpha \mathrm{S}$, using confocal microscopy and fluorescence correlation spectroscopy.

Similar to the monomeric protein, the membrane binding of oligomeric $\alpha \mathrm{S}$ depends on the charge of the membrane. The oligomers bind to membranes composed of negatively charged phospholipids in the liquid disordered phase irrespective of the exact chemical structure of the lipid headgroup. The binding affinity of oligomeric $\alpha \mathrm{S}$ to large unilamellar vesicles composed of phos- 
phatidylserine is slightly lower compared to monomeric $\alpha \mathrm{S}$. Addition of the zwitterionic lipid phosphatidylcholine to the membrane greatly reduces the membrane binding of oligomeric $\alpha \mathrm{S}$. The oligomers do not bind to pure phosphatidylcholine membranes.

Although the specific binding to negatively charged membranes suggests a role for electrostatic interactions, binding is inhibited at low ionic strength. This can be explained by the high net negative charge of the protein. In the absence of charge screening, the penalty of bringing the negatively charged aggregate to the membrane might be too large. In addition, the conformational flexibility of the oligomer might be reduced compared to the monomeric protein. So a conformational change to keep the C-terminus away from the bilayer may be partly restricted, which could explain the lower lipid affinity of oligomeric $\alpha \mathrm{S}$ compared to the monomeric protein. The binding specificity to negatively charged phospholipids suggests that the N-terminal part of the protein, which contains several positively charged and hydrophobic residues, might be important in lipid binding.

Tryptophan fluorescence spectroscopy indeed shows that the N-terminus is involved in oligomer-lipid binding. However, the results also indicate that these residues are in a solvent protected apolar environment. There must be some conformational flexibility in the $\alpha \mathrm{S}$ oligomers to allow these residues to interact with the membrane, and to maximize the favorable interactions. How these structural features relate to the lipid permeabilization properties of the oligomers is yet unclear. Information on how deep the oligomer inserts into the bilayer structure would for instance be extremely helpful.

\section{Membrane permeabilization}

Although the permeabilization and disruption of cellular membranes by oligomeric $\alpha \mathrm{S}$ has been suggested as a possible cytotoxic process (3), the exact mechanism by which this occurs is not well established. A pore-like model in which the oligomeric complexes form distinct trans-membrane pores has been suggested but this model is supported by little evidence $(6,14)$.

We have tried to characterize the disruption mechanism of oligomeric $\alpha \mathrm{S}$ in different in vitro lipid bilayer systems. The oligomers cause efflux of the dye calcein when added to vesicles composed of negatively charged lipids. When the vesicles are prepared from 1:1 mixtures of a zwitterionic lipid with a negatively charged lipid, vesicle disruption is generally inhibited. Under these conditions oligomers are still able to bind the membrane, highlighting that membranebound oligomers do not always induce membrane permeabilization.

To determine the mechanism by which the membrane is permeabilized, several different assays were performed. Single channel-like events were observed 
Conclusions, summary and outlook

in a planar lipid bilayer experiment. However, the results were very irreproducible partly because planar bilayers of the negatively charged lipid are intrinsically unstable. Therefore, large and giant unilamellar vesicles were used as a membrane system to determine the mechanistic aspects of oligomer-membrane disruption. Dye efflux from vesicles induced by oligomeric $\alpha \mathrm{S}$ is fast and occurs through an all-or-none mechanism. The vesicles are not completely destroyed or solubilized. Both dynamic light scattering and confocal microscopy indicate that vesicle morphology remains roughly unchanged. The membrane disruption of these purely negatively charged vesicles does not seem to occur through a pore-like mechanism. Even $70 \mathrm{kDa}$ dextran molecules were able to escape the vesicles interior upon addition of oligomeric $\alpha \mathrm{S}$.

Possibly, disruption of the membrane integrity is related to an intrinsic instability of vesicles from negatively charged lipids. Several unrelated proteins induced dye efflux when added in sufficient amounts to these vesicles. The membrane instability caused by the adsorption of the protein could lead to large defects in the membrane. However, compared to other proteins and also monomeric $\alpha \mathrm{S}$, the $\alpha \mathrm{S}$ oligomers are very efficient in destabilizing the membrane. This destabilization is thus related to structural features of the oligomeric complex.

We cannot completely exclude the possibility that the oligomers have porelike properties. In planar lipid bilayers single channel-like events are sometimes observed. Additionally, in the presence of calcium, the membrane permeability is sensitive to the marker size. The $3 \mathrm{kDa}$ dextran conjugate could not escape the vesicle interior whereas the quencher DPX (422 Da) could cross the membrane in the presence of oligomeric $\alpha \mathrm{S}$. Vesicle permeability is not transient and persists even an hour after addition of the $\alpha \mathrm{S}$ oligomers and can therefore not be attributed solely to transient non-equilibrium processes. However, also in the presence of calcium, non-equilibrium processes have a major contribution to the permeabilization process, which points to the presence of defects in the bilayer.

\section{Relevance}

The results presented in this thesis give an insight into how in vitro generated $\alpha \mathrm{S}$ oligomers interact with synthetic phospholipid systems. One of the biggest questions that arises is if the process of lipid disruption by $\alpha \mathrm{S}$ oligomeric species could occur in biological systems as well. The results from our experiments can only provide a limited answer to this question. The intracellular milieu is far more complex than the test tube environment. Biological membranes contain a large quantity of different lipids and are rich in protein, and the aqueous space is crowded with macromolecules. Both the conformation of the possible 
intracellular $\alpha \mathrm{S}$ oligomers, as well as the properties of membranes, could be different. Nevertheless, the results presented here give insights into how $\alpha \mathrm{S}$ oligomers might interact with membranes inside neurons.

Our results clearly show that $\alpha \mathrm{S}$ oligomers can interact with lipid membranes. The lipid binding ability of the protein is not abolished upon aggregation. Given the lipid binding properties of the $\alpha \mathrm{S}$ oligomers, intracellular membranes that contain some percentage of negatively charged lipids could be a target for oligomer binding. However, membrane permeabilization only occurs in membranes containing a large fraction of negatively charged lipids. These high concentrations are generally not found inside the cell (15). Since lipids are not evenly distributed throughout the cell, local concentrations of anionic lipids can be quite high. For instance the inner leaflet of the plasma membrane might contain up to $30 \%$ of phosphatidylserine $(15,16)$, and the inner mitochondrial membrane contains around $25 \%$ of cardiolipin (17). The concentrations of anionic lipids in the cell appear not to be high enough to be vulnerable to oligomer membrane disruption. However, we can not exclude the possibility that lipid segregation or domain formation might induce higher local concentrations of anionic lipids. Most likely however, the oligomers we prepared in this work are not likely to permeabilize real biological membranes.

The in vitro vesicle permeabilization properties of oligomeric $\alpha \mathrm{S}$ are often regarded as supporting evidence for the relevance of the amyloid pore hypothesis to Parkinson's disease. However, this is only a valid argument if results from such biophysical experiments are followed up by experiments on real biological systems. Our data highlight that in vitro vesicle disruption may not be a good predictor of in vivo activity and toxicity. How $\alpha \mathrm{S}$ oligomers interact with real biological membranes thus remains an important open question.

\section{Outlook}

Unraveling the pathogenesis of Parkinson's disease has proven to be extremely challenging since the initial discovery of the disease almost 200 years ago. It is still not known what causes the neuronal cell loss in the substantia nigra and other parts of the brain. Many changes are linked to the Parkinsonian brain, such as inflammation, oxidative stress and protein aggregation. However, it is often difficult to elucidate if these changes are a cause or an effect of neuronal stress. The role of $\alpha \mathrm{S}$ aggregation in Parkinson's disease is therefore still not clear. In addition, remarkably little is known about the normal biological function of $\alpha \mathrm{S}$. So there are still many important outstanding questions that need to be answered by biology.

Although a biochemical and biophysical characterization of $\alpha \mathrm{S}$ may not directly answer such questions, fundamental knowledge on $\alpha \mathrm{S}$ structure and 
properties could contribute to a better understanding of the cell biology of $\alpha \mathrm{S}$. In addition to its relevance in disease, $\alpha \mathrm{S}$ also serves as a model system of protein aggregation as well as for the class of intrinsically disordered proteins. For these reasons, $\alpha \mathrm{S}$ has been intensely studied over the past decade. However, there are still important open questions for the field to answer.

Resolving the early steps of $\alpha \mathrm{S}$ aggregation is one of the biggest challenges that need to be tackled. Although the fibrillar structure at the end stage of aggregation is relatively well understood, it is not known how these are formed. What are the structures of the initial aggregation intermediates? Does this structure change with increasing size of the aggregate? Are different structural conformers possible for the same number of monomers? How do these structures influence the stability and the propensity to associate with new $\alpha \mathrm{S}$ monomers? Answering these questions will not be easy. Intermediates might be heterogeneous, unstable and only present at low concentrations.

Once intermediates have been structurally characterized, it would be interesting to see how these structural characteristics mediate the interaction with other components such as lipid bilayers. Do intermediates exist that possess different lipid binding properties than the monomeric protein? Is there an intermediate which indeed forms pore-like structures in the membrane? Lipid bilayers could also be used to selectively "fish out" aggregates with different lipid binding properties. It has also been suggested that the membrane itself could act as an aggregation matrix which could lead to structurally different oligomers or induce $\alpha \mathrm{S}$ aggregation at lower absolute concentrations of the protein. Besides membranes, there are of course other cellular components, such as the proteosome, that could interact with oligomeric species.

Finally, the question remains if there exists a distinct toxic species of $\alpha \mathrm{S}$. Proving cytotoxic properties of $\alpha \mathrm{S}$ aggregation intermediates is often done by adding the oligomers extracellularly to cultured cells. Since $\alpha \mathrm{S}$ is an intracellular protein this might not be the most relevant toxicity assay. The opposite approach is to induce $\alpha \mathrm{S}$ overexpression and wait for toxicity or aggregation to occur inside the cell. However, in addition to the question if systems relying on protein overexpression are relevant, the tools for assessing the aggregation state and conformation of $\alpha \mathrm{S}$ inside a cell are severely limited.

It seems that after a decade of $\alpha \mathrm{S}$ research, although significant steps have been made, there is still no good understanding of how this protein modulates neuronal toxicity in Parkinson's disease. Although both structurally and functionally the protein has been the matter of intense research, there are still many questions left to which biophysical research can contribute. The problems that remain are indisputably relevant, but might be extremely challenging to solve. 


\section{References}

(1) Conway, K. A., Lee, S. J., Rochet, J. C., Ding, T. T., Williamson, R. E., and Lansbury, P. T., Jr. (2000) Acceleration of oligomerization, not fibrillization, is a shared property of both alpha-synuclein mutations linked to early-onset Parkinson's disease: implications for pathogenesis and therapy. Proc. Natl. Acad. Sci. U. S. A. 97, 571-576.

(2) Volles, M. J., Lee, S. J., Rochet, J. C., Shtilerman, M. D., Ding, T. T., Kessler, J. C., and Lansbury, P. T., Jr. (2001) Vesicle permeabilization by protofibrillar alpha-synuclein: implications for the pathogenesis and treatment of Parkinson's disease. Biochemistry 40, 7812-7819.

(3) Kayed, R., Head, E., Thompson, J. L., McIntire, T. M., Milton, S. C., Cotman, C. W., and Glabe, C. G. (2003) Common structure of soluble amyloid oligomers implies common mechanism of pathogenesis. Science 300, 486-489.

(4) Lashuel, H. A., and Lansbury, P. T. (2006) Are amyloid diseases caused by protein aggregates that mimic bacterial pore-forming toxins? Q. Rev. Biophys. 39, 167-201.

(5) Lashuel, H. A., Petre, B. M., Wall, J., Simon, M., Nowak, R. J., Walz, T., and Lansbury, P. T., Jr. (2002) Alpha-synuclein, especially the Parkinson's disease-associated mutants, forms porelike annular and tubular protofibrils. J. Mol. Biol. 322, 10891102 .

(6) Volles, M. J., and Lansbury, P. T., Jr. (2002) Vesicle permeabilization by protofibrillar alpha-synuclein is sensitive to Parkinson's disease-linked mutations and occurs by a pore-like mechanism. Biochemistry 41, 4595-4602.

(7) Uversky, V. N. (2002) What does it mean to be natively unfolded? Eur. J. Biochem. 269, 2-12.

(8) Del Mar, C., Greenbaum, E. A., Mayne, L., Englander, S. W., and Woods, V. L. (2005) Structure and properties of alpha-synuclein and other amyloids determined at the amino acid level. Proc. Natl. Acad. Sci. U. S. A. 102, 15477-15482.

(9) Miake, H., Mizusawa, H., Iwatsubo, T., and Hasegawa, M. (2002) Biochemical characterization of the core structure of alphasynuclein filaments. J. Biol. Chem. 277, 19213-19219. 
(10) Cheon, M., Chang, I., Mohanty, S., Luheshi, L. M., Dobson, C. M., Vendruscolo, M., and Favrin, G. (2007) Structural reorganisation and potential toxicity of oligomeric species formed during the assembly of amyloid fibrils. PlOs Comput. Biol. 3, 1727-1738.

(11) Beyer, K. (2007) Mechanistic aspects of Parkinson's disease: alpha-synuclein and the biomembrane. Cell Biochem. Biophys. 47, 285-299.

(12) Rhoades, E., Ramlall, T. F., Webb, W. W., and Eliezer, D. (2006) Quantification of alpha-synuclein binding to lipid vesicles using fluorescence correlation spectroscopy. Biophys. J. 90, 4692-4700.

(13) Eliezer, D., Kutluay, E., Bussell, R., Jr., and Browne, G. (2001) Conformational properties of alpha-synuclein in its free and lipidassociated states. J. Mol. Biol. 307, 1061-1073.

(14) Quist, A., Doudevski, I., Lin, H., Azimova, R., Ng, D., Frangione, B., Kagan, B., Ghiso, J., and Lal, R. (2005) Amyloid ion channels: a common structural link for protein-misfolding disease. Proc. Natl. Acad. Sci. U. S. A. 102, 10427-10432.

(15) van Meer, G., Voelker, D. R., and Feigenson, G. W. (2008) Membrane lipids: where they are and how they behave. Nat. Rev. Mol. Cell Biol. 9, 112-124.

(16) Devaux, P. F. (1991) Static And Dynamic Lipid Asymmetry In Cell-Membranes. Biochemistry 30, 1163-1173.

(17) Daum, G. (1985) Lipids Of Mitochondria. Biochim. Biophys. Acta $822,1-42$. 



\section{Appendix A \\ Fluorescence correlation spectroscopy}

\section{FCS principle}

In FCS the fluorescence emission from fluorescent molecules in a small confocal volume is measured. To achieve this, the laser beam for excitation is focused through a high numerical aperture objective into a diffraction limited spot. Fluorescence emission is collected though the same objective and out of focus light is blocked by focusing on a confocal pin hole. The resulting confocal volume has a typical lateral dimension of $\sim 500 \mathrm{~nm}$ and $\sim 2 \mu \mathrm{m}$ in depth, yielding a volume of $\sim 0.5-1 \mathrm{fl}$. If the concentration of fluorescent molecules is low enough, individual fluorophores diffusing through the confocal volume will give rise to substantial fluctuations in the observed fluorescence emission. The characteristic time constants in these fluctuations are representative of the diffusion properties of the fluorophore and can be extracted from the signal by calculating the autocorrelation function $G(\tau)$ :

$$
G(\tau)=\frac{\langle\delta F(t) \delta F(t+\tau)\rangle}{\langle F(t)\rangle^{2}}
$$

where $F(t)$ is the fluorescence emission, $\delta F(t)=F(t)-\langle F(t)\rangle$ is the fluctuation in the fluorescence signal with respect to the average fluorescence signal, and $\tau$ is the time shift. To extract the diffusion time through the focus of the fluorophore from the autocorrelation curves, a three dimensional free diffusion model for a single freely diffusing species was fitted to the data:

$$
G(\tau)=\frac{1}{N}\left(\left(1+\frac{\tau}{\tau_{i}}\right) \sqrt{1+\omega^{2} \frac{\tau}{\tau_{i}}}\right)^{-1}
$$

where $N$ is the average number of particles in the focal volume, $\tau_{i}$ is the diffusion time through the focus of the fluorescent molecule, and $\omega$ represents the ratio of the radial to axial dimensions of the confocal volume. The parameter $\omega$ can be determined from fitting the correlation curves of free AL488 and was subsequently fixed during the rest of the experiments.

\section{Molecular weight determination}

The diffusion constant of the fluorophore can be determined from the diffusion time through the focus, if the dimensions of the confocal volume are known. 
These can be calibrated by using a fluorophore which has a known diffusion time. The diffusion time of AL488 has been reported in literature (1) and this value was used to calibrate the confocal volume. From the diffusion time the hydrodynamic radius $r$ of the particle can be calculated through the StokesEinstein relation:

$$
D=\frac{k_{B} T}{6 \pi \nu r}
$$

where $D$ is the diffusion constant, $k_{B}$ is the Boltzmann's constant, $T$ is the temperature, and $\nu$ is the viscosity. For proteins the hydrodynamic radius can be related to the molecular weight of the protein if the packing density of the protein is known (2).

\section{Binding experiments}

Monomer-oligomer binding can be measured using FCS since the oligomers have a much larger diffusion time than the monomeric protein $(3,4)$. In the binding experiments the correlation curves are fit with a two component free diffusion model:

$$
\begin{aligned}
G(\tau)= & \frac{1}{N}\left(f\left(\left(1+\frac{\tau}{\tau_{b}}\right) \sqrt{1+\omega^{2} \frac{\tau}{\tau_{i}}}\right)^{-1}\right. \\
& \left.+(1-f)\left(\left(1+\frac{\tau}{\tau_{f}}\right) \sqrt{1+\omega^{2} \frac{\tau}{\tau_{i}}}\right)^{-1}\right)
\end{aligned}
$$

where $f$ is the fraction of oligomer-bound monomer, $\tau_{b}$ and $\tau_{f}$ are the diffusion times of the bound and free monomer respectively. In a similar fashion oligomer binding to LUVs can be measured, since the vesicles have a much larger diffusion time than the oligomeric species.

\section{Practical realization}

FCS measurements were performed on a confocal fluorescence microscope described in detail elsewhere (5). Briefly the $488 \mathrm{~nm}$ laser line of an $\mathrm{Ar}+/ \mathrm{Kr}+$ gas laser was used to excite AL488 with a laser power of around $30 \mu \mathrm{W}$ just before entering the objective (Zeiss $\mathrm{C}$-Apochromat $63 \times, \mathrm{NA}=1.20$ ). Fluorescence emission was detected with an avalanche photo diode. The counts were binned with a $12.5 \mu \mathrm{s}$ bin time and FCS analysis was subsequently performed off line. For each datapoint 25 correlation curves of 15 seconds each were recorded and averaged. 


\section{References}

(1) Nitsche, J. M., Chang, H. C., Weber, P. A., and Nicholson, B. J. (2004) A transient diffusion model yields unitary gap junctional permeabilities from images of cell-to-cell fluorescent dye transfer between Xenopus oocytes. Biophys. J. 86, 2058-2077.

(2) Uversky, V. N. (2002) What does it mean to be natively unfolded? Eur. J. Biochem. 269, 2-12.

(3) Rusu, L., Gambhir, A., McLaughlin, S., and Rädler, J. (2004) Fluorescence correlation spectroscopy studies of Peptide and protein binding to phospholipid vesicles. Biophys. J. 87, 1044-1053.

(4) Rhoades, E., Ramlall, T. F., Webb, W. W., and Eliezer, D. (2006) Quantification of alpha-synuclein binding to lipid vesicles using fluorescence correlation spectroscopy. Biophys. J. 90, 4692-4700.

(5) Zhang, D., Lans, H., Vermeulen, W., Lenferink, A., and Otto, C. (2008) Quantitative fluorescence correlation spectroscopy reveals a 1000-fold increase in lifetime of protein functionality. Biophys. J. 95, 3439-3446. 



\section{Appendix B Phospholipid structure}

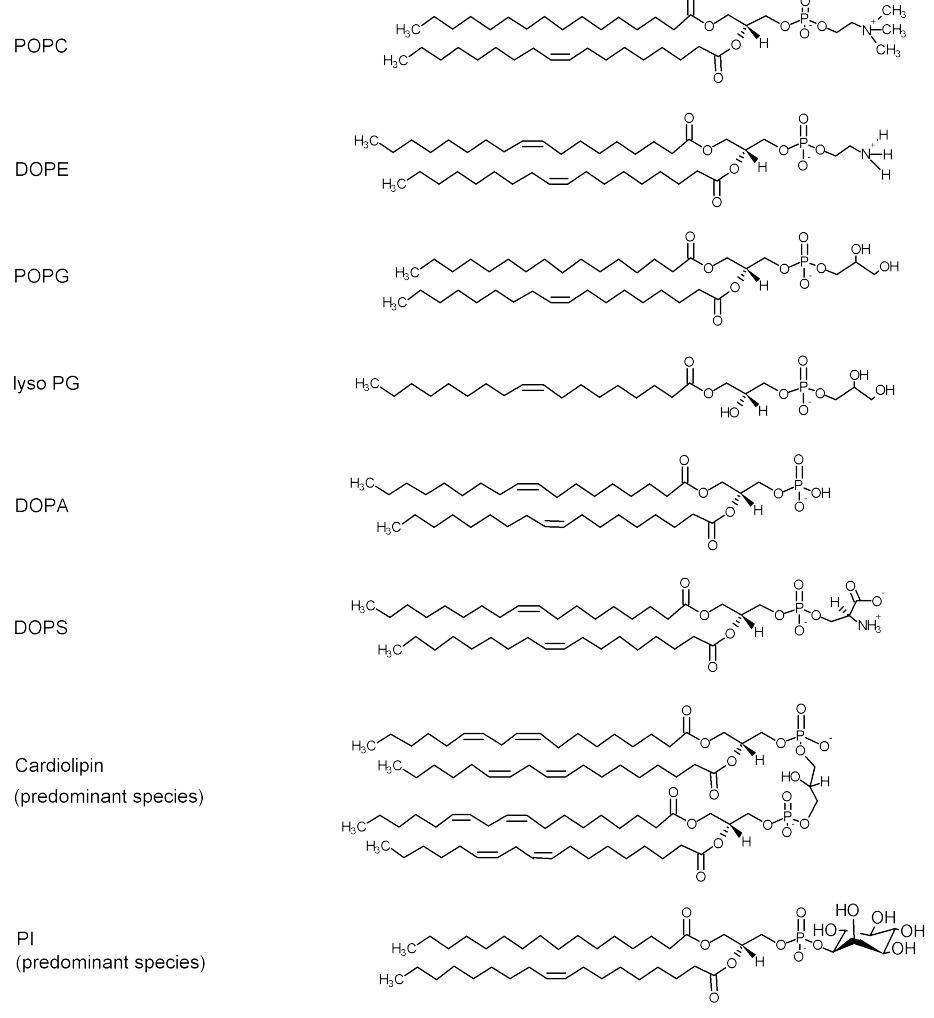





\section{Abbreviations}

18:2 PG 1,2-Dilinoleoyl phosphatidylglycerol

AFM Atomic force microscopy

AL488 Alexa 488

ANS 8-Anilino-1-naphthalenesulfonic acid

$\alpha \mathrm{S} \quad \alpha$-Synuclein

Bis-ANS 4,4-Dianilino-1,1-binaphthyl-5,5-disulfonic acid

CD Circular dichroism

CL Cardiolipin

chol Cholesterol

DCVJ 9-(2,2-Dicyanovinyl)julolidine

DLS Dynamic light scattering

DOPA 1,2-Dioleoyl phosphatidic acid

DOPC 1,2-Dioleoyl phosphatidylcholine

DOPE 1,2-Dioleoyl phosphatidylethanolamine

DOPG 1,2-Dioleoyl phosphatidylglycerol

DOPS 1,2-Dioleoyl phosphatidylserine

DPPG 1,2-Dipalmitoyl phosphatidylglycerol

DPX p-Xylene-bis(N-pyridinium bromide)

DTT DL-Dithiothreitol

FCS Fluorescence correlation spectroscopy

GUV Giant unilamellar vesicle

HPTS 8-Hydroxypyrene-1,3,6-trisulfonic acid

IDP Intrinsically disordered protein

Ld Liquid disordered

Lo Liquid ordered

LUV Large unilamellar vesicle

lyso PG 1-Oleoyl phosphatidylglycerol

NATA N-Acetyl-L-tryptophanamide

NPN N-Phenyl-1-naphthylamine

PAGE Polyacrylamide gel electrophoresis

PD Parkinson's disease

PI L- $\alpha$-phosphatidylinositoL

POPC 1-Palmitoyl, 2-oleoyl phosphatidylcholine

POPG 1-Palmitoyl, 2-oleoyl phosphatidylglycerol

POPS 1-Palmitoyl, 2-oleoyl phosphatidylserine 
PTS 8-Isothiocyanatopyrene-1,3,6-trisulfonic acid

Rhod Lissamine Rhodamine B

ThioT Thioflavin-T

TNS 2-(p-Toluidino)-6-naphthalenesulfonic acid

Trp Tryptophan

UBS Ubiquitin-proteasome system

wt Wild-type 


\section{Summary}

Parkinson's disease is a neurodegenerative disease common with old age. The neuronal protein $\alpha$-synuclein is considered to play a pivotal role in the onset and progression of the disease. The protein is the main constituent of the Lewy body, the pathological hallmark of the disease, which are large intracellular inclusion bodies that contain aggregated fibrillar protein. In addition mutations in the gene coding for the protein as well as gene multiplications lead to genetic variants of Parkinson's disease. One of the main questions is how the aggregation of $\alpha$-synuclein is related to the disease. Recent evidence suggests that oligomeric intermediates in the aggregation process of $\alpha$-synuclein are potentially the neurotoxic species that underly the disease. A potential mechanism by which these could be toxic is through interaction and disruption of cellular membranes. In this thesis, we try to elucidate how oligomeric $\alpha$-synuclein interacts with lipid membranes.

In Chapter 2 the $\alpha$-Synuclein oligomers that were prepared in vitro are characterized. The purified oligomers are stable and contain around 40-80 monomers of the protein. Although the oligomeric aggregates contain $\beta$-sheet content they are structurally distinct from fibrils formed by $\alpha$-synuclein. The oligomers are most likely off-pathway from fibril formation since aggregation cannot be seeded by the oligomers.

In Chapter 3 the vesicle permeabilization properties of $\alpha$-synuclein are characterized. $\alpha$-Synuclein oligomeric species can disrupt lipid membranes. They induce content efflux from lipid vesicles, which indicates a loss of bilayer integrity. The vesicle permeabilization is primarily dependent on the charge of the membrane. Only vesicles composed of negatively charged lipids were disrupted. In addition, membrane packing also modulates the permeabilization. More loosely packed membranes were more prone to disruption by the oligomers.

Chapter 4 and 5 describe the lipid binding properties of oligomeric $\alpha$ synuclein. The specificity of the membrane permeabilization process for negatively charged lipids is primarily caused by the lipid binding properties of oligomeric $\alpha$-synuclein. Fluorescently labeled oligomers only co-localize with the membranes of vesicles if the membranes contains negatively charged lipids. However, membrane bound oligomers do not lead to a permeabilized membrane under all conditions. The oligomers bind to negatively charged vesicles with a slightly lower affinity compared to the monomeric protein with a micromolar 
dissociation constant. The affinity for membrane binding drops with decreasing anionic lipid content. The binding properties suggest that the oligomers have structural elements such as charged patches or a conformational flexibility, that support the lipid binding.

In Chapter 6 the structure of the oligomers is assessed using tryptophan fluorescence. The lipid binding properties of oligomeric $\alpha$-synuclein must be caused by the structural properties of the oligomers. The specificity for negatively charged lipids suggests that the positively charged N-terminus of the protein is involved in membrane binding. The results from tryptophan fluorescence spectroscopy on mutant $\alpha$-synuclein containing a tryptophan residue at different positions, suggest that indeed the N-terminus mediates the lipid binding of the oligomers. In addition, the C-terminus of the protein is solvent exposed in the oligomeric particle, whereas the N-terminus makes up the core of the aggregate.

Chapter 7 tries to elucidate the mechanism by which oligomeric $\alpha$-synuclein permeabilizes the membrane. How membrane bound $\alpha$-synuclein disrupts the bilayer integrity is not clear. A pore-like model has been suggested, in which the oligomers form distinct structures in the membrane through which solutes can diffuse. Although we cannot unequivocally confirm or oppose this model, our data indicates that membrane instability is an important factor. Negatively charged vesicles are intrinsically unstable to protein adsorption. Large bilayer defects caused by membrane bound proteins cause a high permeability of the membrane. Even under conditions that stabilize the negatively charged vesicles, evidence for defect formation was observed.

Although we now have a much better understanding how oligomeric $\alpha$ synuclein interacts with lipid membranes, the actual relevance of this process to Parkinson's disease is still not clear. In addition to the amyloid pore hypothesis we have worked on in this thesis, many alternative promising models for the mechanistic aspects of the disease exist. However, determining the mechanisms underlying Parkinson's disease is extremely complex. Research into the problem of protein aggregation and its role in disease has been ongoing intensively for the past decade. However, even basic question as: Does the aggregation of $\alpha$-synuclein contributes to neurotoxicity in the brain? still remain to be answered. A basic fundamental understanding of the key players in such a complex problem is extremely important. Therefore we hope that the results presented in this thesis contribute to a better understanding of the protein $\alpha$-synuclein and how it might be involved in disease. 


\section{Samenvatting}

De ziekte van Parkinson is een neurodegeneratieve aandoening die veel voorkomt bij mensen op latere leeftijd. Het neuronale eiwit $\alpha$-synucleine speelt waarschijnlijk een belangrijke rol bij de oorzaak en de progressie van de ziekte. Het eiwit is de hoofdcomponent van Lewy-lichaampjes die het belangrijkste neuropathologische kenmerk van de ziekte zijn. Lewy-lichaampjes zijn intracellulaire inclusies die grote hoeveelheden geaggregeerd (samengeklonterd) eiwit bevatten. Daarnaast zijn er mutaties en multiplicaties in het gen wat codeert voor $\alpha$-synucleine, die een erfelijke vorm van de ziekte van Parkinson veroorzaken. Een van de belangrijkste vragen is hoe de aggregatie van $\alpha$-synucleine te maken heeft met de ziekte. Recentelijk is men tot het idee gekomen dat een oligomere vorm van het eiwit, welke voorkomt als een tussen-stadium in het aggregatie proces, giftig zou kunnen zijn voor neuronen. Een mogelijk mechanisme waardoor deze oligomeren schadelijk kunnen zijn, is door het lek maken van membranen in de cel. In dit proefschrift proberen we de interactie van oligomeer $\alpha$-synucleine met lipide membranen te karakteriseren en te begrijpen.

Een stabiele oligomere vorm van $\alpha$-synucleine kan in vitro geprepareerd worden. Deze oligomeren bevatten ongeveer 40 tot 80 monomeren. Hoewel de oligomeren qua secundaire structuur $\beta$-sheet rijk zijn, net als de grote fibrillaire aggregaten die in de hersenen voorkomen, hebben de oligomeren niet dezelfde structuur als deze fibrillen. De oligomeren liggen waarschijnlijk niet op de route van de formatie van fibrillen uit monomeer eiwit. De aggregatie van monomeer $\alpha$-synucleine kon niet geïnduceerd worden door toevoeging van een kleine hoeveelheid oligomeren (Hoofdstuk 2).

De oligomere vorm van $\alpha$-synucleine kan lipide membranen verstoren. Als deze oligomeren worden toegevoegd aan kleine synthetische vesikels, dan zullen de oligomeren het membraan lek maken. De permeabilisatie van het membraan is voornamelijk afhankelijk van de lading van het membraan. Alleen vesikels waarvan het membraan bestaat uit negatief geladen lipiden, worden door het eiwit verstoord. Daarnaast is ook de pakking van de lipiden in het membraan van belang (Hoofdstuk 3).

Het feit dat alleen negatief geladen membranen verstoord worden, wordt voornamelijk veroorzaakt door de bindingseigenschappen van de oligomeren. Oligomeer $\alpha$-synucleine bindt alleen aan het membraan als er voldoende negatief geladen lipiden aanwezig zijn . De binding van oligomeren aan negatief geladen vesikels heeft een dissociatie constante in de orde van grootte van de micro- 
molair. De affiniteit voor het membraan neemt af bij afnemende lading van het membraan (Hoofdstuk 4 en 5). Deze bindingseigenschappen impliceren dat het oligomeer bepaalde structuur elementen moet hebben zoals positief geladen gedeeltes of een bepaalde flexibiliteit.

De bindingseigenschappen van de oligomeren zijn een gevolg van hun structuur. De hoge affiniteit voor negatief geladen lipiden suggereert dat de positief geladen residuen in the N-terminus van het eiwit, een rol spelen in het bindingsproces. Door mutanten te maken met een tryptofaan residu op verschillende locaties in het eiwit, kan men wat over de chemische omgeving zeggen waarin dit deel van het eiwit zich bevindt. De resultaten van tryptofaan fluorescentie spectroscopie laten zien dat de N-terminus verantwoordelijk is voor de membraanbinding van oligomeer $\alpha$-synucleine (Hoofdstuk 6). Daarnaast is de C-terminus van het eiwit blootgesteld aan water en wordt de kern van het aggregaat gevormd door de N-terminus.

Hoe membraan gebonden oligomeren de lipide bilaag lek maken is nog steeds niet duidelijk. Een model waarbij het oligomeer een complex vormt in het membraan, dat lijkt op porie-vormende eiwitten, is op dit moment het meest populair in de literatuur. Onze resultaten kunnen dit model niet weerleggen. Uit de experimenten blijkt echter, dat defecten in het membraan, een zeer grote rol kunnen spelen in het lek raken van het membraan. Vesikels die gemaakt worden uit enkel negatief geladen lipiden, zijn intrinsiek instabiel. Op het moment dat er eiwitten aan het oppervlakte binden, kunnen er zeer grote defecten ontstaan, die een groot deel van de membraan permeabiliteit verklaren. Zelfs onder omstandigheden dat het membraan gestabiliseerd wordt, zien wij aanwijzingen voor defecten (Hoofdstuk 7).

Hoewel we de membraan interactie van oligomeer $\alpha$-synucleine steeds beter begrijpen, is niet duidelijk welke rol dit proces speelt in de ziekte van Parkinson. Naast de hypothese waar wij aan werken, zijn er meerdere veelbelovende ideeën over wat er gebeurt in het brein tijdens de ziekte. Het is extreem complex om vast te stellen hoe de ziekte van Parkinson werkt en wat de onderliggende mechanismen zijn. Onderzoek naar de rol van eiwit aggregatie in neurodegeneratieve ziekten is al minstens tien jaar aan de gang. Echter, zelfs fundamentele vragen als: Heeft het aggregatieproces van dit soort eiwitten nadelige gevolgen voor het brein? zijn nog steeds niet beantwoord. Een fundamenteel begrip van de componenten die een rol spelen in dit soort ziekten is belangrijk. We hopen dat de resultaten in dit proefschrift, leiden tot een beter begrip van het eiwit $\alpha$-synucleine en welke rol het eiwit speelt in de ziekte van Parkinson. 


\section{Dankwoord}

Graag zou ik iedereen willen bedanken die heeft bijgedragen aan de totstandkoming van dit proefschrift.

Vinod, bedankt voor het ongelimiteerde vertrouwen en de vrijheid die ik gedurende mijn promotie gekregen heb. Je enthousiasme, wetenschappelijke kennis en het vermogen om de juiste randvoorwaarden voor onderzoek te scheppen zijn bewonderenswaardig. Daarnaast heb je me altijd gestimuleerd om groot te denken en nieuwe projecten aan te pakken.

Mireille, ik denk dat je precies op het juiste moment bij ons in de groep bent gekomen. Zonder jou was mijn project zeker in die fase minder soepel verlopen. Ook nam je altijd de tijd om mijn nieuwste data te bespreken. Hoewel we na deze discussies vaak meer vragen hadden dan waar we ooit mee begonnen, heb ik hier altijd veel plezier aan beleefd.

Ik heb tijdens mijn onderzoek de luxe gehad om op elk willekeurig moment de vriezer te kunnen openen en hier de gewenste hoeveelheid eiwit uit te halen die ik nodig had voor mijn experimenten. Dit is te danken aan de inzet van Kirsten, Marloes en Yvonne. Daarnaast heeft Kirsten het grootste deel van de tryptofaan metingen uitgevoerd. Bedankt voor jullie grote bijdrage aan mijn onderzoek en voor de goede samenwerking in het lab.

Ook heb ik het geluk gehad dat er altijd wel mensen rondliepen die veel meer wisten van eiwitten dan ik. Rolf je bijdrage in het begin van mijn project was zeer belangrijk, een goed begin is het halve werk! Gertjan bedankt voor de fijne samenwerking, je kennis en ervaring hebben een grote bijdrage geleverd aan mijn werk en aan de synucleine groep in het algemeen. Ine, je was altijd bereid om te helpen in het lab, ook stond jouw deur altijd open als ik even wat te klagen had over problemen die promovendi nou eenmaal hebben.

Daarnaast zijn er gedurende mijn promotie nog vele anderen geweest, die een bijdrage geleverd hebben aan dit proefschrift. Ik zal proberen niemand te vergeten en een opsomming maken in min of meer chronologische volgorde. De volgende mensen wil ik graag bedanken: Maryana, we started on the exact same day, thanks for all your help and good luck with your defense! Kees voor je hulp met de AFM. Ik weet dat je het leuk had gevonden als ik mijn promotie hier meer op gericht had. Yanina for taking a lot of time in helping me out with the bilayer experiments! Wei Zhou from the PBM group for helping with the DLS experiments. Enrico Marani en Remi Wiertz uit de BSS groep, voor hun inzet bij de de initiële toxiciteits studies op neuronale cellen. Malte and Martina, for 
our wonderful and fruitful collaboration over the past years. Sachin Kinge from the SMCT for his help with the CD experiments. Mark Smithers van MESA+ voor de TEM plaatjes van de oligomeren. Prof. Dr. Mathias Winterhalter and Mahendran from the Jacobs university in Bremen, a place for great science and even better coffee, thanks for having me as a guest and facilitating the single channel recordings. Tom voor zijn hulp met de toxiciteits studie op de neuroblastoma cellen. Aufried, Dianwen en Cees voor hun bijdrage aan de FCS metingen. Frans en Sylvia voor hun hulp in allerlei praktische zaken. All BPE members for being great colleagues. And finally everyone participating in the synuclein group-meeting, for the useful discussions, suggestions and help I have received over the past years. 


\section{List of publications}

\section{About the author}

Bart van Rooijen was born on March 8th, 1981 in Warmenhuizen, The Netherlands. After graduating from high school in Alkmaar in 1999, he studied Applied Physics at the University of Twente in Enschede, The Netherlands. After obtaining his M.Sc. in Applied Physics in 2005, he joined the Biophysical Engineering Group as a Ph.D. candidate. The project, supervised by Mireille Claessens and Vinod Subramaniam, resulted in this thesis.

\section{List of publications}

\section{Published papers}

van Rooijen, B.D., van Leijenhorst-Groener, K.A., Claessens, M.M.A.E. and Subramaniam, V. (2009) Tryptophan fluorescence reveals structural features of $\alpha$-synuclein oligomers. J. Mol. Biol. doi:10.1016/j.jmb.2009.10.021, manuscript accepted.

van Rooijen, B.D., Claessens, M.M.A.E., and Subramaniam, V. (2009) Lipid bilayer disruption by oligomeric alpha-synuclein depends on bilayer charge and accessibility of the hydrophobic core 2009. Biochim. Biophys. Acta - Biomembr. 1788(6), 1271-1278.

van Rooijen, B.D., Claessens, M.M.A.E. and Subramaniam, V. (2008) Membrane binding of oligomeric alpha-synuclein depends on bilayer charge and packing. FEBS Lett. 528(27), 3788-3792.

Drescher, M., Godschalk, F., Veldhuis, G., van Rooijen, B.D., Subramaniam, V., and Huber, M. (2008) Spin-label EPR on alpha-synuclein reveals differences in the membrane binding affinity of the two antiparallel helices. Chembiochem 9(15), 2411-2416.

Drescher, M., Veldhuis, G., van Rooijen, B.D., Milikisyants, S., Subramaniam, V. and Huber, .M (2008) Antiparallel arrangement of the helices of vesicle-bound alpha-synuclein. J. Am. Chem. Soc. 130(25), 7796.

Segers-Nolten, I.M., Wilhelmus, M.M., Veldhuis, G., van Rooijen, B.D., Drukarch, B. and Subramaniam, V. (2008) Tissue transglutaminase modulates alpha-synuclein oligomerization. Protein Sci. 17(8), 1395-1402. 


\section{Manuscripts in preparation}

Drescher, M., van Rooijen, B. D., Veldhuis, G., Subramaniam, V., and Huber, M. A stable lipid-induced aggregate of alpha-synuclein. Manuscript submitted.

van Rooijen, B.D., Otto, C., Claessens, M. M. A. E. and Subramaniam V. Quantitative fluorescence correlation spectroscopy of membrane binding by oligomeric $\alpha$-synuclein reveals the importance of charge interactions. Manuscript submitted.

van Rooijen, B.D., Claessens, M. M. A. E., Claessens and Subramaniam, V. Membrane permeabilization by oligomeric $\alpha$-synuclein: In search of the mechanism. Manuscript in preparation.

van Rooijen, B. D., Claessens, M. M. A. E. and Subramaniam, V. The , The interaction of oligomeric $\alpha$-synuclein with lipid membranes. Invited review, Manuscript in preparation. 

